\title{
Subcritical transition in channel flows
}

\author{
By S. JONATHAN CHAPMAN \\ OCIAM, Mathematical Institute, 24-29 St. Giles', Oxford OX1 3LB, UK
}

(Received 17 August 1999 and in revised form 28 June 2001)

Certain laminar flows are known to be linearly stable at all Reynolds numbers, $R$, although in practice they always become turbulent for sufficiently large $R$. Other flows typically become turbulent well before the critical Reynolds number of linear instability. One resolution of these paradoxes is that the domain of attraction for the laminar state shrinks for large $R$ (as $R^{\gamma}$ say, with $\gamma<0$ ), so that small but finite perturbations lead to transition. Trefethen et al. (1993) conjectured that in fact $\gamma<-1$. Subsequent numerical experiments by Lundbladh, Henningson \& Reddy (1994) indicated that for streamwise initial perturbations $\gamma=-1$ and $-7 / 4$ for plane Couette and plane Poiseuille flow respectively (using subcritical Reynolds numbers for plane Poiseuille flow), while for oblique initial perturbations $\gamma=-5 / 4$ and $-7 / 4$. Here, through a formal asymptotic analysis of the Navier-Stokes equations, it is found that for streamwise initial perturbations $\gamma=-1$ and $-3 / 2$ for plane Couette and plane Poiseuille flow respectively (factoring out the unstable modes for plane Poiseuille flow), while for oblique initial perturbations $\gamma=-1$ and $-5 / 4$. Furthermore it is shown why the numerically determined threshold exponents are not the true asymptotic values.

\section{Introduction}

It is well known that certain laminar flows are linearly stable (i.e. stable to infinitesimal perturbations) at all Reynolds numbers, $R$, but in practice always become turbulent for sufficiently large Reynolds number. Examples include plane Couette flow and pipe Poiseuille flow (Romanov 1973; Davey 1973; Drazin \& Reid 1981). On the other hand, plane Poiseuille flow does exhibit a critical Reynolds number $R_{c} \approx 5772$ (Orszag 1971) at which an unstable mode appears (the so-called Tollmien-Schlichting wave), but in practice the transition for this flow also often occurs at much lower Reynolds numbers. The resolution of these paradoxes is a long-standing problem in fluid mechanics. One school of thought is that the domain of attraction of the laminar flow shrinks as $R \rightarrow \infty$, so that this flow is nonlinearly unstable to small but finite perturbations. This idea goes back at least to Thomson (1887) and Orr (1907).

Almost all of the work on transition in plane Poiseuille flow is concerned with the neutral curve (see, for example Drazin \& Reid 1981). One theory of subcritical transition is based on a weakly nonlinear analysis about this curve, as in Stuart (1960) and Watson (1960) (see also Reynolds \& Potter 1967). The Landau equation for the amplitude of the linearly neutral solution shows that the bifurcation is subcritical, and the position of the steady (periodic) state determines the threshold amplitude for transition. A similar approach has been attempted for plane Couette flow, in which the 'bifurcation' to a non-trivial state occurs at $R=\infty$ rather than a finite value of $R$. Cowley \& Smith (1985), Nagata (1990), Ehrenstein \& Koch (1991) and Cherhabili $\&$ Ehrenstein (1997) all look for nonlinear steady or periodic states which are close to the laminar state, and which are seen as determining the threshold amplitude for 
transition. However, while such steady states certainly give an upper bound on the threshold amplitude for transition, the threshold itself is determined by the stable manifold through these states, which is an order of magnitude harder to determine (even if we could find the 'closest' non-trivial solution). This point is illustrated well in the toy model of $\S 2$ : even though the number of degrees of freedom is only increased from one (the amplitude in the Landau equation) to two, the scaling of the threshold amplitude differs from that of the nearest non-trivial steady state.

An alternative approach is to look directly at the initial value problem. Based on the observation that the route to transition is invariably three-dimensional (even in the case of plane Poiseuille flow, for which the one unstable mode is two-dimensional), many theories look for a secondary instability of a solution of the linearized equations. Weakly nonlinear theories have been proposed by Benney \& Lin (1960) and Benney (1961, 1964), in which two terms of an amplitude expansion are kept, with the first term slowly decaying. The second-order term is observed to perturb the mean flow towards an inflectional profile, which would then generate a secondary instability. No attempt is made to relate the amplitude expansion to the Reynolds number and thereby obtain a threshold amplitude, so that the approach remains slightly ad hoc; the second-order terms can never perturb the mean flow enough enough to produce instability in the asymptotic limit considered. Nevertheless, these works were among the first to realise the importance of streamwise vorticity in redistributing momentum, a mechanism which is a key ingredient of the present analysis, and which we will return to shortly.

The obvious solution of the linearized equations to examine in this fashion is the most slowly decaying mode, which gives the greatest time for instability to set in. Orszag \& Kells (1980) and Orszag \& Patera (1983) examine numerically the secondary instability of the least stable (and therefore two-dimensional) mode for plane Couette and subcritical plane Poiseuille flow. They find that finite-amplitude two-dimensional disturbances can become catastrophically unstable to three-dimensional perturbations. Davey \& Nguyen (1971) attempt to obtain a threshold amplitude in this way using an ad hoc truncation of a nonlinear perturbation of the least stable mode.

However, it was later realised that there may be solutions of the linearized equations which actually grow transiently in time before they finally decay, and that a secondary instability of these solutions is more likely to be the optimal route to transition. Early work (Gustavsson \& Hultgren 1980; Benney \& Gustavsson 1981; Gustavsson 1986; Jang, Benney \& Gran 1986; Shantini 1989) looked for an exact resonance of an Orr-Sommerfeld and a Squire eigenvalue to achieve this transient growth.

Later it was realised that in fact exact resonance is not necessary to obtain transient growth. The fact that the eigenfunctions of the linearized Navier-Stokes equations are not orthogonal (i.e. the operator is non-normal) is enough to give the existence of initial conditions which exhibit transient growth before finally decaying (Farrell 1988; Gustavsson 1991; Henningson 1991; Butler \& Farrell 1992; Henningson, Lundbladh \& Johansson 1993), and in fact there is no additional growth at an exact resonance (Reddy \& Henningson 1993). For large Reynolds number the operator is highly non-normal, and the transient growth is asymptotically large in $R$. In Reddy \& Henningson (1993) and Reddy, Schmid \& Henningson (1993) the non-normality of the linearized operator is examined using pseudospectra, and linked to the transient growth.

The idea that the small domain of attraction is caused by a 'linear amplification plus nonlinear mixing' (Boberg \& Brosa 1988) has been built on by Trefethen et al. (1993), who propose simple toy models in which the conservative nonlinear terms feed 
the transient linear growth back into the system, leading to a low threshold amplitude for transition. Various other low-dimensional models with these qualitative features are reviewed in Baggett \& Trefethen (1997).

A key question, posed in Trefethen et al. (1993), is to determine the behaviour of the threshold amplitude for transition as a function of $R$ as $R \rightarrow \infty$, that is, to determine the smallest $\gamma$ such that a perturbation of norm $O\left(R^{\gamma}\right)$ may lead to transition. While this question makes sense as it stands for plane Couette flow, which is linearly stable for all Reynolds numbers, it requires some interpretation for plane Poiseuille flow, for which an infinitesimal perturbation will lead to transition as $R \rightarrow \infty$, since the flow is linearly unstable. In that case we will factor out the unstable mode and consider the threshold amplitude of the remaining linearly stable system. The reason such an analysis is interesting is that while the asymptotic analysis to follow will be based on $R \rightarrow \infty$, we would expect that the results are qualitatively similar in the region $R<5772$ (since $5772 \gg 1$ ), in which the flow is linearly stable. We expect the small domain of attraction of the laminar state and the route to transition at subcritical Reynolds numbers to be qualitatively similar to that of the stable part of the system as $R \rightarrow \infty$. In fact, such an interpretation is not necessary for the periodic flows with period independent of Reynolds number with which we will be primarily concerned, since for them plane Poiseuille flow is linearly stable in the limit as $R \rightarrow \infty$, because the unstable streamwise wavenumbers $\alpha$ on an infinite domain lie in a band $O\left(R^{-1 / 7}\right) \leqslant \alpha \leqslant O\left(R^{-1 / 11}\right)$ (see, for example, Drazin \& Reid 1981).

It was conjectured in Trefethen et al. (1993), based on their simple models, that $\gamma<-1$, which appears to be the first conjecture on the value of the exponent. Kreiss, Lundbladh \& Henningson (1994) prove a lower bound for $\gamma$ for plane Couette flow of $-21 / 4$, while numerical experiments by Lundbladh, Henningson \& Reddy (1994) and Reddy et al. (1998) suggest that $\gamma \approx-5 / 4$ for plane Couette flow and $\gamma \approx-7 / 4$ for plane Poiseuille flow in a periodic domain.

In fact there are two routes to transition examined in Lundbladh et al. (1994) and Reddy et al. (1998), which are

(i) streamwise vortices $\rightarrow$ streamwise streaks

$\rightarrow$ secondary instability of oblique modes,

(ii) oblique modes $\rightarrow$ streamwise vortices $\rightarrow$ streamwise streaks

$\rightarrow$ secondary instability of oblique modes.

A streamwise vortex is an elongated region of vorticity approximately aligned with the basic laminar flow, while a streamwise streak is an elongated region of relatively high or low velocity approximately aligned with this flow. By oblique mode we mean any perturbation not aligned with the flow, that is, a perturbation which is streamwise dependent. In (i) the non-normality of the linearized equations means that a small streamwise vortex produces a large streamwise streak as it decays, which then produces a secondary oblique instability. In (ii) an initial perturbation in the form of a pair of oblique modes grows transiently at the same time generating a streamwise vortex. The route to transition then proceeds as in (i).

We will be concerned here with channel flows, namely plane Couette and plane Poiseuille flow. We will determine the scaling of the threshold amplitude for the routes (i) and (ii) to transition by using an asymptotic analysis of the Navier-Stokes equations to quantify the qualitative ideas of Trefethen et al. (1993). We will also determine the threshold amplitude for the alternative route to transition

(iii) streamwise vortices $\rightarrow$ streamwise streaks $\rightarrow$ streamwise vortices, 


\begin{tabular}{|c|c|c|c|c|c|}
\hline \multicolumn{3}{|c|}{ Numerical experiments } & \multicolumn{3}{|c|}{ Asymptotic analysis } \\
\hline & Couette & Poiseuille & & Couette & Poiseuille \\
\hline $\begin{array}{l}\text { Streamwise } \\
\text { Oblique }\end{array}$ & $\begin{array}{l}-1 \\
-5 / 4\end{array}$ & $\begin{array}{l}-7 / 4 \\
-7 / 4\end{array}$ & $\begin{array}{l}\text { Streamwise } \\
\text { Oblique }\end{array}$ & $\begin{array}{l}-1 \\
-1\end{array}$ & $\begin{array}{l}-3 / 2 \\
-5 / 4\end{array}$ \\
\hline
\end{tabular}

TABLE 1. Numerically and asymptotically determined threshold exponents for initial perturbations in the form of optimally growing streamwise vortices and optimally growing oblique modes in plane Couette and plane Poiseuille flow with periodic boundary conditions.

in which the streamwise streaks generate streamwise vortices, closing the loop rather than generating a secondary instability. We will find that the lowest threshold exponent for plane Couette flow is $\gamma=-1$, which occurs for routes (i), (ii) and (iii), while that for plane Poiseuille flow occurs via route (i), and is $\gamma=-3 / 2$. We will also explain why the numerical computations mentioned above obtained different exponents (see table 1).

We begin by introducing a low-dimensional toy model which illustrates the routes to transition of the Navier-Stokes equations described above, and with which we are able to explain the main ideas involved. Then, in $\S 3$ we formulate the Navier-Stokes equations in a convenient way for the subsequent analysis. In $\S 4$ we determine the nature and magnitude of the transient growth in the (stable) linearized equations. In $\S 5$ we show how to calculate the asymptotic position of the eigenvalues of the linearized equations, which will enable us to determine how large a perturbation of the base flow needs to be in order to significantly perturb these eigenvalues. In $\S 6$ we briefly consider the nature of the secondary instability in the oblique modes before combining the results of $\S \S 4$ and 5 with an examination of the nonlinear equations in $\S 7$, which allows us to determine the threshold amplitude exponents for transition. In $\S 8$ we compare our asymptotic results to the numerical results of Lundbladh et al. (1994), Kreiss et al. (1994), Reddy et al. (1998). Finally, in $\S 9$, we present our conclusions.

\section{Some simple toy models}

We begin by illustrating the ideas involved with some simple toy models. Consider first the two-dimensional system introduced by Trefethen et al. (1993)

$$
\begin{gathered}
\frac{\mathrm{d} \psi}{\mathrm{d} t}+2 \epsilon \psi=\left(\phi^{2}+\psi^{2}\right)^{1 / 2} \phi, \\
\frac{\mathrm{d} \phi}{\mathrm{d} t}+\epsilon \phi-\psi=-\left(\phi^{2}+\psi^{2}\right)^{1 / 2} \psi .
\end{gathered}
$$

The variables $\psi$ and $\phi$ represent the input and output of a non-modal amplifier; for our present purposes we may think of them as representing streamwise vortices and streamwise streaks respectively in the route to transition (iii) above, though Trefethen et al. (1993) are deliberately not so specific. The nonlinear terms represent a conservative feedback mechanism.

Let us first consider the linearized equations. The linear system is stable but highly non-normal in that the two eigenvectors are almost parallel. This non-normality leads to the existence of initial conditions for which there is a large transient growth before the final decay. An initial condition of the form $(\phi, \psi)=(0, \epsilon \Psi)$ will generate a 


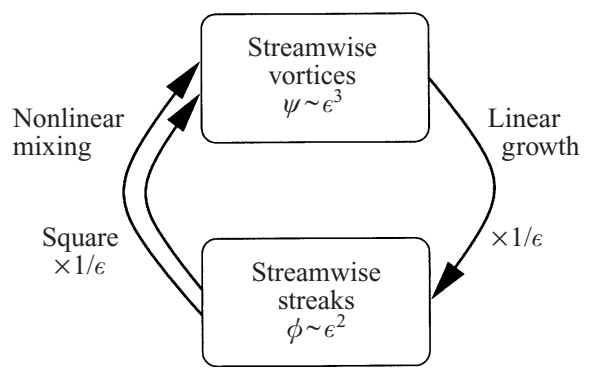

FIGURE 1. Schematic diagram of the route to transition (iii).

transient over a timescale of order $\epsilon^{-1}$ in which $\phi$ is order one. The factor of 2 is inserted into equation (2.1) so that the eigenvalues are distinct. In fact nothing special happens if the eigenvalues are repeated; the scalings and transient growth are the same. With distinct eigenvalues the transient growth is of the form $\mathrm{e}^{-\epsilon t}-e^{-2 \epsilon t}$, which becomes $\epsilon t \mathrm{e}^{-\epsilon t}$ if the factor of 2 is replaced by unity. A similar phenomenon occurs with the linearized Navier-Stokes equations: the non-normality determines the degree of transient growth, and the resonance or not of eigenvalues is unimportant.

Based on the observation above, we rescale the equations so that $\psi \sim \epsilon \phi$, and we rescale time so that we are working on the timescale for transient growth. The scale for $\phi$ is then chosen so that the largest nonlinear term balances with the linear part of the system. Thus we set

$$
t=\epsilon^{-1} \hat{t}, \quad \phi=\epsilon^{2} \hat{\phi}_{1}, \quad \psi=\epsilon^{3} \hat{\psi}_{1} .
$$

Keeping only the leading-order nonlinear terms in the new scaling the system becomes

$$
\begin{aligned}
& \frac{\mathrm{d} \hat{\psi}}{\mathrm{d} \hat{t}}+2 \hat{\psi}=\hat{\phi}|\hat{\phi}|, \\
& \frac{\mathrm{d} \hat{\phi}}{\mathrm{d} \hat{t}}+\hat{\phi}-\hat{\psi}=0 .
\end{aligned}
$$

Now, for initial conditions $(\hat{\phi}, \hat{\psi})=(0, \hat{\Psi})$ equation (2.5) generates a linear growth in $\hat{\phi}$, as $\hat{\psi}$ decays, which couples back nonlinearly into $\hat{\psi}$ through equation (2.4). This mechanism is illustrated schematically in figure 1, in which a single arrow represents a linear mechanism, while a double arrow represents a nonlinear mechanism. If $\hat{\Psi}$ is sufficiently large the solution will not converge to the origin (representing the laminar state) but to another stationary point (representing transition). Thus, from (2.3), the threshold amplitude for transition in this model is $O\left(\epsilon^{3}\right)$.

This simple model also serves to illustrate the point made in the introduction about the relationship between non-trivial steady states and threshold amplitudes. The existence of a threshold amplitude in (2.4)-(2.5) is due to the non-trivial stationary points $\hat{\phi}=\hat{\psi}= \pm 2$. However, knowing that this solution exists is not enough to determine the threshold amplitude, since the threshold is determined by the stable manifold through this stationary point. In particular, this stationary point lies a distance $O\left(\epsilon^{2}\right)$ from the origin (the scaling on $\phi$ ), while the threshold amplitude is $O\left(\epsilon^{3}\right)$ (since an initial perturbation with $\hat{\phi}=0$ and $\hat{\psi}$ sufficiently large will lead to transition).

Although this toy model illustrates many of the features of transition through route (iii), we will find when we consider the Navier-Stokes equations in $\S 4$ that 
the nonlinear coupling between streamwise streaks and streamwise vortices is much weaker than that given by (2.1)-(2.2); in fact the nonlinear terms are multiplied by a factor of $\epsilon^{2}$, so that the threshold amplitude for transition is only $O(\epsilon)$.

It turns out that the dominant routes to transition are (i) and (ii). A simple toy model which illustrates these routes to transition is the four-dimensional system

$$
\begin{gathered}
\frac{\mathrm{d} \psi_{1}}{\mathrm{~d} t}+2 \epsilon \psi_{1}=\phi_{2}^{2}, \\
\frac{\mathrm{d} \phi_{1}}{\mathrm{~d} t}+\epsilon \phi_{1}-\psi_{1}=0, \\
\frac{\mathrm{d} \psi_{2}}{\mathrm{~d} t}+2 \delta \psi_{2}=\phi_{1} \phi_{2}, \\
\frac{\mathrm{d} \phi_{2}}{\mathrm{~d} t}+\delta \phi_{2}-\psi_{2}=0 .
\end{gathered}
$$

Here $\phi_{1}$ and $\psi_{1}$ represent streamwise streaks and streamwise vortices respectively, while $\phi_{2}$ and $\psi_{2}$ represent all the oblique modes.

The linearized equations decouple into a pair of stable, non-normal two-dimensional systems of the form (2.1)-(2.2); the first has transient growth of order $\epsilon^{-1}$ over a timescale $\epsilon^{-1}$, while the second has transient growth of order $\delta^{-1}$ over a timescale $\delta^{-1}$. We assume that $0<\epsilon \ll \delta \ll 1$; we will find that this is the case for the Navier-Stokes equations.

The nonlinear terms have been simplified (based on the observation above that the important feedback is through $\phi$ ), and are chosen to be consistent with the coupling between streamwise and oblique modes: the product of two non-zero-wavenumber (oblique) modes may give a zero-wavenumber (streamwise) mode, while the product of a zero-wavenumber mode and a non-zero-wavenumber mode gives a non-zerowavenumber mode.

We rescale the equations so that $\psi_{1} \sim \epsilon \phi_{1}$ and $\psi_{2} \sim \delta \phi_{2}$. The scales for $\phi_{1}$ and $\phi_{2}$ are chosen so that the nonlinear terms balance with the linear part of each twodimensional system. We also scale time so that we are working on the shorter of the two timescales. Thus we set

$$
t=\delta^{-1} \hat{t}, \quad \phi_{1}=\delta^{2} \hat{\phi}_{1}, \quad \psi_{1}=\epsilon \delta^{2} \hat{\psi}_{1}, \quad \phi_{2}=\epsilon \delta \hat{\phi}_{2}, \quad \psi_{2}=\epsilon \delta^{2} \hat{\psi}_{2} .
$$

In the new scaling the system becomes

$$
\begin{gathered}
\left(\frac{\delta}{\epsilon}\right) \frac{\mathrm{d} \hat{\psi}_{1}}{\mathrm{~d} \hat{t}}+2 \hat{\psi}_{1}=\hat{\phi}_{2}^{2}, \\
\left(\frac{\delta}{\epsilon}\right) \frac{\mathrm{d} \hat{\phi}_{1}}{\mathrm{~d} \hat{t}}+\hat{\phi}_{1}-\hat{\psi}_{1}=0, \\
\frac{\mathrm{d} \hat{\psi}_{2}}{\mathrm{~d} \hat{t}}+2 \hat{\psi}_{2}=\hat{\phi}_{1} \hat{\phi}_{2}, \\
\frac{\mathrm{d} \hat{\phi}_{2}}{\mathrm{~d} \hat{t}}+\hat{\phi}_{2}-\hat{\psi}_{2}=0 .
\end{gathered}
$$

Now, on this short timescale we see from (2.11), (2.12) that to leading order $\mathrm{d} \hat{\phi}_{1} / \mathrm{d} \hat{t}=$ $\mathrm{d} \hat{\psi}_{1} / \mathrm{d} \hat{t}=0$, so that $\hat{\phi}_{1}$ and $\hat{\psi}_{1}$ are constant. The system for the remaining variables 
is then linear, with eigenvalues given by

$$
\lambda=-1 \pm\left(\hat{\phi}_{1}\right)^{1 / 2} \text {. }
$$

For $\operatorname{Re}\left(\left(\hat{\phi}_{1}\right)^{1 / 2}\right)>1$ one eigenvalue has positive real part, corresponding to an oblique mode instability. For $\operatorname{Re}\left(\left(\hat{\phi}_{1}\right)^{1 / 2}\right)<1$ both eigenvalues have negative real part, and the oblique modes decay to zero. Let us now determine the scaling of the threshold amplitude for initial conditions in the form of streamwise vortices and in the form of oblique modes.

We consider first the route to transition (i), which requires an initial perturbation in the form of streamwise vortices, corresponding to an initial condition of the form $\left(\hat{\phi}_{1}, \hat{\psi}_{1}, \hat{\phi}_{2}, \hat{\psi}_{2}\right)=\left(0, \hat{\Psi}_{1}, 0,0\right)$. Since $\hat{\phi}_{1}$ and $\hat{\psi}_{1}$ evolve over the long timescale $\hat{t} \sim \delta / \epsilon$, we first rescale time with $\delta / \epsilon$ to give

$$
\begin{gathered}
\frac{\mathrm{d} \hat{\psi}_{1}}{\mathrm{~d} \tilde{t}}+2 \hat{\psi}_{1}=\hat{\phi}_{2}^{2}, \\
\frac{\mathrm{d} \hat{\phi}_{1}}{\mathrm{~d} \tilde{t}}+\hat{\phi}_{1}-\hat{\psi}_{1}=0, \\
\left(\frac{\epsilon}{\delta}\right) \frac{\mathrm{d} \hat{\psi}_{2}}{\mathrm{~d} \tilde{t}}+2 \hat{\psi}_{2}=\hat{\phi}_{1} \hat{\phi}_{2}, \\
\left(\frac{\epsilon}{\delta}\right) \frac{\mathrm{d} \hat{\phi}_{2}}{\mathrm{~d} \tilde{t}}+\hat{\phi}_{2}-\hat{\psi}_{2}=0,
\end{gathered}
$$

where $\hat{t}=(\delta / \epsilon) \tilde{t}$. Since initially $\operatorname{Re}\left(\left(\hat{\phi}_{1}\right)^{1 / 2}\right)<1$ if there were any oblique modes they would decay quickly to zero. Thus we are left with the stable linear system

$$
\begin{gathered}
\frac{\mathrm{d} \hat{\psi}_{1}}{\mathrm{~d} \tilde{t}}+2 \hat{\psi}_{1}=0, \\
\frac{\mathrm{d} \hat{\phi}_{1}}{\mathrm{~d} \tilde{t}}+\hat{\phi}_{1}-\hat{\psi}_{1}=0,
\end{gathered}
$$

for $\hat{\phi}_{1}$ and $\hat{\psi}_{1}$. Now our initial condition of the form $\left(\hat{\phi}_{1}, \hat{\psi}_{1}\right)=\left(0, \hat{\Psi}_{1}\right)$ will give transient growth in $\hat{\phi}_{1}$ while $\hat{\psi}_{1}$ decays to zero (streamwise vortices producing streamwise streaks). If this transient growth is enough to take $\operatorname{Re}\left(\left(\hat{\phi}_{1}\right)^{1 / 2}\right)$ above unity, then the system for $\hat{\phi}_{2}, \hat{\psi}_{2}$ becomes unstable on a short timescale. An infinitesimal perturbation of this system has exponential growth with a rate of order $\delta / \epsilon$ on the long timescale over which $\hat{\phi}_{1}$ and $\hat{\psi}_{1}$ are decaying. Such a perturbation will therefore quickly become order one, the nonlinear terms in (2.16) 'kick in' to keep $\hat{\phi}_{1}$ above unity, and the feedback leads to transition away from the zero state. Thus an order-one $\hat{\psi}_{1}$ is enough to induce transition, so that, by (2.10), the threshold amplitude for streamwise vortices is $O\left(\epsilon \delta^{2}\right)$. This route to transition is shown schematically in figure 2 .

Let us now consider the route to transition (ii), requiring an initial condition in the form of oblique modes, $\left(\hat{\phi}_{1}, \hat{\psi}_{1}, \hat{\phi}_{2}, \hat{\psi}_{2}\right)=\left(0,0,0, \hat{\Psi}_{2}\right)$. On the short timescale $\hat{t} \sim 1$ over which $\hat{\psi}_{2}$ decays there will be transient growth in $\hat{\phi}_{2}$. However, in the present scaling, during this transient growth phase the nonlinear terms in (2.11) generate a streamwise vortex $\hat{\psi}_{1}$ only of order $\epsilon / \delta$, whereas we have seen that a streamwise vortex $\hat{\psi}_{1}$ of order one is required for transition to occur. In order to generate a streamwise vortex of order one from an oblique mode, the nonlinear terms in (2.11) 


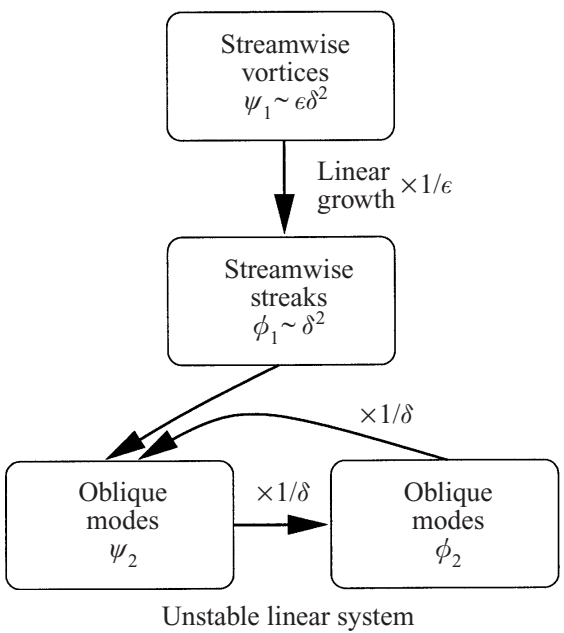

FIGURE 2. Schematic diagram of the route to transition (i).

must therefore of order $\delta / \epsilon$ so that they balance with $\mathrm{d} \hat{\psi}_{1} / \mathrm{d} \hat{t}$. Hence we must rescale

$$
\begin{gathered}
\phi_{2}=\epsilon \delta \hat{\phi}_{2}=\epsilon^{1 / 2} \delta^{3 / 2} \tilde{\phi}_{2}, \\
\psi_{2}=\epsilon \delta^{2} \hat{\psi}_{2}=\epsilon^{1 / 2} \delta^{5 / 2} \tilde{\psi}_{2},
\end{gathered}
$$

to give, on the short timescale

$$
\begin{gathered}
\frac{\mathrm{d} \hat{\psi}_{1}}{\mathrm{~d} \hat{t}}=\tilde{\phi}_{2}^{2}, \\
\frac{\mathrm{d} \hat{\phi}_{1}}{\mathrm{~d} \hat{t}}=0, \\
\frac{\mathrm{d} \tilde{\psi}_{2}}{\mathrm{~d} \hat{t}}+2 \tilde{\psi}_{2}=\hat{\phi}_{1} \tilde{\phi}_{2}, \\
\frac{\mathrm{d} \tilde{\phi}_{2}}{\mathrm{~d} \hat{t}}+\tilde{\phi}_{2}-\tilde{\psi}_{2}=0 .
\end{gathered}
$$

Now an initial perturbation in the form of an oblique mode will generate an orderone streamwise vortex as it decays, which will then generate an order-one streamwise streak and the route to transition proceeds as above. Hence the threshold amplitude for an initial perturbation comprising oblique modes is $O\left(\epsilon^{1 / 2} \delta^{5 / 2}\right)$. This route to transition is shown schematically in figure 3 .

Notice that during each phase of the transition (until the nonlinear evolution of the fully developed secondary oblique mode instability) we only have to solve linear equations. During the first phase of the transient growth of oblique modes we have the linear system (2.26)-(2.27) (remember that the right-hand side of (2.26) is zero during this phase). Then $\tilde{\phi}_{2}$ is known, so that (2.24) is a forced linear equation for $\hat{\psi}_{1}$. During the next phase we have the linear system (2.20)-(2.21) to describe the evolution of $\hat{\phi}_{1}$ and $\hat{\psi}_{1}$. Finally, the secondary oblique-mode instability is described by (2.13)-(2.14), which is a linear system for $\hat{\phi}_{2}, \hat{\psi}_{2}$, since $\hat{\phi}_{1}$ is known.

This scenario of transition, being governed by a series of (possibly forced) linear 


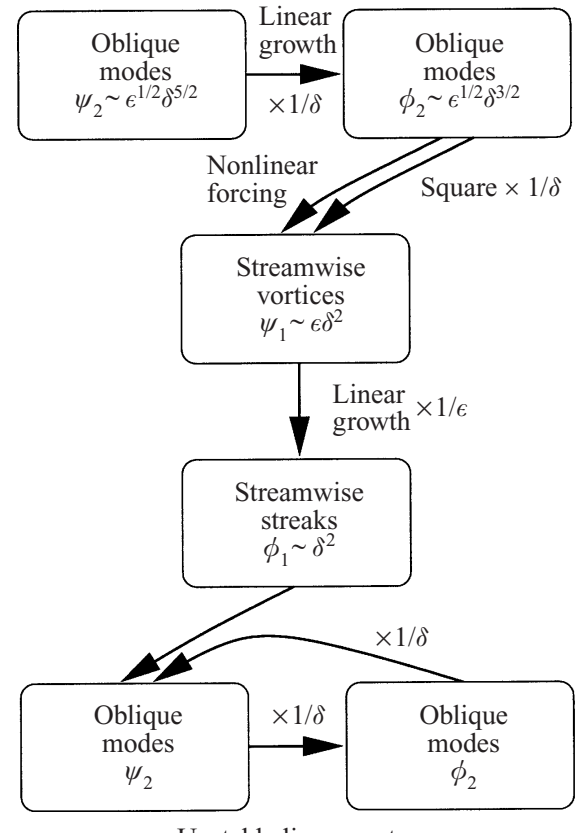

FIGURE 3. Schematic diagram of the route to transition (ii).

equations, persists when we consider the full Navier-Stokes equations, as do the routes to transition described above.

\section{Formulation of the equations}

Our starting point is of course the (dimensionless) incompressible Navier-Stokes equations

$$
\begin{gathered}
\boldsymbol{u}_{t}+\boldsymbol{u} \cdot \nabla \boldsymbol{u}=-\nabla p+\frac{1}{R} \nabla^{2} \boldsymbol{u}, \\
\nabla \cdot \boldsymbol{u}=0,
\end{gathered}
$$

with $\boldsymbol{u}$ given on $y= \pm 1$, where $\boldsymbol{u}$ is the fluid velocity, $p$ is the pressure, and $R$ is the Reynolds number. Taking the curl of (3.1) gives

$$
\omega_{t}+\operatorname{curl}(\omega \wedge \boldsymbol{u})=\frac{1}{R} \nabla^{2} \omega,
$$

where

$$
\omega=\operatorname{curl} \boldsymbol{u}
$$

is the vorticity. Let the laminar state whose stability we are examining be given by $\boldsymbol{u}=(U(y), 0,0)$ (we will be particularly interested in the cases $U=y$ corresponding to plane Couette flow and $U=1-y^{2}$ corresponding to plane Poiseuille flow, but in general $U$ may be any quadratic function of $y)$. We set $\boldsymbol{u}=(U, 0,0)+(u, v, w)$, $\omega=\left(0,0,-U^{\prime}\right)+(\xi, \eta, \zeta)$. Then the system (3.2)-(3.4) becomes

$$
\xi_{t}-(\eta U)_{y}+u_{z} U^{\prime}-\zeta_{z} U-\frac{1}{R}\left(\xi_{x x}+\xi_{y y}+\xi_{z z}\right)=-(\xi v-\eta u)_{y}+(-\xi w+\zeta u)_{z},
$$




$$
\begin{gathered}
\eta_{t}+\eta_{x} U+v_{z} U^{\prime}-\frac{1}{R}\left(\eta_{x x}+\eta_{y y}+\eta_{z z}\right)=(\xi v-\eta u)_{x}-(\eta w-\zeta v)_{z}, \\
\zeta_{t}-u_{x} U^{\prime}+\zeta_{x} U-\left(v U^{\prime}\right)_{y}-\frac{1}{R}\left(\zeta_{x x}+\zeta_{y y}+\zeta_{z z}\right)=-(-\xi w+\zeta u)_{x}+(\eta w-\zeta v)_{y}, \\
u_{x}+v_{y}+w_{z}=0, \\
\xi=w_{y}-v_{z}, \\
\eta=-w_{x}+u_{z}, \\
\zeta=v_{x}-u_{y},
\end{gathered}
$$

with $(u, v, w)=\mathbf{0}$ on $y= \pm 1$. Now, if we take the $z$-derivative of (3.5) and subtract the $x$-derivative of (3.7) we find

$$
\begin{aligned}
-\nabla^{2} v_{t}-U \nabla^{2} v_{x}+U^{\prime \prime} v_{x}+\frac{1}{R} \nabla^{4} v=- & (\xi v-\eta u)_{y z}+(-\xi w+\zeta u)_{z z} \\
& +(-\xi w+\zeta u)_{x x}-(\eta w-\zeta v)_{y x} .
\end{aligned}
$$

Equations (3.6) and (3.12) are convenient in that the linearized equations (Thomson 1887; Squire 1933)

$$
\begin{gathered}
-\nabla^{2} v_{t}-U \nabla^{2} v_{x}+U^{\prime \prime} v_{x}+\frac{1}{R} \nabla^{4} v=0 \\
\eta_{t}+\eta_{x} U+v_{z} U^{\prime}-\frac{1}{R} \nabla^{2} \eta=0
\end{gathered}
$$

involve only $v$ and $\eta$. If we decompose these into Fourier modes $\dagger$

etc., we find

$$
\begin{aligned}
& v=\sum v^{\alpha \beta}(y) \mathrm{e}^{\mathrm{i} \alpha x+\mathrm{i} \beta z}, \\
& \eta=\sum \eta^{\alpha \beta}(y) \mathrm{e}^{\mathrm{i} \alpha x+\mathrm{i} \beta z},
\end{aligned}
$$

where $k^{2}=\alpha^{2}+\beta^{2}$. Equations (3.8)-(3.11) give the Fourier components of the other variables as

$$
\begin{aligned}
u^{\alpha \beta} & =\frac{\mathrm{i}}{k^{2}}\left(\alpha v_{y}^{\alpha \beta}-\beta \eta^{\alpha \beta}\right), \\
w^{\alpha \beta} & =\frac{\mathrm{i}}{k^{2}}\left(\alpha \eta^{\alpha \beta}+\beta v_{y}^{\alpha \beta}\right), \\
\xi^{\alpha \beta} & =\frac{\mathrm{i}}{k^{2}}\left(\alpha \eta_{y}^{\alpha \beta}+\beta v_{y y}^{\alpha \beta}\right)-\mathrm{i} \beta v^{\alpha \beta}, \\
\zeta^{\alpha \beta} & =-\frac{\mathrm{i}}{k^{2}}\left(\alpha v_{y y}^{\alpha \beta}-\beta \eta_{y}^{\alpha \beta}\right)+\mathrm{i} \alpha v^{\alpha \beta} .
\end{aligned}
$$

$\dagger$ The description here assumes we are imposing periodic boundary conditions in $x$ and $z$. If we are instead working on an infinite domain, for Fourier series read Fourier transform, and for the sums in (3.23), (3.24) read integrals. 
We find that equations (3.12) and (3.6) become

$$
\begin{gathered}
L_{1}^{\alpha \beta}\left(v^{\alpha \beta}\right)=\sum_{\substack{\alpha_{1}+\alpha_{2}=\alpha \\
\beta_{1}+\beta_{2}=\beta}} N_{1}^{\alpha_{1} \beta_{1} \alpha_{2} \beta_{2}}\left(v^{\alpha_{1} \beta_{1}}, \eta^{\alpha_{1} \beta_{1}}, v^{\alpha_{2} \beta_{2}}, \eta^{\alpha_{2} \beta_{2}}\right), \\
L_{2}^{\alpha \beta}\left(\eta^{\alpha \beta}, v^{\alpha \beta}\right)=\sum_{\substack{\alpha_{1}+\alpha_{2}=\alpha \\
\beta_{1}+\beta_{2}=\beta}} N_{2}^{\alpha_{1} \beta_{1} \alpha_{2} \beta_{2}}\left(v^{\alpha_{1} \beta_{1}}, \eta^{\alpha_{1} \beta_{1}}, v^{\alpha_{2} \beta_{2}}, \eta^{\alpha_{2} \beta_{2}}\right),
\end{gathered}
$$

where the nonlinear terms on the right-hand side of (3.23) are of the form $v v_{y y y}, v_{y} v_{y y}$, $\eta v_{y y}, v \eta_{y y}, \eta_{y} v_{y}, v v_{y}, \eta \eta_{y}$ and $\eta v$, while the nonlinear terms on the right-hand side of (3.24) are of the form $\eta^{2}, v v_{y y}, v \eta_{y}$ and $\eta v_{y}$; both $N_{1}$ and $N_{2}$ are given explicitly in Appendix A.

From (3.19), (3.20) the boundary conditions $u=v=w=0$ give $v^{\alpha \beta}=v_{v}^{\alpha \beta}=\eta^{\alpha \beta}=0$ on $y= \pm 1$. Thus our system (3.23), (3.24) is written in terms of $v$ and $\eta$ only. Although this system seems very complicated we will find that for most of the analysis we are interested only in the linearized equations, and when we do move on to consider the nonlinear terms all that is needed is to estimate their order of magnitude.

We are mainly interested in the threshold amplitudes for transition for the case of periodic boundary conditions in $x$ and $z$, with period $l_{x}$ and $l_{z}$ respectively, which we take to be of order one as $R \rightarrow \infty$. We will briefly consider how the non-periodic case differs from this in $\S 7.3$.

In the periodic case it is useful to separate the streamwise modes (with zero streamwise wavenumber) from the oblique modes (with non-zero streamwise wavenumber), so that (3.23), (3.24) become

$$
\begin{gathered}
L_{1}^{0 m} v^{0 m}=\sum_{\substack{n_{1}+n_{2}=0 \\
m_{1}+m_{2}=m}} N_{1}^{n_{1} m_{1} n_{2} m_{2}}\left(v^{n_{1} m_{1}}, \eta^{n_{1} m_{1}}, v^{n_{2} m_{2}}, \eta^{n_{2} m_{2}}\right), \\
L_{2}^{0 m}\left(\eta^{0 m}, v^{0 m}\right)=\sum_{\substack{n_{1}+n_{2}=0 \\
m_{1}+m_{2}=m}} N_{2}^{n_{1} m_{1} n_{2} m_{2}}\left(v^{n_{1} m_{1}}, \eta^{n_{1} m_{1}}, v^{n_{2} m_{2}}, \eta^{n_{2} m_{2}}\right), \\
L_{1}^{n m} v^{n m}=\sum_{\substack{n_{1}+n_{2}=n \\
m_{1}+m_{2}=m}} N_{1}^{n_{1} m_{1} n_{2} m_{2}}\left(v^{n_{1} m_{1}}, \eta^{n_{1} m_{1}}, v^{n_{2} m_{2}}, \eta^{n_{2} m_{2}}\right), \\
L_{2}^{n m}\left(\eta^{n m}, v^{n m}\right)=\sum_{\substack{n_{1}+n_{2}=n \\
m_{1}+m_{2}=m}} N_{2}^{n_{1} m_{1} n_{2} m_{2}}\left(v^{n_{1} m_{1}}, \eta^{n_{1} m_{1}}, v^{n_{2} m_{2}}, \eta^{n_{2} m_{2}}\right),
\end{gathered}
$$

where without risk of confusion we have replaced the superscript $\alpha=2 \pi n / l_{x}, \beta=$ $2 \pi \mathrm{m} / l_{z}$ with the superscript $\mathrm{nm}$. Equations (3.25) and (3.26) correspond to equations (2.6) and (2.7) of the toy model in $\S 2$, while equations (3.27) and (3.28) correspond to equations (2.8) and (2.9). The reason for the choice of the nonlinear terms in (2.6)-(2.9) now becomes clear: products of oblique modes appear on the right-hand side of (3.25) and (3.26), while streamwise modes appear on the right-hand side of (3.27) and (3.28) only as a product with an oblique mode.

The natural separation between streamwise and oblique modes occurs because, as we shall see in $\S 4$, the timescale for the evolution of streamwise modes in (3.25) and (3.26) is $R$ (so that $\epsilon$ of $\S 2$ is $R^{-1}$ ), while that for the evolution of the oblique modes in (3.27) and (3.28) is $R^{1 / 3}$ (so that $\delta$ of $\S 2$ is $R^{-1 / 3}$ ).

We need to choose a norm with which to measure the amplitude of a solution. 
We will consider both the $L^{\infty}$ norm, which measures the maximum amplitude of the perturbation

$$
\|\boldsymbol{u}\|_{\infty}=\max _{x, y, z}\{|u|,|v|,|w|\},
$$

and the $L^{2}$ or energy norm, which measures the total energy of the perturbation, and for periodic solutions is

$$
\|\boldsymbol{u}\|_{2}=\left(\frac{1}{2 V} \int_{\text {period in } x, z} \int_{-1}^{1}\left(u^{2}+v^{2}+w^{2}\right) \mathrm{d} y \mathrm{~d} x \mathrm{~d} z\right)^{1 / 2},
$$

where $V$ is the volume of integration. For a single mode, using (3.19), (3.20), these become

and

$$
\|\boldsymbol{u}\|_{\infty}=\max _{x, y, z}\left\{\frac{1}{k^{2}}\left|\alpha \frac{\mathrm{d} v^{\alpha \beta}}{\mathrm{d} y}-\beta \eta^{\alpha \beta}\right|,\left|v^{\alpha \beta}\right|, \frac{1}{k^{2}}\left|\beta \frac{\mathrm{d} v^{\alpha \beta}}{\mathrm{d} y}+\alpha \eta^{\alpha \beta}\right|\right\}
$$

$$
\|\boldsymbol{u}\|_{2}=\left(\frac{1}{8 k^{2}} \int_{-1}^{1}\left(\left(\frac{\mathrm{d} v^{\alpha \beta}}{\mathrm{d} y}\right)^{2}+k^{2}\left(v^{\alpha \beta}\right)^{2}+\left(\eta^{\alpha \beta}\right)^{2}\right) \mathrm{d} y\right)^{1 / 2} .
$$

In fact for the solutions we are interested in we will find that both these norms scale in the same way.

There are four steps that we need to complete in order to calculate the threshold amplitude for transition, namely we need to know

(i) how much transient growth there is in streamwise modes;

(ii) how much transient growth there is in oblique modes;

(iii) how large a streamwise vortex is produced during the growth and decay of an oblique mode;

(iv) how large a streamwise streak has to grow in order to produce a secondary instability.

In the next section we will investigate (i) and (ii). In $\S 5$ we consider (iv), by examining the eigenvalues and eigenfunctions of the linearized equations asymptotically as $R \rightarrow \infty$. Then, in $\S 7$, we consider (iii), and combine the four results to determine the threshold amplitudes for transition.

\section{Transient growth of the linearized equations}

We need to calculate the transient growth of the linearized system

$$
\begin{gathered}
-\left(\frac{\partial^{2}}{\partial y^{2}}-k^{2}\right) \frac{\partial v}{\partial t}=-\left(\frac{\partial^{2}}{\partial y^{2}}-k^{2}\right)^{2} \frac{v}{R}+\mathrm{i} \alpha U\left(\frac{\partial^{2}}{\partial y^{2}}-k^{2}\right) v-\mathrm{i} \alpha \frac{\mathrm{d}^{2} U}{\mathrm{~d} y^{2}} v, \\
\frac{\partial \eta}{\partial t}=-\mathrm{i} \beta \frac{\mathrm{d} U}{\mathrm{~d} y} v-\mathrm{i} \alpha U \eta+\left(\frac{\partial^{2}}{\partial y^{2}}-k^{2}\right) \frac{\eta}{R}
\end{gathered}
$$

with boundary conditions

$$
\begin{gathered}
v( \pm 1, t)=0, \\
\frac{\partial v}{\partial y}( \pm 1, t)=0, \\
\eta( \pm 1, t)=0,
\end{gathered}
$$

where $k^{2}=\alpha^{2}+\beta^{2}$, and we have dropped the superscript $\alpha \beta$ for ease of notation. 
If the period in $x$ is finite as $R \rightarrow \infty$ there are two cases to consider, namely the streamwise mode $\alpha=0$, and the oblique modes $\alpha \neq 0$.

On an infinite domain the relevant cases to consider are $\alpha=O(1 / R)$ and $\alpha \gg 1 / R$; we refer to the former as the streamwise modes (even though they vary slowly with position down the channel) and the latter as the oblique modes.

\subsection{Transient growth of streamwise modes}

We set $\alpha=\bar{\alpha} / R$ with $\bar{\alpha}$ of order one. Then both (4.1) and (4.2) give evolution on a long timescale of order $R$, so we also rescale time by setting $t=R \bar{t}$, giving

$$
\begin{gathered}
-\left(v_{y y}-\beta^{2} v\right)_{\bar{t}}-\mathrm{i} \bar{\alpha} U\left(v_{y y}-\beta^{2} v\right)+\mathrm{i} \bar{\alpha} U^{\prime \prime} v+v_{y y y y}-2 \beta^{2} v_{y y}+\beta^{4} v=0, \\
\eta_{\bar{t}}+\mathrm{i} \bar{\alpha} U \eta+\mathrm{i} R \beta U^{\prime} v-\left(\eta_{y y}-\beta^{2} \eta\right)=0 .
\end{gathered}
$$

Neither equation is now singularly perturbed, so that both $v$ and $\eta$ will vary on an order-one lengthscale. From (4.7) we see that the correct relative scaling of $\eta$ and $v$ is $\eta=O(R v)$. Hence an initial condition of the form $(v, \eta)=\left(v_{0}, 0\right)$ will have transient growth in $\eta$ to order $R v_{0}$ (so that there is growth in the norm of the solution by a factor of order $R$ ) over a timescale of order $R$.

We are now in a position to calculate the threshold amplitude for the transition mechanism (iii) involving only streamwise modes. If we consider the nonlinear terms in (A 1) we see that each is of order $v^{2}, \alpha^{2} \eta^{2}$ or $\alpha v \eta$, while the largest nonlinear term in (A 2) is of order $\alpha \eta^{2}$. Hence in (3.23) the nonlinear terms are of order $v_{0}^{2}$, while the linear part of the equation is of order $v_{0} / R$. Thus the smallest scaling of $v_{0}$ which allows the nonlinear terms to play a role is $v_{0}=O\left(R^{-1}\right)$. In (3.24) the nonlinear terms are of order $R v_{0}^{2}$, but the linear part of the equation is of order $v_{0}$, which again gives a threshold amplitude of order $R^{-1}$. Thus the threshold amplitude for a transition involving only streamwise modes is $O\left(R^{-1}\right)$ in the $L^{\infty}$ norm. Since these modes vary on an order-one lengthscale in $y$, the threshold norm for periodic solutions is also $O\left(R^{-1}\right)$.

This threshold amplitude for a purely streamwise transition is effectively contained in Benney \& Chow (1985), although it is not mentioned explicitly, and they consider the interaction of only two modes in the crosswise direction, $z$.

The reason the threshold is so large even though there is very strong transient growth of the linearized equations is the weak mutual interaction between streamwise modes, because of the factor of $\alpha^{2}$ on the right-hand side of (3.23). Much stronger coupling occurs between streamwise modes and oblique modes. Oblique modes also exhibit linear transient growth, but it is much weaker than for streamwise modes. Let us now examine the transient growth of oblique modes.

\subsection{Transient growth of oblique modes}

\subsubsection{WKB analysis of long-time behaviour}

With $\alpha R \gg 1$ and the natural wKB ansatz $v=\alpha A \exp \left((\alpha R)^{1 / 2} \phi\right), \eta=B \exp \left((\alpha R)^{1 / 2} \phi\right)$, the natural timescale on which to consider long-time behaviour is $t=\alpha^{-1}(\alpha R)^{1 / 2} \bar{t}$, giving

$$
\begin{gathered}
(\alpha R)^{-1 / 2} v_{y y \bar{t}}-k^{2}(\alpha R)^{-1 / 2} v_{t}=(\alpha R)^{-1}\left(v_{y y y y}-2 k^{2} v_{y y}+k^{4} v\right)-\mathrm{i} U\left(v_{y y}-k^{2} v\right)+\mathrm{i} U^{\prime \prime} v, \\
(\alpha R)^{-1 / 2} \eta_{\bar{t}}=-\mathrm{i} \beta U^{\prime} v-\mathrm{i} U \eta+(\alpha R)^{-1}\left(\eta_{y y}-k^{2} \eta\right),
\end{gathered}
$$

so that at leading order

$$
\left(\phi_{y}\right)^{2} \phi_{\bar{t}}=\left(\phi_{y}\right)^{4}-\mathrm{i} U\left(\phi_{y}\right)^{2},
$$




$$
B \phi_{\bar{t}}=-\mathrm{i} \beta A-\mathrm{i} U B+B\left(\phi_{y}\right)^{2} .
$$

However, we see immediately that (4.10), (4.11) imply $A=0$, which implies that our scaling for $v$ is incorrect. We must set instead $v=\alpha(\alpha R)^{-1 / 2} A \exp \left((\alpha R)^{1 / 2} \phi\right)$, so that $A$ will appear at the next order in the expansion of the equation for $\eta$. This happens because with the WKB ansatz $\eta$ satisfies the same equation as $v_{y y}$ to leading order, which is another manifestation of non-normality.

Writing $p=\phi_{y}, q=\phi_{t}$, the eikonal equation (4.10) is

$$
q-p^{2}+\mathrm{i} U=0,
$$

for which Charpit's equations are

$$
\dot{y}=-2 p, \quad \dot{\bar{t}}=1, \quad \dot{p}=-\mathrm{i} U^{\prime}, \quad \dot{q}=0, \quad \dot{\phi}=-2 p^{2}+q,
$$

with initial data

$$
y_{0}=s, \quad t_{0}=0, \quad p_{0}=\phi_{0}^{\prime}, \quad q_{0}=\left(\phi_{0}^{\prime}\right)^{2}-\mathrm{i} U(s)
$$

and solution

$$
\begin{aligned}
q & =\left(\phi_{0}^{\prime}\right)^{2}-\mathrm{i} U(s), \\
\bar{t} & =\tau, \\
p & =\phi_{0}^{\prime}-\mathrm{i} U^{\prime}(s) \tau, \\
y & =-2 \phi_{0}^{\prime} \tau+\mathrm{i} U^{\prime}(s) \tau^{2}+s, \\
\phi & =\phi_{0}-\mathrm{i} U(s) \tau-\left(\phi_{0}^{\prime}\right)^{2} \tau+2 \mathrm{i} U^{\prime}(s) \phi_{0}^{\prime} \tau^{2}+\frac{2 U^{\prime}(s)^{2} \tau^{3}}{3} .
\end{aligned}
$$

Since we are considering subcritical transition, we are interested in parameter values for which all the eigenvalues of (4.1) have negative imaginary part, so that for large times the solution must decay, i.e. $\operatorname{Re}(\phi) \rightarrow-\infty$ as $\bar{t} \rightarrow \infty$. The question is to determine the maximum extent of any transient growth. If $\operatorname{Re}(\phi)>\operatorname{Re}\left(\phi_{0}\right)$ for small times then there is exponentially large transient growth. Expanding the WKB solution for small times we find

$$
\begin{aligned}
& \phi \sim \phi_{0}(y)+\bar{t}\left(\left(\phi_{0}^{\prime}\right)^{2}-\mathrm{i} U\right)+\bar{t}^{2} \phi_{0}^{\prime}\left(2 \phi_{0}^{\prime} \phi_{0}^{\prime \prime}-\mathrm{i} U^{\prime}\right) \\
& \quad+\frac{1}{3} \bar{t}^{3}\left(-\left(U^{\prime}\right)^{2}-6 \mathrm{i} U^{\prime} \phi_{0}^{\prime} \phi_{0}^{\prime \prime}+12\left(\phi_{0}^{\prime}\right)^{2}\left(\phi_{0}^{\prime \prime}\right)^{2}+4\left(\phi_{0}^{\prime}\right)^{3} \phi_{0}^{\prime \prime \prime}\right)+\cdots .
\end{aligned}
$$

Now the norm of the solution is determined by the maximum value of $\operatorname{Re}\left(\phi_{0}\right)$. Supposing that this is at $y=y_{0}$, there are two cases to consider.

Suppose first that there is an internal maximum in the solution, so that there is a saddle point of $\phi_{0}+\bar{\phi}_{0}$ at $y_{0}$, where $\bar{\phi}_{0}$ is the complex conjugate of $\phi_{0}$. Then

$$
\phi_{0}^{\prime}\left(y_{0}\right)+\bar{\phi}_{0}^{\prime}\left(y_{0}\right)=0 \text {, }
$$

so that $\phi_{0}^{\prime}\left(y_{0}\right)$ is pure imaginary. Hence the real part of the coefficient of $\bar{t}$ in (4.18) is negative, giving exponential decay on an $O\left(\alpha^{-1}\right)$ timescale (since $\bar{t}(\alpha R)^{1 / 2}=O(1) \Rightarrow$ $t=O\left(\alpha^{-1}\right)$ ), unless $\phi_{0}^{\prime}\left(y_{0}\right)=0$. In this case the coefficient of $\bar{t}^{2}$ is also zero, so that the norm is determined by the $\bar{t}^{3}$ term, which is $-\left(U^{\prime}\right)^{2} / 3$. The solution still ultimately decays, but now over a longer timescale $\alpha^{-1}(\alpha R)^{1 / 3}\left(\right.$ since $\bar{t}^{3}(\alpha R)^{1 / 2}=O(1) \Rightarrow t=$ $\left.O\left(\alpha^{-1}(\alpha R)^{1 / 3}\right)\right)$.

Now suppose that the maximum occurs at an endpoint. In this case, in the ray solution (4.13)-(4.17) we have to be careful that we do not include irrelevant rays originating from the analytic continuation of the solution outside the domain, which 
may lead to spurious exponential growth $\uparrow$. However, when the solution is dominated by the endpoint we may do a local analysis in its vicinity, and the solution will be a collection of wall modes (which we will discuss in $\S 5$ ). These have eigenvalues with imaginary part $O\left(\alpha(\alpha R)^{-1 / 3}\right)$, and have transient growth on this timescale, but only exponential decay on the timescale of $\alpha^{-1}(\alpha R)^{1 / 2}$.

In either case we see that on the timescale of $\alpha^{-1}(\alpha R)^{1 / 2}$ there is no transient growth, only exponential decay. The best we can hope for is to make this decay as slow as possible, giving a chance for some algebraic transient growth. Suppose therefore, from the discussion earlier, that $\phi_{0}^{\prime}\left(y_{0}\right)=0$, so that for small times

$$
v \sim A \exp \left(-(\alpha R)^{1 / 2} \bar{t}^{3}\left(U^{\prime}\right)^{2} / 3\right) .
$$

To observe the algebraic growth we should rescale $\bar{t}$ with $(\alpha R)^{-1 / 6}$ so that the new timescale is given by $t=\alpha^{-1}(\alpha R)^{1 / 3} \tilde{t}$. Thus we are led to the following key parameter regime:

4.2.2. Transient growth on the timescale of $\alpha^{-1}(\alpha R)^{1 / 3}$

With $t=\alpha^{-1}(\alpha R)^{1 / 3} \tilde{t}$ we have

$$
\begin{gathered}
(\alpha R)^{-1 / 3} v_{y y \tilde{t}}-k^{2}(\alpha R)^{-1 / 3} v_{\tilde{t}}=(\alpha R)^{-1}\left(v_{y y y y}-2 k^{2} v_{y y}+k^{4} v\right)-\mathrm{i} U\left(v_{y y}-k^{2} v\right)+\mathrm{i} U^{\prime \prime} v, \\
(\alpha R)^{-1 / 3} \eta_{\tilde{t}}=-\mathrm{i} \beta \alpha^{-1} U^{\prime} v-\mathrm{i} U \eta+(\alpha R)^{-1}\left(\eta_{y y}-k^{2} \eta\right) .
\end{gathered}
$$

As before, $v$ is an order of magnitude smaller than $\eta$, so we set $v=\alpha(\alpha R)^{-1 / 3}$ $A \exp \left((\alpha R)^{1 / 3} \phi\right), \eta=B \exp \left((\alpha R)^{1 / 3} \phi\right)$. Then at leading order the eikonal equation is $\phi_{\tilde{t}}=-\mathrm{i} U$. Hence

$$
\phi=\phi_{0}(y)-\mathrm{i} U \tilde{t} .
$$

The amplitude equations for $A$ and $B$ are

$$
\begin{aligned}
\left(\phi_{y}\right)^{2} A_{\tilde{t}}-2 \phi_{y} \mathrm{i} U^{\prime} A- & \left(\phi_{y}\right)^{4} A=(\alpha R)^{-1 / 3}\left(-2 \mathrm{i} U^{\prime} A_{y}-4\left(\phi_{y}\right)^{3} A_{y}\right. \\
& \left.+2 \phi_{y} A_{y \tilde{t}}+\phi_{y y} A_{\tilde{t}}-6\left(\phi_{y}\right)^{2} \phi_{y y} A\right)+O\left((\alpha R)^{-2 / 3}\right), \\
B_{\tilde{t}}-\left(\phi_{y}\right)^{2} B+\mathrm{i} \beta U^{\prime} A= & (\alpha R)^{-1 / 3}\left(-2 \phi_{y} B_{y}-\phi_{y y} B\right)+O\left((\alpha R)^{-2 / 3}\right) .
\end{aligned}
$$

At leading order we have

$$
\begin{gathered}
\phi_{y} A_{\tilde{t}}=2 \mathrm{i} U^{\prime} A+\left(\phi_{y}\right)^{3} A, \\
B_{\tilde{t}}=\left(\phi_{y}\right)^{2} B-\mathrm{i} \beta U^{\prime} A .
\end{gathered}
$$

Let $\tau=U^{\prime} \tilde{t}+\mathrm{i} \phi_{0}^{\prime}(y)$, so that $\phi_{y}=-\mathrm{i} \tau$. Then

$$
\begin{gathered}
U^{\prime}\left(A \tau^{2}\right)_{\tau}=-\tau^{4} A, \\
U^{\prime} B_{\tau}=-\tau^{2} B-\mathrm{i} U^{\prime} A .
\end{gathered}
$$

Hence

$$
\begin{gathered}
A=\frac{a_{0}(y) \exp \left(-\tau^{3} /\left(3 U^{\prime}\right)\right)}{\tau^{2}}, \\
B=\left(\frac{\mathrm{i} \beta a_{0}(y)}{\tau}+b_{0}(y)\right) \exp \left(-\tau^{3} /\left(3 U^{\prime}\right)\right) .
\end{gathered}
$$

$\dagger$ In fact there will be 'diffracted' rays fanning out from each endpoint, and these will determine which rays from the analytically continued boundary should be included via Stokes' phenomenon, as described in Chapman et al. (1999). This is also true away from the endpoints when we consider order-one $\bar{t}$ rather than small $\bar{t}$ as above. 
Now, $A$ and $B$ blow up as $\tau \rightarrow 0$. The growth is capped when the lower-order terms on the right-hand side of (4.22) become as large as the leading-order terms which were retained in (4.24), and therefore cease to be negligible. Let us calculate asymptotically how close $\tau$ needs to be to zero for this to happen.

As $\tau \rightarrow 0$, the dominant terms on the left-hand side of (4.22) are of order $1 / \tau$. The point at which the blow-up occurs is the value of $y_{0} \in[-1,1]$ which leads to the smallest real value of $-\mathrm{i} \phi_{0}^{\prime}\left(y_{0}\right) /\left(\alpha U^{\prime}\left(y_{0}\right)\right)$, which is the blow-up time $\tilde{t}_{0}$. Now, if $\phi_{0}^{\prime \prime}\left(y_{0}\right) U^{\prime}\left(y_{0}\right) \neq \phi_{0}^{\prime}\left(y_{0}\right) U^{\prime \prime}\left(y_{0}\right)$ then $\phi_{y y}\left(y_{0}\right)$ does not tend to zero as $\tilde{t} \rightarrow \tilde{t}_{0}$. The right-hand side of (4.22) is then of order $(\alpha R)^{-1 / 3} / \tau^{3}$ as $\tau \rightarrow 0$. Hence the neglected terms become important when $\tau=(\alpha R)^{-1 / 6}$, so that the growth in $A$ is capped at $(\alpha R)^{1 / 3}$ and in $B$ at $(\alpha R)^{1 / 6}$. However, if $\phi_{0}^{\prime \prime}\left(y_{0}\right) U^{\prime}\left(y_{0}\right)=\phi_{0}^{\prime}\left(y_{0}\right) U^{\prime \prime}\left(y_{0}\right)$ then $\phi_{y y}$ is order $\tau$ as $\tau \rightarrow 0$, and the right-hand side of (4.22) is of order $(\alpha R)^{-1 / 3} / \tau^{2}$. In this case the neglected terms become important when $\tau=(\alpha R)^{-1 / 3}$, so that the growth in $A$ is capped at $(\alpha R)^{2 / 3}$ and in $B$ at $(\alpha R)^{1 / 3}$.

Thus there is algebraic transient growth on a timescale of order $\alpha^{-1}(\alpha R)^{1 / 3}$. Note, though, that this transient growth is not of the form $t^{m} \mathrm{e}^{-t}$, as would be expected from a degenerate eigenvalue (that is, a repeated eigenvalue with multiplicity higher than the dimension of its eigenspace), nor of the form $\mathrm{e}^{-t}-\mathrm{e}^{-2 t}$, which would be expected from a pair of almost parallel eigenvectors, as in the toy model of $\S 2$. The functions $v$ and $\eta$ stay of order $\alpha(\alpha R)^{-1 / 3}$ and order one respectively for a time of order $\alpha^{-1}(\alpha R)^{1 / 3}$, then suddenly, over a short (i.e. $\left.O\left(\alpha^{-1}\right)\right)$ time, they grow to be orders $\alpha(\alpha R)^{1 / 3}$ and $(\alpha R)^{1 / 3}$ respectively.

Interpreting the transient growth in terms of eigenfunctions requires us to consider not one or two but all the eigenfunctions. The transient growth occurs because the terms in the eigenfunction expansion suddenly all come into phase. The effect may be illustrated by considering the sum

$$
\sum_{n} a_{n} \mathrm{e}^{\mathrm{i} \lambda_{n} t}
$$

for a set of 'eigenvalues' $\lambda_{n}$ which become dense on a line, so that in the limit we have the approximation

$$
\int a(\lambda) \mathrm{e}^{\mathrm{i} \lambda t} \mathrm{~d} \lambda
$$

Now if we choose $a(\lambda)=\mathrm{e}^{-\mathrm{i} \lambda t_{0}}$ then

$$
\int a(\lambda) \mathrm{e}^{\mathrm{i} \lambda t} \mathrm{~d} \lambda \sim \frac{1}{\mathrm{i}\left(t-t_{0}\right)},
$$

and there is a blow-up as all the 'eigenfunctions' come into phase at $t=t_{0}$.

We have seen that the maximum growth in $A$ and $B$ occurs when $\phi_{0}^{\prime \prime}\left(y_{0}\right) U^{\prime}\left(y_{0}\right)=$ $\phi_{0}^{\prime}\left(y_{0}\right) U^{\prime \prime}\left(y_{0}\right)$. However, to compare with numerical results it is interesting to calculate the energy norm of the solution. Before the blow-up time we have

$$
v \sim \alpha(\alpha R)^{-1 / 3} \frac{a_{0}(y) \exp \left(-\left(\mathrm{i} \phi_{0}+U^{\prime} \hat{t}\right)^{3} /\left(3 U^{\prime}\right)\right)}{\left(\mathrm{i} \phi_{0}^{\prime}+U^{\prime} \hat{t}\right)^{2}} \exp \left((\alpha R)^{1 / 3}\left(\phi_{0}-\mathrm{i} U \hat{t}\right)\right),
$$

with

$$
v \sim-\alpha(\alpha R)^{-1 / 3} \frac{a_{0}(y) \exp \left(\mathrm{i} \phi_{0}^{3} /\left(3 U^{\prime}\right)\right)}{\left(\phi_{0}^{\prime}\right)^{2}} \exp \left((\alpha R)^{1 / 3} \phi_{0}\right)
$$

initially. First, suppose the initial phase $\phi_{0}$ is such that $\phi_{0}+\bar{\phi}_{0}$ has a saddle point at 

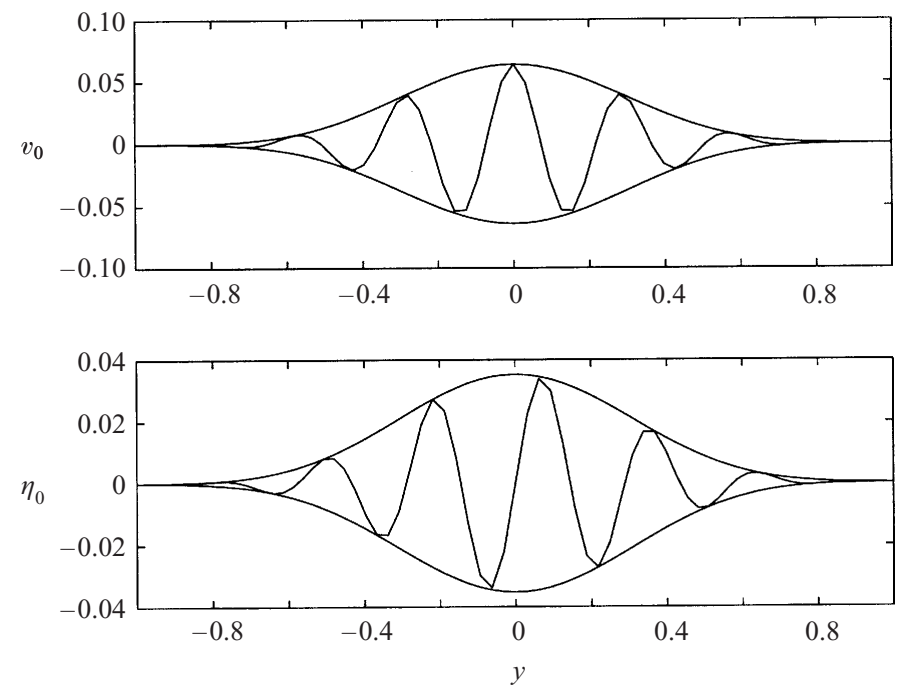

FIGURE 4. Numerically determined initial condition leading to maximal growth for plane Couette flow with $\alpha=\beta=1, R=10000$. The inner lines show the real part of the function, while the outer lines show its absolute value. The linearized equations were solved in MATLAB using codes written by Satish Reddy based a Chebyshev hybrid spectral discretization, as described in Reddy \& Henningson (1993) and Reddy et al. (1993). The initial condition leading to the maximum transient growth was determined through a singular value decomposition of $\exp \left(A t^{*}\right)$, where the linearized system is $\boldsymbol{y}_{t}=A \boldsymbol{y}$, and $t^{*}$ is the time at which the norm of $\exp (A t)$ is maximum.

$y_{0}$ which dominates the $L^{2}$ norm of $v$, and that $\phi_{0}(y)-\phi_{0}\left(y_{0}\right) \sim \mathrm{i} a\left(y-y_{0}\right)+\mathrm{i} b\left(y-y_{0}\right)^{2}$ $-c\left(y-y_{0}\right)^{2 n}$ as $y \rightarrow y_{0}$, with $a$ and $b$ real and $c$ not pure imaginary. When performing the method of steepest descents on the $L^{2}$ norm of the initial condition the dominant contribution comes from a region $y-y_{0}=O\left((\alpha R)^{-1 /(6 n)}\right)$, giving an $L^{2}$ norm of $v$ of order $(\alpha R)^{-1 /(6 n)}$ times the $L^{\infty}$ norm. Now the denominator of $v$ at the blow-up time looks like $\left(\left(U^{\prime \prime}\left(y_{0}\right) \tilde{t}_{0}-2 b\right)\left(y-y_{0}\right)-2 n c i\left(y-y_{0}\right)^{2 n-1}\right)^{2}$ as $y \rightarrow y_{0}$. To maximize the $L^{2}$ norm we need to maximize the region over which the denominator is of the order of $(\alpha R)^{-2 / 3}$ (which is the cap as we have seen). Hence we should choose $b=U^{\prime \prime}\left(y_{0}\right) \tilde{t}_{0} / 2$, giving $y-y_{0}=O\left((\alpha R)^{-1 /(6 n-3)}\right)$. Hence the maximum $L^{2}$ norm of $v$ is of the order of $(\alpha R)^{-1 /(6 n-3)}$ times the maximum $L^{\infty}$ norm. Thus we conclude that the growth in the $L^{2}$ norm is less than that in the $L^{\infty}$ norm if the $L^{2}$ norm of $v$ is dominated by a saddle point of $\phi_{0}+\bar{\phi}_{0}$.

However, we can achieve the same growth in the $L^{2}$ norm as in the $L^{\infty}$ norm if we take $\phi_{0}$ to be pure imaginary so that the norm of $v$ is not dominated by a particular $y_{0}$, but each $y$ contributes equally. Then, clearly, to maximize the growth we need the blow-up times of all points $y$ to be the same, so that $A \rightarrow \infty$ for all $y$ as $\tilde{t} \rightarrow \tilde{t}_{0}$. Hence, for maximal growth, we should choose $\phi_{0}=i \tilde{t}_{0} U$, where $\tilde{t}_{0}$ is real. Even more importantly, we will see later that choosing $\phi_{0}$ to be pure imaginary also gives the maximum nonlinear coupling between oblique modes and streamwise modes.

In figure 4 we show the numerically determined initial condition leading to the maximum transient growth in plane Couette flow for the case $\alpha=\beta=1, R=10000$. The outer lines show the absolute value of the function, while the inner line shows its real part. In figure 5 we show the phase of the solution by plotting $(\alpha R)^{-1 / 3} \operatorname{Im}(\log (v))$ and $(\alpha R)^{-1 / 3} \operatorname{Im}(\log (\eta))$. We also plot $U=y$ for reference; we see that the numerically determined maximal initial condition is close to the asymptotic one. In figure 6 we 


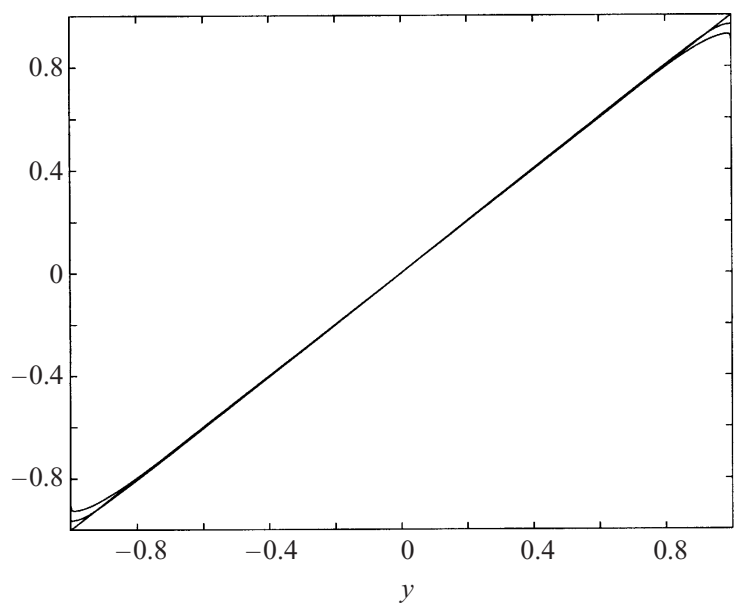

FiguRE 5. Numerically determined phase of the initial condition leading to maximal growth for plane Couette flow with $\alpha=\beta=1, R=10000$.

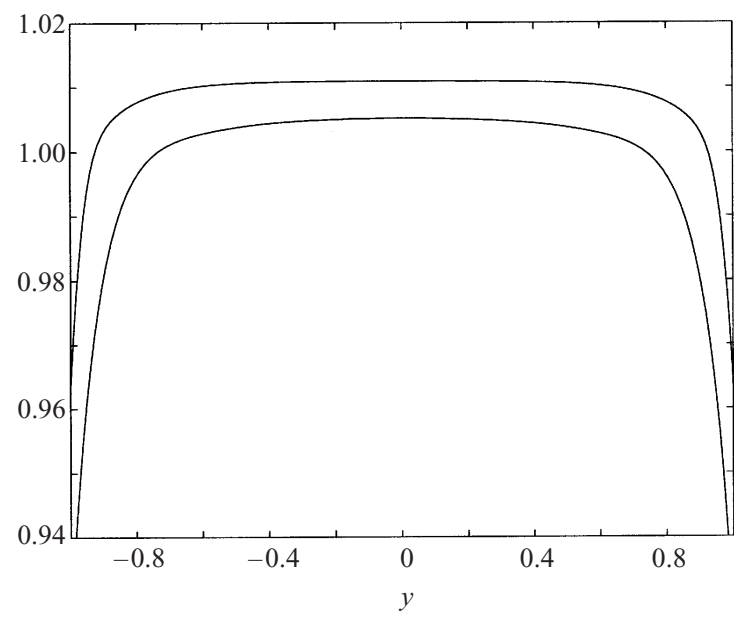

FIGURE 6. Numerically determined phase of the initial condition leading to maximal growth for plane Couette flow with $\alpha=\beta=1, R=10000$, divided by the asymptotically determined value $U$. The ratio should be the constant $\tilde{t}_{0}$.

show $(\alpha R)^{-1 / 3} \operatorname{Im}(\log (v)) / U$ and $(\alpha R)^{-1 / 3} \operatorname{Im}(\log (\eta)) / U$; we see that in this case $\tilde{t}_{0}$ is very close to unity. However, its precise value is not important for determining the scaling of the threshold amplitude.

\subsubsection{Evolution through the blow-up time}

Let us now consider the complete evolution of the solution with an initial condition of the form $\phi_{0}=i \tilde{t}_{0} U$, including the transition through the blow-up region. For times $0 \leqslant \tilde{t}<\tilde{t}_{0}$ we have

$$
\begin{gathered}
v \sim \alpha(\alpha R)^{-1 / 3} \frac{a_{0}(y) \exp \left(-\left(U^{\prime}\right)^{2}\left(\tilde{t}-\tilde{t}_{0}\right)^{3} / 3\right)}{\left(U^{\prime}\right)^{2}\left(\tilde{t}-\tilde{t}_{0}\right)^{2}} \exp \left(\mathrm{i} U(\alpha R)^{1 / 3}\left(\tilde{t}_{0}-\tilde{t}\right)\right), \\
\eta \sim\left(\frac{\mathrm{i} \beta a_{0}(y)}{U^{\prime}\left(\tilde{t}-\tilde{t}_{0}\right)}+b_{0}(y)\right) \exp \left(-\left(U^{\prime}\right)^{2}\left(\tilde{t}-\tilde{t}_{0}\right)^{3} / 3\right) \exp \left(\mathrm{i} U(\alpha R)^{1 / 3}\left(\tilde{t}_{0}-\tilde{t}\right)\right) .
\end{gathered}
$$


We have seen that the growth should be capped when $\tilde{t}-\tilde{t}_{0}=O\left((\alpha R)^{-1 / 3}\right)$. We use the method of matched asymptotic expansions to examine the evolution for $\tilde{t}$ close to $\tilde{t}_{0}$. We set $\tilde{t}=\tilde{t}_{0}+\hat{t}(\alpha R)^{-1 / 3}, v=\alpha(\alpha R)^{1 / 3} \hat{v}$, and $\eta=(\alpha R)^{1 / 3} \hat{\eta}$. In (4.19), (4.20) this gives

$$
\begin{gathered}
\hat{v}_{y y \hat{t}}-k^{2} \hat{v}_{\hat{t}}=(\alpha R)^{-1}\left(\hat{v}_{y y y y}-2 k^{2} \hat{v}_{y y}+k^{4} \hat{v}\right)-\mathrm{i} U\left(\hat{v}_{y y}-k^{2} \hat{v}\right)+\mathrm{i} U^{\prime \prime} \hat{v}, \\
\hat{\eta}_{\hat{t}}=-\mathrm{i} \beta U^{\prime} \hat{v}-\mathrm{i} U \hat{\eta}+(\alpha R)^{-1}\left(\hat{\eta}_{y y}-k^{2} \hat{\eta}\right) .
\end{gathered}
$$

Matching with the 'outer' solution (4.32)-(4.33) gives the conditions

$$
\begin{aligned}
& \hat{v} \sim \frac{a_{0}(y)}{\left(U^{\prime}\right)^{2} \hat{t}^{2}} \mathrm{e}^{-\mathrm{i} U \hat{t}}, \\
& \hat{\eta} \sim \frac{\mathrm{i} \beta a_{0}(y)}{U^{\prime} \hat{t}} \mathrm{e}^{-\mathrm{i} U \hat{t}}
\end{aligned}
$$

as $\hat{t} \rightarrow-\infty$. Note that $v$ and $\eta$ cease to oscillate rapidly in $y$ as they reach their maximum.

Now, to leading order in (4.34)-(4.35)

$$
\begin{aligned}
\hat{v}_{y y \hat{t}}-k^{2} \hat{v}_{\hat{t}} & =-\mathrm{i} U\left(\hat{v}_{y y}-k^{2} \hat{v}\right)+\mathrm{i} U^{\prime \prime} \hat{v}, \\
\hat{\eta}_{\hat{t}} & =-\mathrm{i} \beta U^{\prime} \hat{v}-\mathrm{i} U \hat{\eta} .
\end{aligned}
$$

If $\hat{v}$ was known, we could solve (4.39) for $\hat{\eta}$ to give

$$
\hat{\eta}=-\mathrm{i} \beta U^{\prime} \mathrm{e}^{-\mathrm{i} U \hat{t}} \int_{-\infty}^{\hat{t}} \hat{v} \mathrm{e}^{\mathrm{i} U \hat{t}} \mathrm{~d} \hat{t} .
$$

Note that using (4.36) we have

$$
\hat{\eta} \sim \mathrm{i} \beta U^{\prime} \mathrm{e}^{-\mathrm{i} U \hat{t}} \frac{a_{0}(y)}{\left(U^{\prime}\right)^{2} \hat{t}},
$$

as $\hat{t} \rightarrow-\infty$, in agreement with (4.37). As $\hat{t} \rightarrow \infty$ we have

$$
\hat{\eta} \sim-\mathrm{i} \beta U^{\prime} \mathrm{e}^{-\mathrm{i} U \hat{t}} \int_{-\infty}^{\infty} \hat{v} \mathrm{e}^{\mathrm{i} U \hat{t}} \mathrm{~d} \hat{t}
$$

Unfortunately it is difficult to solve (4.38) for the case of plane Poiseuille flow. However, for plane Couette flow we can solve (4.38) analytically.

\subsubsection{Inner solution for plane Couette flow}

With $U=y$ we may write (4.38) as

$$
\xi_{\hat{t}}=-\mathrm{i} y \xi
$$

where $\xi=\hat{v}_{y y}-k^{2} \hat{v}$. Hence

giving

$$
\xi=\xi_{0}(y) \mathrm{e}^{-\mathrm{i} y \hat{t}},
$$

$$
\hat{v}_{y y}-k^{2} \hat{v}=\xi_{0}(y) \mathrm{e}^{-\mathrm{i} y \hat{t}},
$$

whence

$$
\begin{aligned}
\hat{v}= & \int_{-1}^{y} \frac{\sinh k(x+1) \sinh k(y-1)}{k \sinh 2 k} \xi_{0}(x) \mathrm{e}^{-\mathrm{i} x \hat{t}} \mathrm{~d} x \\
& +\int_{y}^{1} \frac{\sinh k(x-1) \sinh k(y+1)}{k \sinh 2 k} \xi_{0}(x) \mathrm{e}^{-\mathrm{i} x \hat{t}} \mathrm{~d} x .
\end{aligned}
$$


Integrating by parts as $\hat{t} \rightarrow-\infty$ gives

$$
\hat{v} \sim-\frac{\xi_{0}(y) \mathrm{e}^{-\mathrm{i} y \hat{t}}}{\hat{t}^{2}} .
$$

Hence

$$
\xi_{0}(y)=-a_{0}(y) .
$$

The same argument works as $\hat{t} \rightarrow \infty$ to give

$$
\hat{v} \sim \frac{a_{0}(y)}{\hat{t}^{2}} \mathrm{e}^{-\mathrm{i} y \hat{t}} .
$$

Hence, although $v$ reaches a maximum of order $\alpha(\alpha R)^{1 / 3}$, it holds this order only for a short (order-one) time. Once we are back into the outer region $\hat{t}=O\left((\alpha R)^{1 / 3}\right)$, $\tilde{t}=O(1), v$ is again of order $\alpha(\alpha R)^{-1 / 3}$. However, from (4.41) we have

$$
\begin{gathered}
\hat{\eta} \sim-\mathrm{i} \beta \mathrm{e}^{-\mathrm{i} y \hat{t}} \int_{-\infty}^{\infty} \hat{v} \mathrm{e}^{\mathrm{i} y \hat{t}} \mathrm{~d} \hat{t} \\
\sim \eta_{0}(y) \mathrm{e}^{-\mathrm{i} y \hat{t}}
\end{gathered}
$$

as $\hat{t} \rightarrow+\infty$. Since $\eta_{0}$ is not identically zero, $\eta$ remains order $(\alpha R)^{1 / 3}$ into the outer region $\hat{t}=O\left((\alpha R)^{1 / 3}\right)$ and decays slowly over this timescale.

\subsubsection{Inner solution for plane Poiseuille flow}

For plane Poiseuille flow we cannot solve (4.38) analytically. However, the only possible behaviour as $\hat{t} \rightarrow \infty$ is

$$
\hat{v} \sim \frac{\hat{v}_{0}(y)}{\left(U^{\prime}\right)^{2} \hat{t}^{2}} \mathrm{e}^{-\mathrm{i} U \hat{t}},
$$

for some $\hat{v}_{0}(y)$, so that $\hat{v}$ decays at infinity exactly as in plane Couette flow. By writing $\hat{v}$ as $f \mathrm{e}^{-\mathrm{i} U \hat{t}}$ in (4.38) and integrating over time we find that

$$
\hat{\eta}_{0}=\frac{\beta U^{\prime}}{U^{\prime \prime}}\left(\hat{v}_{0}-a_{0}\right) .
$$

Now, for plane Couette flow $\hat{v}_{0}=a_{0}$, but also $U^{\prime \prime}=0$, so that $\hat{\eta}_{0}$ is non-zero. For plane Poiseuille flow we have no reason to suppose that $\hat{v}_{0}=a_{0}$ (although we have been unable to prove $\hat{v}_{0} \neq a_{0}$ ), and therefore we conjecture that in this case also $\hat{\eta}_{0} \neq 0$.

\subsubsection{Times of order $\alpha^{-1}(\alpha R)^{1 / 3}$ after the maximum}

Let us now consider the 'outer' region again for times beyond the blow-up point. We rescale time again with $(\alpha R)^{1 / 3}$.

For plane Couette flow $v$ is now order $\alpha(\alpha R)^{-1 / 3}$, while $\eta$ is still order $(\alpha R)^{1 / 3}$, and we conjecture that this is the case for plane Poiseuille flow also. Then $v$ is too small to appear in the $\eta$-equation at leading order, so that the two equations decouple. Equation (4.20) becomes

$$
(\alpha R)^{-1 / 3} \hat{\eta}_{\tilde{t}}=-\mathrm{i} U \hat{\eta}+(\alpha R)^{-1}\left(\hat{\eta}_{y y}-k^{2} \hat{\eta}\right) .
$$

We use the WKB ansatz $\hat{\eta}=B \exp \left((\alpha R)^{1 / 3} \phi\right)$ as before. Matching from the 'inner' blow-up region gives the initial condition at $\tilde{t}=\tilde{t}_{0}$ as $\phi=0, B=\eta_{0}$. As before, at leading order $\phi_{\tilde{t}}=-\mathrm{i} U$ giving

$$
\phi=-\mathrm{i} U\left(\tilde{t}-\tilde{t}_{0}\right) .
$$



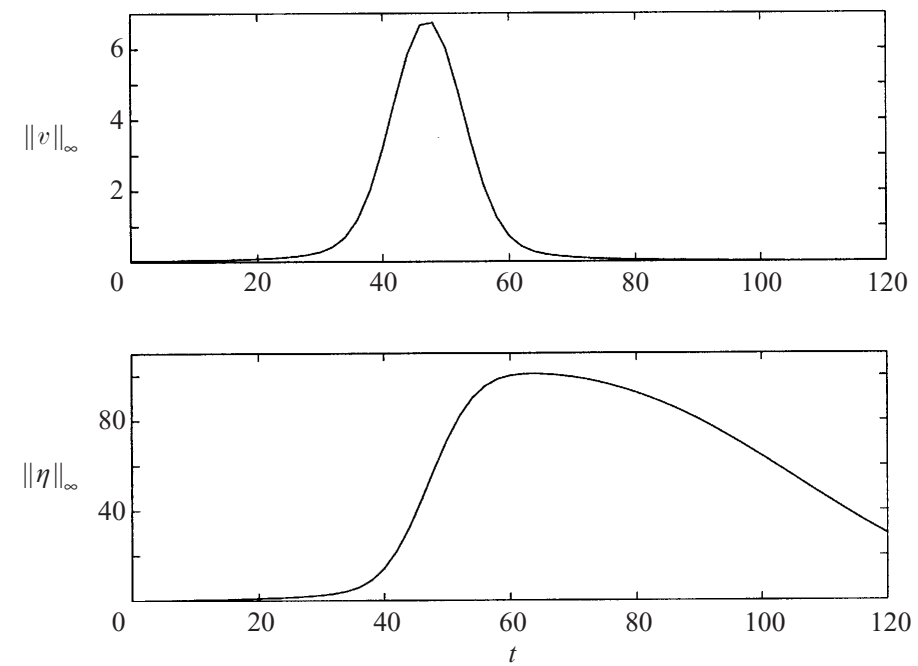

FiguRE 7. Numerical evolution of the maximum value of the numerically determined initial condition leading to maximal growth for plane Couette flow with $\alpha=\beta=1, R=100000$.

At next order

$$
B_{\tilde{t}}=\left(\phi_{y}\right)^{2} B
$$

giving

$$
B=\eta_{0} \exp \left(-\left(U^{\prime}\right)^{2}\left(\tilde{t}-\tilde{t}_{0}\right)^{3} / 3\right)
$$

Hence

$$
\eta \sim \eta_{0}(\alpha R)^{1 / 3} \exp \left(-\alpha^{2}\left(U^{\prime}\right)^{2}\left(\tilde{t}-\tilde{t}_{0}\right)^{3} / 3\right) \exp \left(-\mathrm{i} \alpha U(\alpha R)^{1 / 3}\left(\tilde{t}-\tilde{t}_{0}\right)\right)
$$

This describes the slow decay of $\eta$ over this timescale of order $(\alpha R)^{1 / 3}$. Note that during the decay $\eta$ begins to oscillate rapidly again, so that its derivative is $O\left((\alpha R)^{2 / 3}\right)$.

In figure 7 we show a numerical simulation of the evolution of the maximum of the velocity and vorticity for the numerically determined initial condition leading to maximum transient growth in plane Couette flow with $\alpha=\beta=1$ and $R=100000$. We see that this reproduces the qualitative features of the asymptotic solution, namely that $v$ stays small for a long time, suddenly becomes large, and then just as suddenly returns to be small again, whereas $\eta$ stays small for a long time, suddenly becomes large, and then slowly decays back to zero. Notice that the time that $\eta$ reaches its maximum is delayed slightly behind the time that $v$ reaches its maximum. This is because $\eta$ is effectively the time integral of $v$ (see (4.40)). This delay is $O\left(\alpha^{-1}\right)$ as $\alpha R \rightarrow \infty$.

In figure 8 we show $v$ at the time it has reached its maximum value. The real part of $v$ is shown; the imaginary part is zero. Notice that $v$ is not oscillatory, in agreement with the asymptotics.

\subsection{Asymptotically large $\beta$}

For completeness we should determine the extent of the transient growth in the case when $\beta$ is asymptotically large in $R$. Since this case turns out to be not relevant for the optimal routes to transition we omit the details and simply give an indication of the results.

The case of large $\beta$ seems promising at first, since (4.29) indicates that as $\beta$ increases the scaling $\eta$ increases relative to $v$, which is one cause of the transient growth. The 


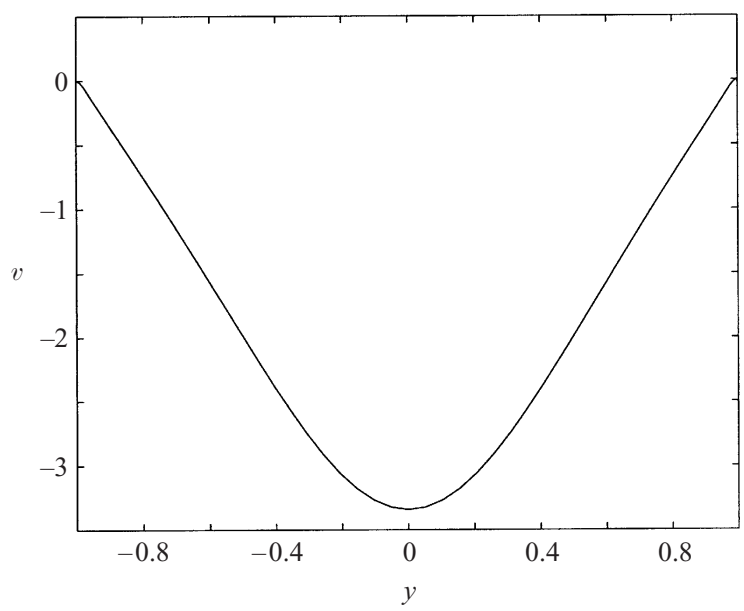

FIGURE 8 . The solution $v$ at its maximum value using the numerically determined initial condition leading to maximal growth for plane Couette flow with $\alpha=\beta=1, R=10000$. The real part of $v$ is shown; the imaginary part is zero.

canonical case to consider is $\beta=R^{1 / 3} \bar{\beta}$, with $\bar{\beta}$ of order one. Besides changing the relative scalings of $v$ and $\eta$ this has the effect of changing the denominator in (4.28) from $\tau^{2}$ to $\tau^{2}+\bar{\beta}^{2}$ (as well as modifying the exponential decay). However, this means that the growth in $v$ is smaller, since the double pole has split into two simple poles. The result is a transient growth of order $R^{1 / 3}$ in $\eta$, which is comparable to that when $\beta$ is of order one. However, to achieve this growth requires $\phi_{0}^{\prime}(y)$ to have real part equal to $\pm \bar{\beta}$ (so that $\tau^{2}+\bar{\beta}^{2}$ reaches zero for real $\tilde{t}$ ). Hence the optimally growing initial condition is localized near the wall. We will see that this localization leads to a smaller coupling with the streamwise modes, so we do not need to consider the case of large $\beta$ further.

\subsection{Summary}

We have now determined the answers to questions (i) and (ii) of $\S 3$, namely we have determined the extent of the transient growth in both streamwise and oblique modes. In the next section we will examine the eigenvalues of the linearized equations, but we first summarize our results so far.

\section{Streamwise modes: $\alpha$ of order $1 / R$}

The streamwise modes exhibit a maximum transient growth of order $R$ over a timescale of order $R$. They vary on an order-one lengthscale in $y$.

\section{Oblique modes: $\alpha R \gg 1$}

The oblique mode with the largest transient growth has an initial condition of the form

$$
\begin{gathered}
v_{0} \sim \alpha(\alpha R)^{-1 / 3} \tilde{v}(y) \exp \left(\mathrm{i} U(\alpha R)^{1 / 3} \tilde{t}_{0}\right), \\
\eta_{0} \sim \tilde{\eta}(y) \exp \left(\mathrm{i} U(\alpha R)^{1 / 3} \tilde{t}_{0}\right) .
\end{gathered}
$$

On a timescale of $\alpha^{-1}(\alpha R)^{1 / 3}$ this mode varies on a lengthscale of $(\alpha R)^{-1 / 3}$ in $y$. The norm of the solution stays order one for times of order $\alpha^{-1}(\alpha R)^{1 / 3}$, then suddenly (over an $O\left(\alpha^{-1}\right)$ time) $v$ and $\eta$ grow to be of order $\alpha(\alpha R)^{1 / 3}$ and $(\alpha R)^{1 / 3}$ respectively, ceasing to oscillate rapidly in $y$ at the same time. Just as suddenly, $v$ returns to being 
of order $\alpha(\alpha R)^{-1 / 3}$, but $\eta$ stays of order $(\alpha R)^{1 / 3}$, decaying slowly over a timescale $\alpha^{-1}(\alpha R)^{1 / 3}$, and oscillating rapidly in $y$ on a lengthscale $(\alpha R)^{-1 / 3}$ again.

In fact the transient growth we have found for $\alpha R \gg 1$ matches smoothly into that for $\alpha R \sim 1$ for small $\alpha$. The timescale for the growth is $\alpha^{-1}(\alpha R)^{1 / 3}$, and the maximum growth of the norm is $\alpha^{-1}(\alpha R)^{1 / 3}$. The maximum growth occurs for $\alpha=O(1 / R)$, as we mentioned earlier. However, we will see that we must choose $\alpha=O(1)$ in order to maximize the generation of a streamwise mode through the nonlinear terms while an oblique mode is growing and decaying.

\section{Eigenvalues of the linearized equations}

Here we determine the position of the eigenvalues of the linearized equations asymptotically as $R \rightarrow \infty$. This will enable us to determine how large a streamwise streak has to be in order to significantly perturb the eigenvalues, and in particular to make an eigenvalue become unstable.

Since all the eigenvalues of the base flow we consider are stable, there has been little interest in their asymptotic approximation (in contrast to the analysis of the neutral curve for plane Poiseuille flow, on which there is a vast literature). The asymptotic analysis that has been performed usually solves the equation in terms of special functions (generalized Airy functions for plane Couette flow), formulates the characteristic equation, and then approximates for large values of the Reynolds number (Drazin \& Reid 1981). This approach makes it quite difficult to see where the scalings of the different classes of eigenvalues come from, and hard to see how these eigenvalues would be perturbed by perturbing the equations.

The approach we take here is rather to approximate the equation for large values of the Reynolds number, find approximate solutions, and then apply the boundary conditions to determine the eigenvalues. This makes it very easy to see how the eigenvalues will be affected by perturbations, but it does mean that we have to take careful account of Stokes phenomenon. Some of the results of this section we believe to be new (or at least presented in a new way), while others are very well known; however, in both cases the crucial question we will address is how large a streamwise streak has to be in order to significantly affect the calculation.

In a common notation, we define the eigenvalues through the relationship

$$
v^{\alpha \beta}(y, t)=\mathrm{e}^{-\mathrm{i} \alpha \lambda t} v^{\alpha \beta}(y),
$$

so that a positive imaginary part of an eigenvalue $\lambda$ corresponds to instability. We drop the superscript $\alpha \beta$ and the overbar without possibility of confusion to give

$$
\begin{gathered}
\mathrm{i} \lambda v_{y y}-\mathrm{i} \lambda k^{2} v-\mathrm{i} U\left(v_{y y}-k^{2} v\right)+\mathrm{i} U^{\prime \prime} v+\frac{1}{\alpha R}\left(v_{y y y y}-2 k^{2} v_{y y}+k^{4} v\right)=0, \\
-\mathrm{i} \lambda \eta+\mathrm{i} U \eta+\mathrm{i} \frac{\beta}{\alpha} U^{\prime} v-\frac{1}{\alpha R}\left(\eta_{y y}-k^{2} \eta\right)=0 .
\end{gathered}
$$

The eigenvalues of (5.1) are known as the Orr-Sommerfeld modes, while the additional eigenvalues of (5.2) which have $v=0$ are known as the Squire modes. Early numerical computations of these eigenvalues were performed by Davey \& Drazin (1969). Note though that because of the extreme ill-conditioning of the problem (which is due to the extreme non-normality of the linearized operator) many of the early computations are not resolved.

A typical spectrum for plane Couette flow is shown in figure 9. Asymptotically the eigenvalues fall into two classes: those with imaginary component of order one, and 


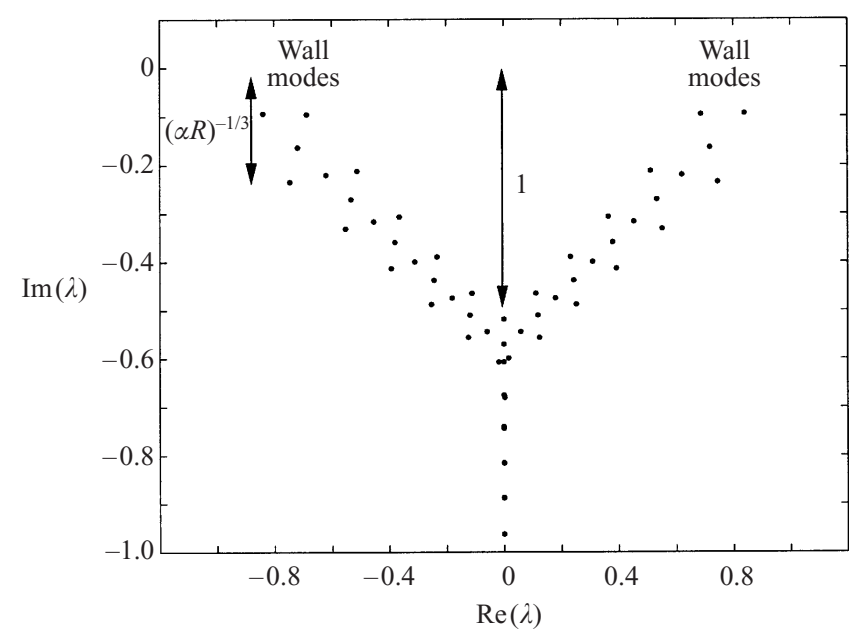

FiguRE 9. Eigenvalues of plane Couette flow with $\alpha=\beta=1$ and $R=2000$. The orders of magnitude of the imaginary parts as $R \rightarrow \infty$ are indicated. The eigenvalues were calculated in MATLAB using the Chebyshev hybrid spectral discretization described in Reddy \& Henningson (1993) and Reddy et al. (1993).

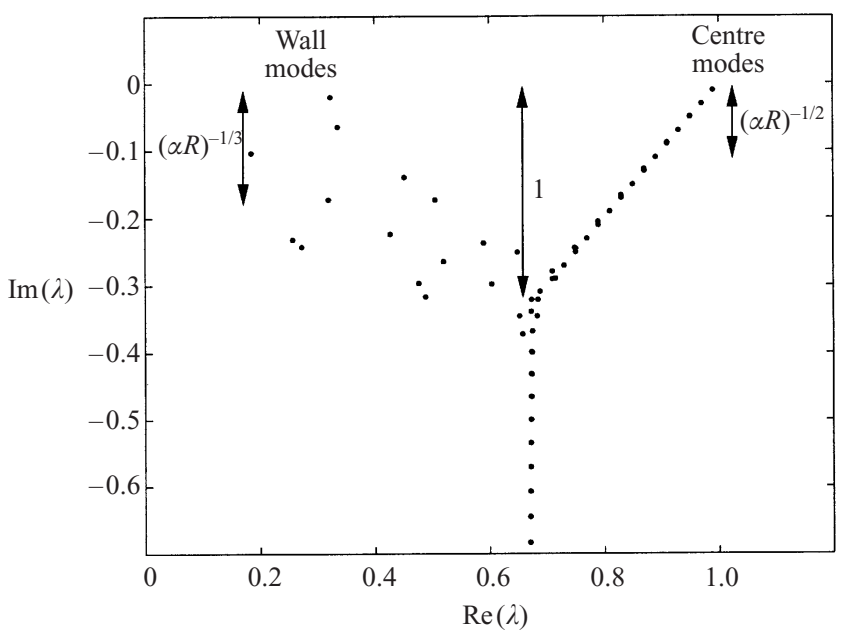

Figure 10. Eigenvalues of plane Poiseuille flow with $\alpha=\beta=1$ and $R=5000$. The orders of magnitude of the imaginary parts as $R \rightarrow \infty$ are indicated.

those with imaginary component of order $(\alpha R)^{-1 / 3}$ (which are localized in a region of order $(\alpha R)^{-1 / 3}$ near the boundary and are known as wall modes), as indicated in figure 9.

A typical spectrum for plane Poiseuille flow is shown in figure 10. Asymptotically there are now three families of modes, having eigenvalues with imaginary component $O(1), O\left((\alpha R)^{-1 / 3}\right)$ and $O\left((\alpha R)^{-1 / 2}\right)$ respectively. The modes with eigenvalues with imaginary part $O\left((\alpha R)^{-1 / 3}\right)$ are wall modes concentrated in a region of order $(\alpha R)^{-1 / 3}$ from the boundary, as in plane Couette flow. The new modes with eigenvalues having imaginary component $O\left((\alpha R)^{-1 / 2}\right)$ are 'centre modes'; these are localized in a region of order $(\alpha R)^{-1 / 4}$ around the centre of the channel.

For plane Poiseuille flow the even and odd modes decouple and may be considered 
independently if necessary, with the boundary conditions at either $y=1$ or $y=-1$ being replaced with $v=v^{\prime \prime}=\eta^{\prime}=0$ or $v^{\prime}=v^{\prime \prime \prime}=\eta=0$ at $y=0$ for even and odd modes respectively.

For $R>R_{c} \approx 5772.2$ one of the even wall modes in plane Poiseuille flow has a positive imaginary part (the Tollmien-Schlichting wave, $\alpha_{c} \approx 1.021, \beta_{c}=0$ ) corresponding to linear instability. However, we will find that the centre modes are the modes which exhibit the secondary instability involved in the routes to transition (i) and (ii) discussed in the Introduction.

Let us first show how the position of the eigenvalues can be determined asymptotically from (5.1)-(5.2), in the limit $\alpha R \rightarrow \infty$; we will then consider how large a streamwise streak has to be for the nonlinear terms on the right-hand side of (3.23)-(3.24) to affect this calculation.

We start with the eigenvalues which have order-one imaginary component. These are the most difficult to approximate, but they have received the least attention in the literature since they are all strongly stable. The following analysis is essentially an interpretation and extension of that by Morawetz (1952).

Of the eigenvalues which have order-one imaginary component, the Squire modes are the easiest to deal with, so we will examine them first.

\subsection{Squire eigenvalues with order-one imaginary component}

Although for plane Couette flow we can solve the equation exactly in terms of Airy functions and then approximate the characteristic equations, we prefer to approximate the solutions of the equation directly, since this makes the effect of a perturbation more transparent. With $v=0$ the asymptotic solutions of (5.2) as $\alpha R \rightarrow \infty$ are of WKв form. Setting

$$
\eta=B \exp \left((\alpha R)^{1 / 2} \phi\right),
$$

we find that at leading order

$$
-\mathrm{i} \lambda B+\mathrm{i} U B-\phi_{y}^{2} B=0,
$$

so that

$$
\phi= \pm \mathrm{e}^{\mathrm{i} \pi / 4} \int(U-\lambda)^{1 / 2} \mathrm{~d} y .
$$

At next order we find

$$
2 \phi_{y} B_{y}+\phi_{y y} B=0,
$$

so that

$$
B=\frac{1}{\phi_{y}^{1 / 2}} .
$$

We will show that the leading-order position of the eigenvalues is determined purely by the phase $\phi$ of these WKB solutions. In the following discussion the boundary $y=-1$ should be replaced by $y=0$ if we are dealing with plane Poiseuille rather than plane Couette flow.

If the solution of (5.5) is such that the real part of $\phi$ has a turning point in the interval $(-1,1)$ (as we will see that it is) then the eigenmode will be approximately equal to the WKB solution for which the real part of $\phi$ has a maximum (call this phase $\phi$ and the WKB approximation $\eta_{1}$ ), which is exponentially small at the boundaries by comparison to its value in the interior. Let us start with this solution and consider how to add multiples of the other solution (with phase $-\phi$; call this WKB approximation 


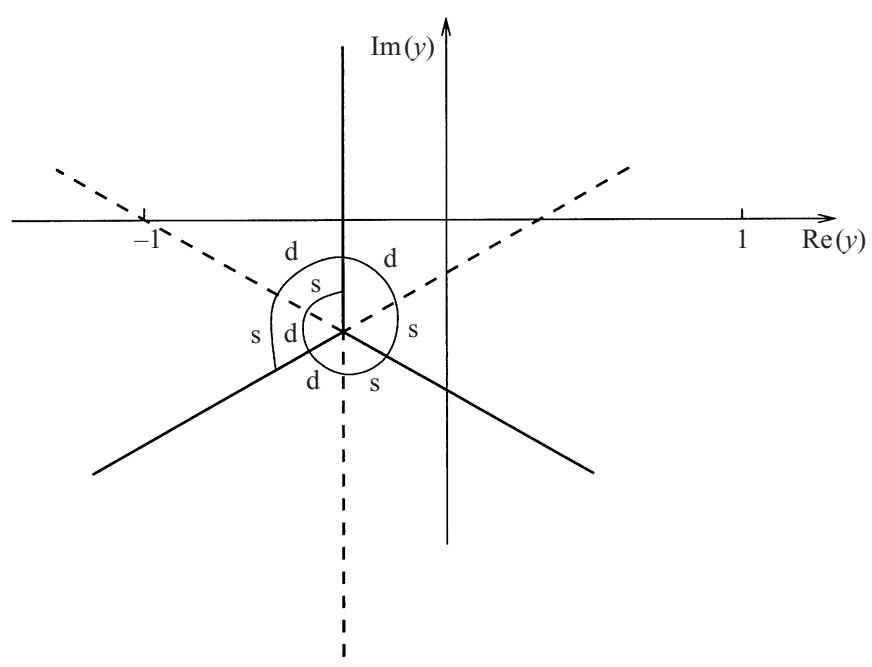

FIGURE 11. The Stokes line structure of $\eta$ for an eigenvalue on the upper left-hand branch of the ' $Y$ ' in figure 14. The spiral indicates which of the two WKB solutions are present in each sector (and represents the Riemann surface associated with the square-root branch point in the phase); $\mathrm{d}$ and $\mathrm{s}$ indicate the dominant and subdominant WKB approximations respectively. The subdominant WKB approximation is 'switched off' across the Stokes lines (solid), while the dominance of the two WKB approximations switches across the anti-Stokes lines (dashed).

$\eta_{2}$ ) in order to satisfy the boundary conditions (if there is no turning point in the real part of $\phi$ in the interval $(-1,1)$ then we may start with either wKB solution).

Suppose first that $\operatorname{Re}(\phi(-1))>\operatorname{Re}(\phi(1))$, so that $\eta_{1}(-1)$ exponentially dominates $\eta_{1}(1)$. Then we can ignore the boundary condition at $y=1$, but we must add an $O\left(\exp \left(2(\alpha R)^{1 / 2} \phi(-1)\right)\right)$ multiple of $\eta_{2}$ in order to satisfy the boundary condition at $y=-1$. However, this additional multiple of $\eta_{2}$ will exponentially dominate $\eta_{1}$ at $y=1$, since its phase there is $(\alpha R)^{1 / 2}(2 \phi(-1)-\phi(1))$ which has real part greater than that of $\phi(1)$. The only way out of this conundrum is that $\eta_{2}$ is switched off via Stokes phenomenon as we cross a Stokes line somewhere between $y=-1$ and $y=+1$.

Now, locally near the turning points in the complex plane where $\phi_{y}=0, \phi$ has a three-halves power singularity and the Stokes line structure is that of the Airy equation. We require the solution which has only a single subdominant wKB solution $\eta_{1}$ in the phase sector containing $y=1$, which is shown schematically in figure 11 . As we cross the Stokes line $\operatorname{Im}(\phi)=0$ the other wKB approximation $\eta_{2}$ is turned on, and is present to help us satisfy the boundary condition at $y=-1$. However, to do so it must have grown to be exactly the same size as $\eta_{1}$ there, i.e. $y=-1$ must lie on the anti-Stokes line (in agreement with the results of Morawetz 1952).

If $\operatorname{Re}(\phi(-1))>\operatorname{Re}(\phi(1))$ this gives eigenvalues lying on the upper left-hand branch of the ' $\mathrm{Y}$ ' in figure 14. If $\operatorname{Re}(\phi(1))>\operatorname{Re}(\phi(-1))$ then the structure of the Stokes lines is as shown schematically in figure 12, which gives eigenvalues lying on the upper right-hand branch of the $\mathrm{Y}$ in figure 14 .

The final case to consider is the possibility that $\operatorname{Re}(\phi(-1))=\operatorname{Re}(\phi(1))$. In this case an exponentially large multiple of the solution shown in figure $13(b)$ can be added to the solution shown in figure 13(a) to satisfy the boundary conditions at both $y= \pm 1$. This is possible so long as the right-hand anti-Stokes line in figure 13(a) does not cross $-1<y<1$, and gives the stem of the ' $\mathrm{Y}$ ' in figure 14 . 


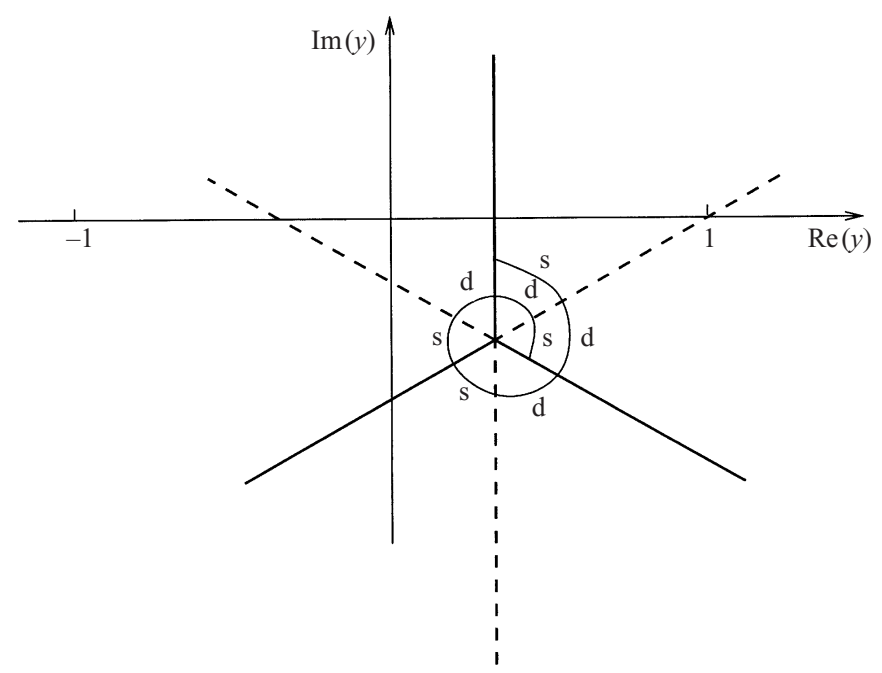

FIGURE 12. As figure 11 but for an eigenvalue on the upper right-hand branch of the ' $\mathrm{Y}$ ' in figure 14 .

Thus we can determine the asymptotic position of the eigenvalues in plane Couette and plane Poiseuille flow as follows.

\subsubsection{Plane Couette flow}

For plane Couette flow

$$
\phi=-\mathrm{e}^{\mathrm{i} \pi / 4} \frac{2(y-\lambda)^{3 / 2}}{3} .
$$

For $\operatorname{Re}(\lambda)<0$, the condition that $y=-1$ lies on the anti-Stokes line is

$$
\operatorname{Re}\left(\mathrm{e}^{\mathrm{i} \pi / 4} \frac{2(-1-\lambda)^{3 / 2}}{3}\right)=0
$$

i.e. $\arg (\lambda+1)=-\pi / 6$. For $\operatorname{Re}(\lambda)>0$, the condition that $y=+1$ lies on the anti-Stokes line is

$$
\operatorname{Re}\left(\mathrm{e}^{\mathrm{i} \pi / 4} \frac{2(1-\lambda)^{3 / 2}}{3}\right)=0,
$$

i.e. $\arg (\lambda-1)=-5 \pi / 6$. Finally, the condition that $\operatorname{Re}(\phi(1))=\operatorname{Re}(\phi(-1))$ is $\operatorname{Re}(\lambda)=0$.

In figure 14 we show the asymptotic lines that the Squire eigenvalues must lie on as $R \rightarrow \infty$, along with the Squire eigenvalues for $R=2000, \alpha=\beta=1$. If we were interested in the exact position of the eigenvalues it is a simple matter to determine these using the WKB solutions. However for our present purposes the lines are enough.

\subsubsection{Plane Poiseuille flow}

For plane Poiseuille flow

$$
\phi=\frac{\mathrm{e}^{-\mathrm{i} \pi / 4}}{2}\left(y\left(\lambda-1+y^{2}\right)^{1 / 2}+(\lambda-1) \log \left(\frac{y+\left(\lambda-1+y^{2}\right)^{1 / 2}}{(1-\lambda)^{1 / 2}}\right)\right) .
$$



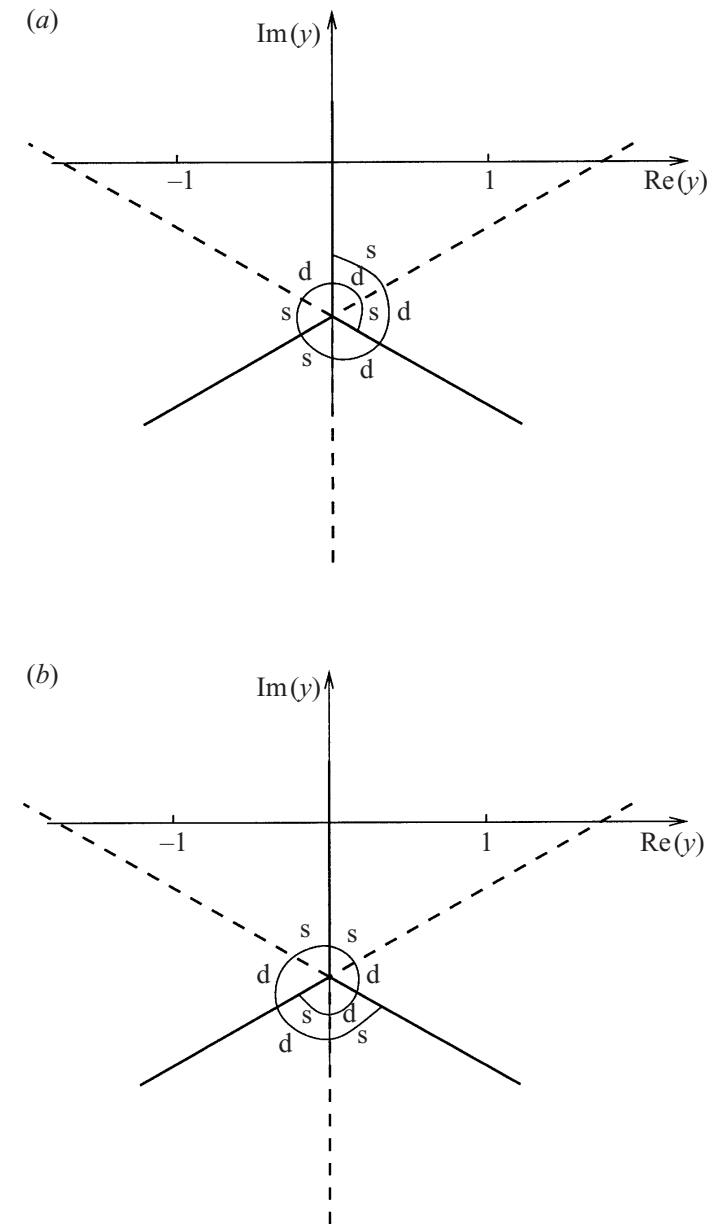

FiguRE 13. Stokes line structure of $\eta$ for eigenvalues lying on the stem of the ' $\mathrm{Y}$ ' in figure 14 . An exponentially large multiple of $(b)$ is added to $(a)$ in order to satisfy the boundary conditions at both $y= \pm 1$. This is possible so long as the anti-Stokes lines (dashed) do not cross $-1<y<1$, i.e. so long as $\operatorname{Im}(\lambda)$ is sufficiently large and negative.

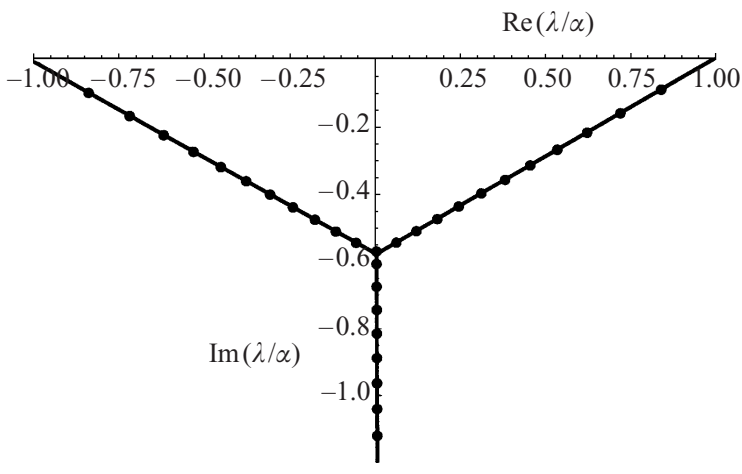

FIGURE 14. Asymptotic position of the Squire eigenvalues for plane Couette flow. The points are the numerical solution for the eigenvalues for $R=2000$ and $\alpha=\beta=1$. 


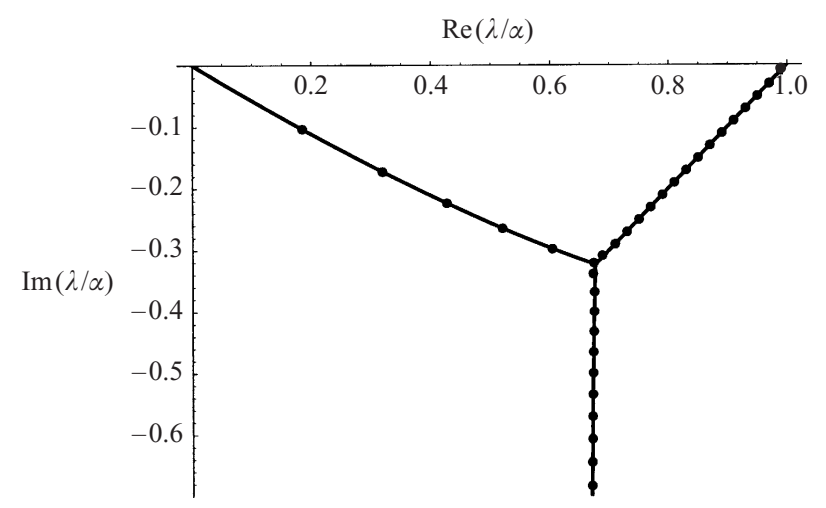

FIGURE 15. Asymptotic position of the Squire eigenvalues for plane Poiseuille flow. The points are the numerical solution for the eigenvalues for $R=5000$ and $\alpha=\beta=1$.

The condition that the anti-Stokes line goes through $y=0$ is that $\operatorname{Re}(\phi(0))=0$, i.e.

$$
\operatorname{Re}\left(\mathrm{e}^{-\mathrm{i} \pi / 4}(\lambda-1) \frac{\mathrm{i} \pi}{2}\right)=0
$$

i.e. $\arg (\lambda-1)=-3 \pi / 4$. The condition that the anti-Stokes line goes through $y=1$ is that $\operatorname{Re}(\phi(1))=0$, i.e.

$$
\operatorname{Re}\left(\mathrm{e}^{-\mathrm{i} \pi / 4}\left(\lambda^{1 / 2}+(\lambda-1) \log \left(\frac{1+\lambda^{1 / 2}}{(1-\lambda)^{1 / 2}}\right)\right)\right)=0 .
$$

Finally, the condition that $\operatorname{Re}(\phi(0))=\operatorname{Re}(\phi(1))$ gives

$$
\operatorname{Re}\left(\mathrm{e}^{-\mathrm{i} \pi / 4}\left(\lambda^{1 / 2}+(\lambda-1) \log \left(\frac{1+\lambda^{1 / 2}}{(1-\lambda)^{1 / 2}}\right)\right)\right)=\operatorname{Re}\left(\mathrm{e}^{-\mathrm{i} \pi / 4}(\lambda-1) \frac{\mathrm{i} \pi}{2}\right) .
$$

To ensure the anti-Stokes lines do not cross $0<y<1$ we are restricted to the portion of the curve lying below its intersection with the others. Note that, unlike plane Couette flow, the stem of the $\mathrm{Y}$ is not vertical.

In figure 15 we show the asymptotic lines that the Squire eigenvalues must lie on as $R \rightarrow \infty$, along with the Squire eigenvalues for $R=5000, \alpha=\beta=1$. Note that the odd and even modes are interspersed on the right-hand and lower branches, but are almost identical on the left-hand branch. Indeed they cannot be distinguished graphically; each point in figure 15 represents both an even and an odd mode. This is because the odd and even modes satisfy different boundary conditions at $y=0$, but the same boundary conditions at $y=1$.

\subsubsection{Perturbing the eigenvalues}

We can now determine how large a streamwise streak, $\eta^{0 \beta_{1}}$ say, has to be in order to perturb the Squire eigenvalues with imaginary component of order one. Equation (3.24) becomes

$$
L_{2}^{\alpha \beta}\left(\eta^{\alpha \beta}, v^{\alpha \beta}\right)=\sum_{\beta_{1}+\beta_{2}=\beta} N_{2}^{0 \beta_{1} \alpha \beta_{2}}\left(v^{0 \beta_{1}}, \eta^{0 \beta_{1}}, v^{\alpha \beta_{2}}, \eta^{\alpha \beta_{2}}\right),
$$

where the right-hand side is linear in $v^{\alpha \beta_{2}}, \eta^{\alpha \beta_{2}}$. Since the unperturbed eigenvalues all lie on the anti-Stokes line of the two WKB solutions, they will only be perturbed at leading order if the perturbation added on the right-hand side of (5.8) changes the 
phase $\phi$ of the WKB solutions, that is, the terms on the right-hand side of (5.8) have to be big enough to appear in equation (5.4). Now the left-hand side of equation (5.8) is $O(\alpha \eta)$, while the right-hand side is $O\left(\eta^{0 \beta_{1}} \alpha \eta\right)$. Hence the phase of the wKB solutions will be modified only if $\eta^{0 \beta_{1}}=O(1)$. Note that when this happens the equations (5.8) are all coupled in the crosswise direction $z$, even though they still decouple in the streamwise direction $x$. Thus the instability generated is a three-dimensional instability of a now three-dimensional base flow (in contrast to the three-dimensional instability of a two-dimensional base flow considered by Orszag \& Patera 1983).

Let us now determine the position of the Orr-Sommerfeld eigenvalues with orderone imaginary component.

\subsection{Orr-Sommerfeld eigenvalues with order-one imaginary component}

The asymptotic solutions of (5.1) comprise two inviscid solutions and two WKB solutions. To leading order the inviscid solutions satisfy

$$
\begin{gathered}
\mathrm{i} \lambda\left(v_{y y}-k^{2} v\right)-\mathrm{i} U\left(v_{y y}-k^{2} v\right)+\mathrm{i} U^{\prime \prime} v=0, \\
-\mathrm{i} \lambda \eta+\mathrm{i} U \eta+\mathrm{i} \frac{\beta}{\alpha} U^{\prime} v=0,
\end{gathered}
$$

while, setting

$$
v=A \exp \left((\alpha R)^{1 / 2} \phi\right), \quad \eta=B \exp \left((\alpha R)^{1 / 2} \phi\right),
$$

we find that at leading order the WKB solutions satisfy

$$
\begin{gathered}
\mathrm{i} \lambda \phi_{y}^{2}-\mathrm{i} U \phi_{y}^{2}+\phi_{y}^{4}=0, \\
-\mathrm{i} \lambda B+\mathrm{i} U B+\mathrm{i} \frac{\beta}{\alpha} U^{\prime} A-\phi_{y}^{2} B=0 .
\end{gathered}
$$

Hence the two WKB solutions again have

$$
\phi= \pm \mathrm{e}^{\mathrm{i} \pi / 4} \int(U-\lambda)^{1 / 2} \mathrm{~d} y,
$$

and we see that (5.13) implies that $A=0$, so that the relative scaling of $v$ and $\eta$ in (5.11) is wrong, and in fact we should have

$$
v=(\alpha R)^{-1 / 2} A \exp \left((\alpha R)^{1 / 2} \phi\right), \quad \eta=B \exp \left((\alpha R)^{1 / 2} \phi\right) .
$$

At next order we find

so that

$$
\begin{gathered}
2 A_{y} \phi_{y}+5 A \phi_{y y}=0, \\
2 \phi_{y} B_{y}+\phi_{y y} B=\mathrm{i} \frac{\beta}{\alpha} U^{\prime} A,
\end{gathered}
$$

$$
\begin{gathered}
A=\left(\phi_{y}\right)^{-5 / 2}, \\
B=\phi_{y}^{-1 / 2} \int \frac{\beta \mathrm{i} U^{\prime} A}{2 \alpha \phi_{y}^{1 / 2}} \mathrm{~d} y .
\end{gathered}
$$

We label the two WKB approximations, $v_{3}$ and $v_{4}$, such that

$$
\begin{gathered}
v_{3} \sim \phi_{y}^{-5 / 2} \exp \left((\alpha R)^{1 / 2} \phi\right), \\
v_{4} \sim \phi_{y}^{-5 / 2} \exp \left(-(\alpha R)^{1 / 2} \phi\right),
\end{gathered}
$$


where the sign of $\phi$ is chosen as before such that $\operatorname{Re}(\phi)>0$ where $\operatorname{Im}(\phi)=0$ with $y$ real, so that $v_{3}$ exponentially dominates $v_{4}$ there. We will label the two inviscid approximations, $v_{1}$ and $v_{2}$, shortly.

The position of the Orr-Sommerfeld eigenvalues is determined by Stokes phenomenon, as with the Squire modes. However, in this case, since there are four linearly independent solutions of the equation rather than two, there are many more Stokes switchings which are possible, and we need to take account of each of them. Each of the two inviscid solutions may turn on a multiple of the subdominant WKB approximation across the Stokes $\operatorname{line} \operatorname{Im}(\phi)=0$, while the dominant WKB approximation may turn on multiples of the inviscid solutions, as well as a multiple of the subdominant WKB solution.

Since plane Couette flow turns out to be much simpler than plane Poiseuille flow, we treat the flows separately, starting with Couette.

\subsubsection{Plane Couette flow}

For plane Couette flow two independent inviscid solutions are $\mathrm{e}^{k y}$, and $\mathrm{e}^{-k y}$. In fact these are exact solutions to (5.1), so that there is no Stokes line switching associated with them.

We now build up an eigenfunction as follows. Consider first an eigenvalue in the left half-plane. Then $v_{4}(1)$ exponentially dominates $v_{4}(-1)$ and $v_{3}(1)$.

Therefore, in the vicinity of $y=1$, we may take a suitable linear combination of the two inviscid approximations and the WKB approximation $v_{4}$ to satisfy the two boundary conditions there. Since $v_{4}^{\prime}=O\left((\alpha R)^{1 / 2} v_{4}\right)$, to leading order the inviscid solutions will satisfy the Dirichlet boundary condition, and an exponentially small amount of $v_{4}$, of order $(\alpha R)^{-1 / 2} \exp \left(-(\alpha R)^{1 / 2} \operatorname{Re}(\phi(1))\right.$, will be added to satisfy the Neumann condition.

Now let us consider the boundary conditions at $y=-1$. We still have the freedom to add a multiple of the solution shown schematically in figure 11, which approximates to $v_{3}$ near $y=1$ and is therefore negligible there. We must adjust the coefficient of this solution and the eigenvalue $\lambda$ in order to satisfy the two boundary conditions at $y=-1$.

However, as well as the dominant WKB approximation $v_{3}$ switching on a multiple of the subdominant WKB approximation $v_{4}$ across the Stokes line $\arg (y-\lambda)=\pi / 2$, it also switches on multiples of the two inviscid solutions.

If we define the two inviscid solutions by their asymptotic behaviour as $y \rightarrow \lambda$, so that

$$
v_{1} \sim 1, \quad v_{2} \sim y-\lambda
$$

as $y \rightarrow \lambda$ then the Stokes multiplier of the first is $O\left((\alpha R)^{5 / 12}\right)$, while that of the second is $O\left((\alpha R)^{3 / 4}\right)$. This can be deduced easily using the techniques in Chapman et al. (1998). Thus, as we cross the Stokes line as we move from $y=1$ to $y=-1$ we find

$$
v_{3} \rightarrow v_{3}+a v_{4}+(\alpha R)^{3 / 4} b v_{2}+(\alpha R)^{5 / 12} c v_{1},
$$

where the constants $a, b$ and $c$ are order one. It is possible to calculate the constants $a, b$ and $c$, but we leave them unspecified since their exact value is unimportant for our present purposes. Then, if at $y=+1$ our solution is approximated by

$$
\hat{A} v_{1}+\hat{B} v_{2}+v_{3}+\hat{C} v_{4}
$$

(where $v_{3}$ is negligible near $y=1$ and $\hat{C} v_{4}$ is negligible near $y=-1$ ) then at $y=-1$, 


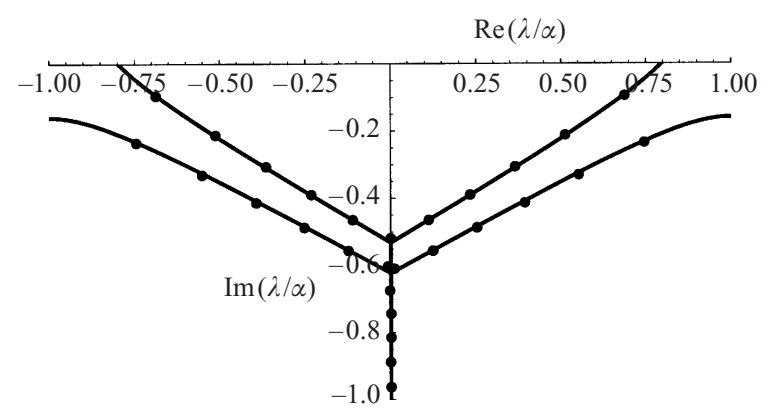

FIgURE 16. Asymptotic position of the Orr-Sommerfeld eigenvalues for plane Couette flow. The points are the numerical solution for the eigenvalues for $R=2000$ and $\alpha=\beta=1$.

having crossed the Stokes line, our solution is approximately

$$
\hat{A} v_{1}+\hat{B} v_{2}+\left(v_{3}+a v_{4}+(\alpha R)^{3 / 4} b v_{2}+(\alpha R)^{5 / 12} c v_{1}\right)+\hat{C} v_{4} .
$$

The boundary conditions at $y=-1$ give

$$
\hat{A} v_{1}+\hat{B} v_{2}+v_{3}+a v_{4}+(\alpha R)^{3 / 4} b v_{2} \sim 0,
$$

and

$$
\hat{A} v_{1}^{\prime}+\hat{B} v_{2}^{\prime}+(\alpha R)^{1 / 2} \phi^{\prime} v_{3}-a(\alpha R)^{1 / 2} \phi^{\prime} v_{4}+(\alpha R)^{3 / 4} b v_{2}^{\prime} \sim 0 .
$$

Since we already have one relation between $\hat{A}$ and $\hat{B}$ from the conditions at $y=1$, the only way we can satisfy the boundary conditions is if $\hat{A}$ and $\hat{B}$ are $O\left((\alpha R)^{3 / 4}\right)$, and one of $v_{3}$ or $v_{4}$ is $O\left((\alpha R)^{1 / 4}\right)$. Thus in this case we are not exactly on the anti-Stokes line, but very close to it. With $v_{3}=O\left((\alpha R)^{1 / 4}\right)$ we find $\operatorname{Re}(\phi)=(\alpha R)^{-1 / 2}(\log \alpha R) / 4$, while for $v_{4}=O\left((\alpha R)^{1 / 4}\right)$ we must have $\operatorname{Re}(\phi)=-(\alpha R)^{-1 / 2}(\log \alpha R) / 4$. Thus the eigenvalues lie on two lines, one either side of the anti-Stokes line, with a different wKB exponential dominant on each.

An alternative scenario is that the two WKB solutions are again of the same size at $y= \pm 1$, as with the Squire modes, so that there are also Orr-Sommerfeld modes on the stem of the ' $\mathrm{Y}$ '.

In figure 16 we show the curves $\operatorname{Re}(\phi)= \pm(\alpha R)^{-1 / 2}(\log \alpha R) / 4$, and $\operatorname{Re}(\phi(-1))=$ $\operatorname{Re}(\phi(1))$, along with the numerically determined Orr-Sommerfeld eigenvalues for $R=2000, \alpha=\beta=1$.

\subsubsection{Plane Poiseuille flow}

With plane Poiseuille flow the inviscid solutions are not so simple, and we have the added complication that these solutions themselves exhibit Stokes phenomenon, switching on a multiple of the subdominant WKB solution.

Let us define the two inviscid solutions, $v_{1}$ and $v_{2}$ say, through their asymptotic behaviour as $y \rightarrow \mathrm{i}(\lambda-1)$, so that

$$
\begin{gathered}
v_{1} \sim 1-\frac{\mathrm{i}}{(\lambda-1)^{1 / 2}}\left(y-\mathrm{i}(\lambda-1)^{1 / 2}\right) \log \left(y-\mathrm{i}(\lambda-1)^{1 / 2}\right), \\
v_{2} \sim y-\mathrm{i}(\lambda-1)^{1 / 2},
\end{gathered}
$$

as $y \rightarrow \mathrm{i}(\lambda-1)^{1 / 2}$. We label the two WKB solutions as before. Now as we cross the Stokes line $\operatorname{Im}(\phi)=0$, from left to right,

$$
\begin{aligned}
& v_{3} \rightarrow v_{3}+a v_{4}+b(\alpha R)^{3 / 4} v_{2}+c(\alpha R)^{5 / 12} v_{1}, \\
& v_{1} \rightarrow v_{1}+d(\alpha R)^{-3 / 4} v_{4},
\end{aligned}
$$


with $a, b, c$ and $d$ of order one, while there is no Stokes jump associated with $v_{2}$. Again, the techniques of Chapman et al. (1998) can be used to determine the size of the Stokes multiplier in $v_{1}$ easily.

Let us first examine the family of eigenvalues lying on the right-hand branch of the ' $\mathrm{Y}$ ' in figure 10, which become centre modes for small $\operatorname{Im}(\lambda)$. We start with a solution near $y=-1$ which is approximated by

$$
v \sim \hat{A} v_{1}+\hat{B} v_{2}+v_{3}+\hat{C} v_{4},
$$

where $\hat{C} v_{4}$ is exponentially negligible at $y=0$, while $v_{3}$ is exponentially negligible at $y=-1$. Then we choose $\hat{A}, \hat{B}$ and $\hat{C}$ to satisfy the two boundary conditions at $y=-1$. Since $v_{4}^{\prime}=O\left((\alpha R)^{1 / 2} v_{4}\right)$, to leading-order $\hat{A}$ and $\hat{B}$ are chosen to satisfy the Dirichlet boundary condition, with an exponentially small amount of $v_{4}$, of order $(\alpha R)^{-1 / 2} \exp \left(-(\alpha R)^{1 / 2} \operatorname{Re}(\phi(-1))\right.$, added to satisfy the Neumann condition.

Then, at $y=0$, having crossed the Stokes line, we have

$$
v \sim \hat{A} v_{1}+\hat{A} d(\alpha R)^{-3 / 4} v_{4}+\hat{B} v_{2}+v_{3}+a v_{4}+b(\alpha R)^{3 / 4} v_{2}+c(\alpha R)^{5 / 12} v_{1}+\hat{C} v_{4} .
$$

For the odd modes we need to satisfy $v=v^{\prime \prime}=0$ at $y=0$. Hence

$$
\begin{aligned}
\hat{A} v_{1}+\left(\hat{B}+b(\alpha R)^{3 / 4}\right) v_{2}+\left(\phi^{\prime}\right)^{-5 / 2} \mathrm{e}^{(\alpha R)^{1 / 2} \phi}+\left(a+\hat{A} d(\alpha R)^{-3 / 4}\right) \mathrm{e}^{-(\alpha R)^{1 / 2} \phi} \sim 0, & \\
\hat{A} v_{1}^{\prime \prime}+\left(\hat{B}+b(\alpha R)^{3 / 4}\right) v_{2}^{\prime \prime} & +\left(\phi^{\prime}\right)^{-1 / 2} \alpha R \mathrm{e}^{(\alpha R)^{1 / 2} \phi} \\
& +\left(a+\hat{A} d(\alpha R)^{-3 / 4}\right)\left(\phi^{\prime}\right)^{-1 / 2} \alpha R \mathrm{e}^{-(\alpha R)^{1 / 2} \phi} \sim 0 .
\end{aligned}
$$

Since we have already determined $\hat{A}$ to be the same order as $\hat{B}$, and since $b$ is $O(1)$, (5.24) implies that $\hat{A}$ and $\hat{B}$ are $O\left((\alpha R)^{3 / 4}\right)$. Then (5.25) is dominated by at least one of the WKB solutions, so that if there is to be a balance they must be of the same size, i.e. the eigenvalue must again lie on the anti-Stokes line.

For the even modes we have $v^{\prime}=v^{\prime \prime \prime}=0$ at $y=0$. Hence

$$
\begin{aligned}
& \hat{A} v_{1}^{\prime}+\left(\hat{B}+b(\alpha R)^{3 / 4}\right) v_{2}^{\prime}+(\alpha R)^{1 / 2}\left(\phi^{\prime}\right)^{-3 / 2} \mathrm{e}^{(\alpha R)^{1 / 2} \phi} \\
&-\left(a+\hat{A} d(\alpha R)^{-3 / 4}\right)(\alpha R)^{1 / 2}\left(\phi^{\prime}\right)^{-3 / 2} \mathrm{e}^{-(\alpha R)^{1 / 2} \phi} \sim 0, \\
& \hat{A} v_{1}^{\prime \prime \prime}+\left(\hat{B}+b(\alpha R)^{3 / 4}\right) v_{2}^{\prime \prime \prime}+\left(\phi^{\prime}\right)^{1 / 2}(\alpha R)^{3 / 2} \mathrm{e}^{(\alpha R)^{1 / 2} \phi} \\
&-\left(a+\hat{A} d(\alpha R)^{-3 / 4}\right)\left(\phi^{\prime}\right)^{1 / 2}(\alpha R)^{3 / 2} \mathrm{e}^{-(\alpha R)^{1 / 2} \phi} \sim 0 .
\end{aligned}
$$

Again, since we have already determined $\hat{A}$ and $\hat{B}$ to be the same order, and since $b$ is $O(1),(5.26)$ implies that $\hat{A}$ and $\hat{B}$ are $O\left((\alpha R)^{3 / 4}\right)$. Then, as before, (5.27) is dominated by at least one of the WKB solutions, and implies that the eigenvalue must lie on the anti-Stokes line.

Let us now examine the family of eigenvalues lying on the left-hand branch of the ' $\mathrm{Y}$ ' in figure 10, which become wall modes for small $\operatorname{Im}(\lambda)$. We start with a solution near $y=0$ which is approximated by

$$
v \sim \hat{A} v_{1}+\hat{B} v_{2}+v_{3}+\hat{C} v_{4},
$$

where $\hat{C} v_{4}$ is exponentially negligible at $y=-1$, while $v_{3}$ is exponentially negligible at $y=0$. Then we choose $\hat{A}, \hat{B}$ and $\hat{C}$ to satisfy the two boundary conditions at $y=0$. Since $v_{4}^{\prime}=O\left((\alpha R)^{1 / 2} v_{4}\right)$, to leading order $\hat{A}$ and $\hat{B}$ are chosen to satisfy boundary conditions containing derivatives of lowest order, with an exponentially small amount of $v_{4}$, added to satisfy the other condition. 


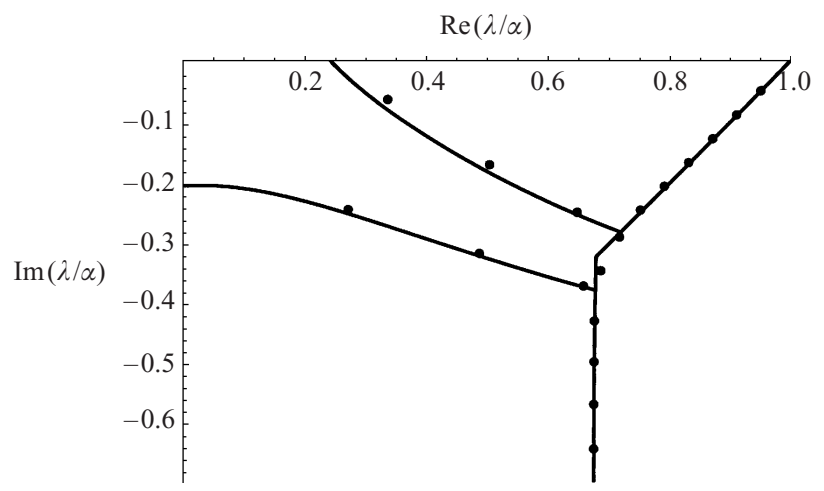

FIGURE 17. Asymptotic position of the odd Orr-Sommerfeld eigenvalues for plane Poiseuille flow. The points are the numerical solution for the eigenvalues for $R=5000$ and $\alpha=\beta=1$.

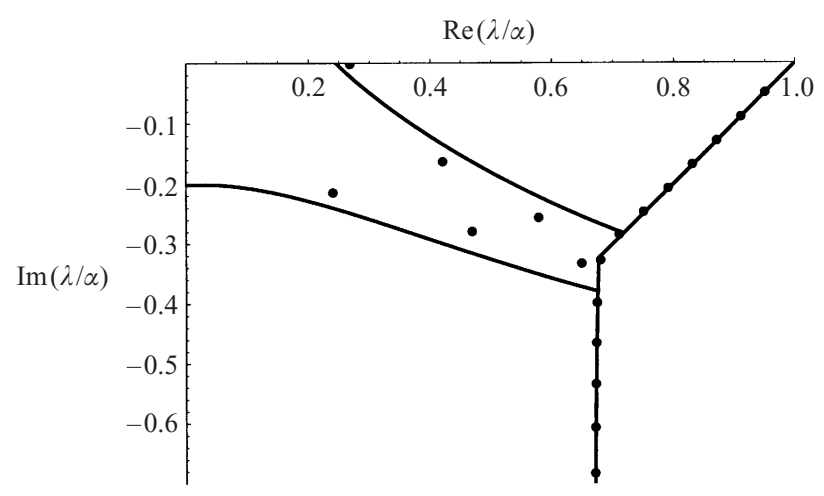

Figure 18. As figure 17 but for the even Orr-Sommerfeld eigenvalues for plane Poiseuille flow.

Then, at $y=-1$, having crossed the Stokes line, we have

$$
v \sim \hat{A}\left(v_{1}-d(\alpha R)^{-3 / 4} v_{4}\right)+\hat{B} v_{2}+v_{3}-a v_{4}-b(\alpha R)^{3 / 4} v_{2}-c(\alpha R)^{5 / 12} v_{1}+\hat{C} v_{4} .
$$

Hence the boundary conditions at $y=-1$ give

$$
\begin{aligned}
& \hat{A} v_{1}+\left(\hat{B}-b(\alpha R)^{3 / 4}\right) v_{2}+\left(\phi^{\prime}\right)^{-5 / 2} \mathrm{e}^{(\alpha R)^{1 / 2} \phi} \\
&-\left(a+\hat{A} d(\alpha R)^{-3 / 4}\right)\left(\phi^{\prime}\right)^{-5 / 2} \mathrm{e}^{-(\alpha R)^{1 / 2} \phi} \sim 0, \\
& \hat{A} v_{1}^{\prime}+\left(\hat{B}-b(\alpha R)^{3 / 4}\right) v_{2}^{\prime}+\left(\phi^{\prime}\right)^{-3 / 2}(\alpha R)^{1 / 2} \mathrm{e}^{(\alpha R)^{1 / 2} \phi} \\
&+\left(a+\hat{A} d(\alpha R)^{-3 / 4}\right)\left(\phi^{\prime}\right)^{-3 / 2}(\alpha R)^{1 / 2} \mathrm{e}^{-(\alpha R)^{1 / 2} \phi} \sim 0 .
\end{aligned}
$$

Since $\hat{A}$ and $\hat{B}$ are the same order, we must have $\hat{A}$ and $\hat{B}$ of order $(\alpha R)^{3 / 4}$. Then, as for plane Couette flow, the only way to satisfy the boundary conditions is for $\mathrm{e}^{ \pm(\alpha R)^{1 / 2} \phi}=O\left((\alpha R)^{1 / 4}\right)$, i.e. one of $v_{3}$ or $v_{4}$ must be $O\left((\alpha R)^{1 / 4}\right)$. Thus again we are close to the anti-Stokes line, with $\operatorname{Re}(\phi)= \pm(\alpha R)^{-1 / 2}(\log (\alpha R)) / 4$.

Finally, for the stem modes, $v_{3}$ and $v_{4}$ are the same size at $y=0$ and $y=-1$, so that asymptotically these eigenvalues lie on the same line as the the Squire modes.

In figures 17 and 18 we show the lines $\operatorname{Re}(\phi)= \pm(\alpha R)^{-1 / 2}(\log \alpha R) / 4$, and $\operatorname{Re}(\phi(-1))=\operatorname{Re}(\phi(0))$, along with the numerically determined Orr-Sommerfeld eigenvalues for $R=5000, \alpha=\beta=1$. 
Note that the agreement between the asymptotic lines and the numerical value of the eigenvalues is poor for the even Orr-Sommerfeld eigenvalues lying on the left-hand branches of the ' $\mathrm{Y}$ '. The reason for this is that when $\hat{A}$ and $\hat{B}$ are chosen to satisfy the leading-order behaviour in (5.28), namely

then

$$
\hat{A} v_{1}+\left(\hat{B}-b(\alpha R)^{3 / 4}\right) v_{2} \sim 0,
$$

$$
\hat{A} v_{1}^{\prime}+\left(\hat{B}-b(\alpha R)^{3 / 4}\right) v_{2}^{\prime}
$$

is close to zero, so that the eigenvalues are pushed closer to the anti-Stokes line. We show why this is so in Appendix B.

The eigenvalues on the right-hand branch of the ' $Y$ ' are almost equal for the odd and even modes. This has been commented on before Drazin \& Reid (1981), but as yet remains unexplained. The present analysis can be developed to explain this coincidence; we defer this discussion to Appendix C.

Having determined the asymptotic position of the Orr-Sommerfeld eigenvalues with order-one imaginary component, we can now determine the size of streamwise streak, $\eta^{0 \beta_{1}}$, necessary to significantly perturb these eigenvalues.

\subsubsection{Perturbing the eigenvalues}

After the growth of a streamwise streak, equation (3.23) becomes

$$
L_{1}^{\alpha \beta}\left(\eta^{\alpha \beta}, v^{\alpha \beta}\right)=\sum_{\beta_{1}+\beta_{2}=\beta} N_{1}^{0 \beta_{1} \alpha \beta_{2}}\left(v^{0 \beta_{1}}, \eta^{0 \beta_{1}}, v^{\alpha \beta_{2}}, \eta^{\alpha \beta_{2}}\right) \text {, }
$$

where the right-hand side is linear in $v^{\alpha \beta_{2}}, \eta^{\alpha \beta_{2}}$. Now, the key fact to emerge from the preceding analysis is that, as with the Squire modes, to leading order the eigenvalues all lie on the anti-Stokes lines associated with the WKB solutions, and therefore their position depends only on the phase of these solutions, $\phi$. Hence the eigenvalues will be perturbed at leading order only if the terms on the right-hand side of (5.30) are big enough to appear in equation (5.12).

Now, under the WKB approximation, the left-hand side of equation (5.30) is $O\left(\alpha^{2} R v\right)$, while the right-hand side is $O\left(\eta^{0 \beta_{1}} \alpha^{2} R v\right)$ (since $\eta=O\left((\alpha R)^{1 / 2} v / \alpha\right)$ ). Hence the phase of the WKB solutions will be modified when $\eta^{0 \beta_{1}}=O(1)$.

As with the Squire modes, once the streak is big enough to perturb the eigenvalues the equations (5.30) are all coupled in the crosswise direction $z$, even though they still decouple in the streamwise direction $x$, so that the base flow is three-dimensional.

Thus far we have dealt with all the eigenvalues with order-one imaginary component. The eigenvalues with small imaginary component must be treated separately, and because of their slow decay rate have received more attention in the literature. For those with the anti-Stokes line passing through $y= \pm 1$ all the action takes place near the boundary, so that they are known as wall modes, while for those with the anti-Stokes line passing through $y=0$ (for plane Poiseuille flow) all the action takes place near the centreline, and they are known as centre modes.

\subsection{Wall modes in plane Couette flow}

For the wall modes near $y=-1$ (for example) the correct scaling is $y=-1+$ $\alpha^{-1 / 3} R^{-1 / 3} \bar{y}, \lambda=-1+\alpha^{-1 / 3} R^{-1 / 3} \bar{\lambda}$ (see, for example Corcos \& Sellars 1959; Gill 1965), giving

$$
\begin{gathered}
\mathrm{i} \bar{\lambda} v_{\bar{y} \bar{y}}-\mathrm{i} \bar{y} v_{\bar{y} \bar{y}}+v_{\bar{y} \bar{y} \bar{y} \bar{y}}=0, \\
\mathrm{i} \bar{\lambda} \eta-\mathrm{i} \bar{y} \eta+\eta_{\bar{y} \bar{y}}=\frac{\mathrm{i}(\alpha R)^{1 / 3} \beta v}{\alpha} .
\end{gathered}
$$


Now, the outer inviscid solutions can be determined as before, and satisfy the Dirichlet condition at $y=1$ to leading order. Hence the normalised outer solution away from the wall is

As $y \rightarrow-1$ this gives

$$
v=\sinh (y+1)
$$

$$
v \sim-\sinh 2+R^{-1 / 3} \bar{y} \cosh 2+\cdots .
$$

Hence the boundary conditions on (5.31)-(5.32) are

$$
\begin{gathered}
v=v_{\bar{y}}=\eta=0 \quad \text { on } \quad \bar{y}=0, \\
v \rightarrow-\sinh 2, \quad \eta \rightarrow 0 \quad \text { as } \quad \bar{y} \rightarrow \infty .
\end{gathered}
$$

The sinh 2 here simply determines the relative scaling of the eigenfunction in the outer inviscid and inner wall regions; all that is important is that this factor is non-zero. Equation (5.31) can easily be solved in terms of generalized Airy functions, and (5.32) in terms of Airy functions, to give expressions for the eigenvalues. However, for our present purposes all that is important is the scaling.

\subsubsection{Perturbation of the wall-mode eigenvalues in Couette flow}

Let us now determine how large a streamwise streak has to be to perturb the wall-mode eigenvalues. There are two ways that this can be performed: we can either modify equations (5.31) and (5.32), or we can modify the boundary conditions (5.33) and (5.34). To modify the boundary conditions, we need to modify the inviscid solutions. For these the left-hand side of (3.23) is $O(\alpha v)$, while the right-hand side is $O\left(\eta^{0 \beta_{1}} \alpha^{2} \eta\right)$. Since $v=O(\alpha \eta)$ for the inviscid solutions, these will be modified when $\eta^{0 \beta_{1}}$ is $O(1)$.

Now in the inner region near the wall the left-hand side of (3.23) is $O\left(\alpha(\alpha R)^{1 / 3} v\right)$, while the right-hand side is $O\left(\eta^{0 \beta_{1}} \alpha^{2}(\alpha R)^{1 / 3} \eta\right)$. Since $v=O\left(\alpha(\alpha R)^{-1 / 3} \eta\right)$ in the wall region, this means the right-hand side will be in the leading-order balance when $\eta^{0 \beta_{1}}$ is $O\left((\alpha R)^{-1 / 3}\right)$. However, we must remember that $\eta^{0 \beta_{1}}$ varies on an order-one lengthscale in $y$, and must vanish at the boundary, so that in the inner wall region it takes values order $(\alpha R)^{-1 / 3}$ smaller than in the bulk of the flow. Thus to achieve a value of $\eta^{0 \beta_{1}}$ of order $(\alpha R)^{-1 / 3}$ in the wall region requires a streamwise streak of order one in the bulk.

For the Squire wall modes, the left-hand side of (3.24) is $O\left(\alpha(\alpha R)^{-1 / 3} \eta\right)$, while the right-hand side is $O\left(\eta^{0 \beta_{1}} \alpha \eta\right)$. Again, this means that $\eta^{0 \beta_{1}}$ must be $O\left((\alpha R)^{-1 / 3}\right)$ in the wall region, and therefore $O(1)$ in the bulk of the flow.

\subsection{Wall modes in plane Poiseuille flow}

For the wall modes near $y=-1$ (for example) the correct scaling is (see, for example Corcos \& Sellars 1959; Gill 1965), $y=-1+\alpha^{-1 / 3} R^{-1 / 3} \bar{y}, \lambda=\alpha^{-1 / 3} R^{-1 / 3} \bar{\lambda}$, giving

$$
\begin{gathered}
\mathrm{i} \bar{\lambda} v_{y y}-2 \mathrm{i} \bar{y} v_{y y}+v_{y y y y}=0, \\
\mathrm{i} \bar{\lambda} \eta-2 \mathrm{i} \bar{y} \eta+\eta_{y y}=\frac{\mathrm{i}(\alpha R)^{1 / 3} \beta v}{\alpha} .
\end{gathered}
$$

Now in this case the outer inviscid solutions satisfy

$$
\begin{gathered}
\left(1-y^{2}\right)\left(v_{y y}-k^{2} v\right)+2 v=0, \\
\alpha\left(1-y^{2}\right) \eta=2 \beta y v
\end{gathered}
$$


to leading order. Now, as $y \rightarrow-1$,

$$
v \sim c_{1}(1+(y+1) \log (y+1))+c_{2}\left((1+y)+\frac{(1+y)^{2}}{2}+\cdots\right),
$$

for some $c_{1}$ and $c_{2}$. Hence, providing $c_{1} \neq 0$, the boundary conditions on (5.35)-(5.36) are

$$
\begin{array}{cll}
v=v_{\bar{y}}=\eta=0 & \text { on } & \bar{y}=0, \\
v \rightarrow c_{1}, \quad \eta \rightarrow 0 & \text { as } & \bar{y} \rightarrow \infty .
\end{array}
$$

Hence, for $c_{1}$ of order one, the eigenvalues are the same as for plane Couette flow (once the wall shear has been normalised); in particular they are all stable, and a bulk streamwise streak of order one is required to perturb them at leading order.

The difference between the stability characteristics of plane Couette flow and plane Poiseuille flow arises because for small $\alpha$ and $\beta$ it turns out that $c_{1} \sim 0$ for the even modes. There is a vast amount of literature on the neutral stability curve for plane Poiseuille flow, and we do not delve into it here, since it is peripheral for our purposes (but refer the reader to the extensive discussion in Drazin \& Reid 1981). However, we do indicate very briefly how the scalings arise from the present analysis.

5.4.1. Small $\alpha$ and $\beta$; lower branch of the marginal stability curve

When $\alpha \rightarrow 0$ (but $\alpha R \gg 1$ ), and with $\beta=0$, the outer inviscid solutions satisfy

$$
\left(1-y^{2}\right) v_{y y}+2 v=0,
$$

at leading order in $\alpha$, with even solution

$$
v=1-y^{2}
$$

and odd solution

$$
v=y+\left(1-y^{2}\right) \tanh ^{-1} y .
$$

Now, for the even solution, $c_{1}=0$ and

$$
v \sim 2(1+y)-(1+y)^{2}
$$

as $y \rightarrow-1$. The boundary conditions on (5.35)-(5.36) then depend on the relative scaling of $R$ and $\alpha$. For the even modes the first-order correction term to (5.40) satisfies

$$
\left(1-y^{2}\right) v_{y y}+2 v+\alpha^{2}\left(1-y^{2}\right)^{2}-2 \lambda=0,
$$

with even solution

$$
v=\frac{\alpha^{2}}{30}\left(y^{2}\left(3 y^{2}-11\right)+4\left(1-y^{2}\right) \log \left(1-y^{2}\right)\right)+\lambda .
$$

Hence as $y \rightarrow-1$,

$$
v \sim 2(1+y)+\lambda-\frac{4 \alpha^{2}}{15}+\cdots .
$$

The canonical scaling for $\alpha$ so that both these terms appear in the boundary conditions on the inner eigenvalue problem is such that in the inner scaling $1+y=O\left(\alpha^{2}\right)$, i.e. $\alpha=O\left(R^{-1 / 7}\right)$. Note that $\lambda$ is then also of the same order as $\alpha^{2}$. The boundary conditions on the inner problem (5.35)-(5.36) are then

$$
\begin{aligned}
v=v_{\bar{y}}=\eta=0 \quad \text { on } \quad \bar{y}=0, & \\
v \sim 2 \bar{y}+\bar{\lambda}-\frac{4\left(\alpha^{7} R\right)^{1 / 3}}{15}, & \eta \rightarrow 0 \quad \text { as } \quad \bar{y} \rightarrow \infty .
\end{aligned}
$$


However, when we apply these boundary conditions we find that there is an unstable eigenvalue for all $\alpha$ greater than a critical value. Thus we have determined the lower branch of the marginal stability curve, and there is another scaling between $\alpha=O\left(R^{-1 / 7}\right)$ and $\alpha=O(1)$ which will give the upper branch.

\subsubsection{Upper branch of the marginal stability curve}

We can see from (5.42) that there is another scaling possible. Suppose that, to leading order, $\lambda=4 \alpha^{2} / 15$. Then the constant term in $v$ as $y \rightarrow-1$ is $O\left(\alpha^{4}\right)$ and a different scaling is necessary for this term to appear in the boundary conditions of the inner problem. To have both the linear and constant terms appearing in the boundary conditions we require that in the inner scaling $1+y=O\left(\alpha^{4}\right)$. However, since $\lambda=O\left(\alpha^{2}\right)$ the inner scaling no longer has $y$ and $\lambda$ of the same order, and the inner problem is slightly different. In the inner region we balance $\lambda v_{y y}$ with $(\alpha R)^{-1} v_{y y y y}$ so that the scaling is $1+y \sim(\lambda \alpha R)^{-1 / 2}$, giving $\alpha=O\left(R^{-1 / 11}\right)$. Note that then the inner region is $1+y=O\left(R^{-4 / 11}\right)=O\left((\alpha R)^{-2 / 5}\right)$, while $\lambda=O\left(R^{-2 / 11}\right)=O\left((\alpha R)^{-1 / 5}\right)$. This means the maximum of the WKB solutions lies away from the boundary layer, and the matching conditions on the inner region are more complicated.

\subsection{Centre modes in plane Poiseuille flow}

The final set of eigenmodes we need to consider are the centre modes of plane Poiseuille flow, which are localized near $y=0$. The correct scaling is $y=R^{-1 / 4} \alpha^{-1 / 4} \bar{y}$, $\lambda=1+\alpha^{-1 / 2} R^{-1 / 2} \bar{\lambda}$ (see, for example Corcos \& Sellars 1959; Gill 1965), giving

$$
\begin{gathered}
\mathrm{i} \bar{\lambda} v_{\bar{y} \bar{y}}+\mathrm{i} \bar{y}^{2} v_{\bar{y} \bar{y}}-2 \mathrm{i} v+v_{\bar{y} \bar{y} \bar{y}}=0, \\
-\mathrm{i} \bar{\lambda} \eta-\mathrm{i} \bar{y}^{2} \eta-\eta_{\bar{y} \bar{y}}=\frac{2 \mathrm{i} \beta(\alpha R)^{1 / 4} \bar{y} v}{\alpha} .
\end{gathered}
$$

The outer inviscid solutions satisfy

with solution

$$
y^{2}\left(v_{y y}-k^{2} v\right)-2 v=0,
$$

$$
v=C_{1}\left(\frac{\cosh (k y)}{y}-k \sinh (k y)\right)+C_{2}\left(\frac{\sinh (k y)}{y}-k \cosh (k y)\right) .
$$

Since this must satisfy the Dirichlet condition at $y=-1$ to leading order we have

Now as $y \rightarrow 0$,

$$
v=\frac{k(1+y)}{y} \cosh (k y)-\frac{1+k^{2} y}{y} \sinh (k y) .
$$

$$
v \sim \frac{k \cosh k-\sinh k}{y}
$$

Hence, so long as $k \neq 0$, the boundary conditions on (5.43)-(5.44) are

$$
\begin{gathered}
v=v_{\bar{y} \bar{y}}=\eta_{\bar{y}}=0 \quad \text { on } \quad \bar{y}=0, \\
v \rightarrow \frac{k \cosh k-\sinh k}{\bar{y}}, \quad \eta \rightarrow 0 \quad \text { as } \quad \bar{y} \rightarrow-\infty,
\end{gathered}
$$

for odd modes and

$$
\begin{gathered}
v_{\bar{y}}=v_{\bar{y} \bar{y} \bar{y}}=\eta=0 \quad \text { on } \quad \bar{y}=0, \\
v \rightarrow \frac{k \cosh k-\sinh k}{\bar{y}}, \quad \eta \rightarrow 0 \quad \text { as } \quad \bar{y} \rightarrow-\infty,
\end{gathered}
$$

for even modes. 
This time, as $k \rightarrow 0$, the leading-order inviscid solutions are

$$
v=\frac{C_{1}}{y}+C_{2} y^{2},
$$

so that satisfying the Dirichlet condition at $y=-1$ is

$$
v=\frac{1+y^{3}}{y} .
$$

Then, as $y \rightarrow 0$,

$$
v \sim \frac{1}{y},
$$

so that there is nothing special about $k \rightarrow 0$ for the centre modes.

\subsubsection{Perturbation of the centre-mode eigenvalues for plane Poiseuille flow}

Let us now determine how large a streamwise streak has to be to perturb the centre-mode eigenvalues at leading order.

As for the wall modes, to modify the centre modes we can either modify equation (5.43), or we can modify the boundary conditions, which come from the inviscid solution on the outer region. We have already seen that the inviscid solutions will be modified when $\eta^{0 \beta_{1}}$ is of order one.

In the inner region the left-hand side of (3.23) is of order $\alpha v$, while the righthand side is of order $\eta^{0 \beta_{1}} \alpha(\alpha R)^{1 / 2} v$. Hence the equation for the centre modes will be perturbed at leading order when the streamwise streak $\eta^{0 \beta_{1}}$ is $O\left((\alpha R)^{-1 / 2}\right)$ in the centre region.

Now, because in plane Poiseuille flow the odd and even modes decouple, if the streamwise streak is an odd mode ( $v^{0 \beta_{1}}$ odd, $\eta^{0 \beta_{1}}$ even) then $\eta^{0 \beta_{1}}$ takes values near the origin of the same order as in the bulk, and $\eta^{0 \beta_{1}}$ of order $(\alpha R)^{-1 / 2}$ in the bulk will perturb the eigenvalues at leading order. However, if the streamwise streak is an even mode ( $v^{0 \beta_{1}}$ even, $\eta^{0 \beta_{1}}$ odd) then $\eta^{0 \beta_{1}}$ is of order $(\alpha R)^{-1 / 4}$ smaller near the origin than in the bulk, and it requires an $\eta^{0 \beta_{1}}$ of order $(\alpha R)^{-1 / 4}$ in the bulk to perturb the eigenvalues at leading order.

Thus to minimize the perturbation required we should consider modes with $\alpha$ as large as possible. The approximation of the eigenvalues in $\S 5.5$ is valid so long as $\alpha \ll R^{1 / 3}$, at which point additional terms appear in the leading-order balance. Above this value of $\alpha$ the left-hand side of the equation grows more rapidly than the right, and the perturbation becomes weaker again. Thus the canonical scaling would seem to be $\alpha=O\left(R^{1 / 3}\right)$, for which modes an odd streamwise streak of $O\left(R^{-2 / 3}\right)$ or an even streamwise streak of $O\left(R^{-1 / 3}\right)$ will perturb the eigenvalues at leading order. However, we will see in the next section that even though these large-wavenumber eigenvalues are perturbed at leading order they remain stable, and that the order-one wavenumber modes are the ones which become unstable. With $\alpha$ of order one it requires an odd streamwise streak of order $R^{-1 / 2}$ to generate instability.

Note that, since $\eta^{0 \beta_{1}} \ll 1$, none of the wall modes, including the TollmienSchlichting mode, are perturbed at leading order, so that the neutral stability curve of this mode is unchanged.

\subsection{Summary}

In this section we have shown how to determine the position of the eigenvalues of the linearized Navier-Stokes equations asymptotically as $\alpha R \rightarrow \infty$. We found 
that to leading order the eigenvalues with $O(1)$ imaginary component all lie on the anti-Stokes lines of the two WKB solutions. Thus to perturb these eigenvalues by an order-one amount, which will be necessary if they are to be perturbed to have positive imaginary component, it is necessary to change the phase of the wKB solutions, rather than their amplitude, i.e. the perturbation must be sufficiently strong to have an effect on equation (5.4) or (5.12). We found that such a perturbation requires a streamwise streak $\eta^{0 \beta_{1}}$ of order one, i.e. the base flow must be perturbed at leading order.

For the eigenvalues which have small imaginary component, the wall modes and (for plane Poiseuille flow) the centre modes, a different approach is necessary, and the eigenvalues are determined by a local 'inner region' in the vicinity of the wall or centre respectively. To perturb these eigenvalues into instability it is necessary for the perturbation to be sufficient to change this local equation (or its boundary conditions).

The wall modes have eigenvalues with imaginary component of order $(\alpha R)^{-1 / 3}$, and also require a streamwise streak $\eta^{0 \beta_{1}}$ of order one to perturb them at leading order.

The centre modes have eigenvalues with imaginary component of order $(\alpha R)^{-1 / 2}$ (so long as $\alpha \leqslant O\left(R^{1 / 3}\right)$ ). An odd streamwise streak of order $(\alpha R)^{-1 / 2}$ will perturb these eigenvalues, while if the streamwise streak is even it must be $O\left((\alpha R)^{-1 / 4}\right)$. The most sensitive modes therefore correspond to $\alpha=O\left(R^{1 / 3}\right)$, for which an odd streamwise streak of order $R^{-2 / 3}$ or an even streamwise streak of order $R^{-1 / 3}$ is enough to perturb the eigenvalues at leading order. However, even though these large-wavenumber eigenvalues are perturbed at leading order they remain stable, and it is in fact the order-one wavenumber modes which are the ones which become unstable. With $\alpha$ of order one it requires an odd streamwise streak of order $R^{-1 / 2}$, or an even streamwise streak of order $R^{-1 / 4}$ to generate instability.

We have briefly included the rather special scalings which give the one unstable mode of plane Poiseuille flow. These will not be important for our bypass route to transition (in fact, we will find that the centre modes are the important ones in plane Poiseuille flow), but are included to show how the present work fits in with traditional hydrodynamic stability theory.

The robustness of the eigenvalues of (5.1) and (5.2) with respect to perturbations is remarkable, given that, as discussed in the Introduction, the operators (3.17) and (3.18) are both highly non-normal as $R \rightarrow \infty$ (in fact the eigenvalue problems for these operators are exponentially ill-conditioned). Many values of $\lambda$ with real part between -1 and 1 fail to give eigenfunctions only by an exponentially small amount in $R$, since there is a corresponding solution which is exponentially small near each boundary by comparison to its maximum (Reddy \& Henningson 1993).

Finally we note that in the numerical simulations of Reddy et al. (1998) the secondary instability of a streamwise streak in Couette flow occurred through the perturbation of a mode with order-one imaginary component, whereas that in Poiseuille flow occurred through the perturbation of a centre mode, in agreement with the present asymptotic analysis.

\section{Secondary instability of centre modes}

Let us examine briefly the nature of the secondary instability of the centre modes. Since the Fourier modes in $z$ are now all coupled it is easier to return to the 
Navier-Stokes equations and linearise about the new base flow, which is

$$
\begin{aligned}
\boldsymbol{u} & =\left(1-y^{2}, 0,0\right)+\delta\left(u_{0}(y, z), 0,0\right)+\cdots, \\
\boldsymbol{\omega} & =(0,0,2 y)+\delta\left(0, \frac{\partial u_{0}}{\partial z}(y, z),-\frac{\partial u_{0}}{\partial y}(y, z)\right),
\end{aligned}
$$

where $\delta u_{0}(y, z)$ is the small transiently growing streamwise streak (with zero streamwise wavenumber). Let us first examine the eigenvalue problem for $\alpha$ large. Linearising the Navier-Stokes equations (3.5)-(3.11) about this flow gives

$$
\begin{gathered}
\xi_{t}-(\eta U)_{y}+u_{z} U^{\prime}-\zeta_{z} U-\frac{1}{R}\left(\xi_{x x}+\xi_{y y}+\xi_{z z}\right)=\delta\left(-\left(-\eta_{0} u-\eta u_{0}\right)_{y}+\left(\zeta_{0} u+\zeta u_{0}\right)_{z}\right) \\
\eta_{t}+\eta_{x} U+v_{z} U^{\prime}-\frac{1}{R}\left(\eta_{x x}+\eta_{y y}+\eta_{z z}\right)=\delta\left(\left(-\eta_{0} u-\eta u_{0}\right)_{x}-\left(\eta_{0} w-\zeta_{0} v\right)_{z}\right) \\
\zeta_{t}-u_{x} U^{\prime}+\zeta_{x} U-\left(v U^{\prime}\right)_{y}-\frac{1}{R}\left(\zeta_{x x}+\zeta_{y y}+\zeta_{z z}\right)=\delta\left(-\left(\zeta_{0} u+\zeta u_{0}\right)_{x}+\left(\eta_{0} w-\zeta_{0} v\right)_{y}\right) \\
u_{x}+v_{y}+w_{z}=0 \\
\xi=w_{y}-v_{z} \\
\eta=-w_{x}+u_{z} \\
\zeta=v_{x}-u_{y}
\end{gathered}
$$

with $(u, v, w)=\mathbf{0}$ on $y= \pm 1$, where

$$
\zeta_{0}=-\frac{\partial u_{0}}{\partial y}, \quad \eta_{0}=\frac{\partial u_{0}}{\partial z} .
$$

Subtracting the $x$-derivative of (6.5) from the $z$-derivative of (6.3) we find that the Orr-Sommerfeld equation is perturbed to

$$
\begin{aligned}
-\nabla^{2} v_{t}+\frac{1}{R} \nabla^{4} v-2 v_{x}-\left(1-y^{2}\right) & \nabla^{2} v_{x}=\delta\left[\zeta_{0} u_{x x}+u_{0} \zeta_{x x}-\left(\eta_{0} w_{x}\right)_{y}+\left(\zeta_{0} v_{x}\right)_{y}\right. \\
& \left.+\left(\eta_{0} u\right)_{y z}+\left(\eta u_{0}\right)_{y z}+\left(\zeta_{0} u\right)_{z z}+\left(\zeta u_{0}\right)_{z z}\right] .
\end{aligned}
$$

Writing $u=\hat{u}(y, z) \mathrm{e}^{-\mathrm{i} \alpha \lambda t+\mathrm{i} \alpha x}$ etc., with $\bar{u}_{0}$ even, using the centre-mode scalings

$$
\begin{gathered}
y=(\alpha R)^{-1 / 4} \bar{y}, \quad \lambda=1+(\alpha R)^{-1 / 2} \bar{\lambda}, \quad \hat{v}=\bar{v}, \quad \hat{u}=\alpha^{-1}(\alpha R)^{1 / 4} \bar{u}, \\
\hat{w}=\alpha^{-2}(\alpha R)^{1 / 4} \bar{w}, \quad \hat{\eta}=\alpha^{-1}(\alpha R)^{1 / 4} \bar{\eta}, \quad \hat{\xi}=\alpha^{-2}(\alpha R)^{1 / 2} \bar{\xi}, \quad \hat{\zeta}=\alpha^{-1}(\alpha R)^{1 / 2} \bar{\zeta},
\end{gathered}
$$

we find that (6.4) and (6.11) become (with $\alpha$ large)

$$
\begin{gathered}
-\mathrm{i} \bar{\lambda} \bar{\eta}-\mathrm{i} \bar{y}^{2} \bar{\eta}-2 \bar{y}_{z}-\bar{\eta}_{\bar{y} \bar{y}}+\epsilon^{2} \alpha^{2} \bar{\eta}-\epsilon^{2} \bar{\eta}_{z z}=\delta \epsilon^{-2}\left(-\mathrm{i} u_{0}(0, z) \bar{\eta}+\eta_{0}(0, z) \bar{v}_{\bar{y}}+\cdots\right), \\
\mathrm{i}\left(\bar{\lambda}+\bar{y}^{2}\right)\left(\bar{v}_{\bar{y} \bar{y}}-\epsilon^{2} \alpha^{2} \bar{v}+\epsilon^{2} v_{z z}\right)+\bar{v}_{\bar{y} \bar{y} \bar{y} \bar{y}}-2 \epsilon^{2} \alpha^{2} \bar{v}_{\bar{y} \bar{y}}+2 \epsilon^{2} v_{\bar{y} \bar{y} z z}-2 \epsilon^{4} \alpha^{2} v_{z z} \\
+\epsilon^{4} v_{z z z z}+\epsilon^{4} \alpha^{4} \bar{v}-2 \mathrm{i} \bar{v}=\mathrm{i} \delta \epsilon^{-2} u_{0}(0, z)\left(\bar{v}_{\bar{y} \bar{y}}-\epsilon^{2} \alpha^{2} \bar{v}+\epsilon^{2} v_{z z}\right)+\cdots,
\end{gathered}
$$

where $\epsilon=(\alpha R)^{-1 / 4}$. The terms involving $\epsilon \partial / \partial z$ have been retained since the linearly independent solutions which go to make up the eigenfunctions are of WKB form in $z$. Those involving $\epsilon \alpha$ have been retained since we wish to consider large $\alpha$.

Clearly the eigenvalues are perturbed by the right-hand side once $\delta$ is order $\epsilon^{2}=(\alpha R)^{-1 / 2}$ as predicted in the previous section. To minimize this we wish to make $\alpha$ as large as possible. From the left-hand side of (6.12) and (6.13) it is clear that the 
distinguished limit is $\alpha=O\left(\epsilon^{-1}\right)$ giving $\alpha=O\left(R^{1 / 3}\right)$, above which $\delta$ increases with increasing $\alpha$. This is the distinguished limit considered by Davey \& Nguyen (1971) for the case of pipe Poiseuille flow. However, in contrast to the results of Davey \& Nguyen (1971), with $\alpha=O\left(R^{1 / 3}\right)$ even though the eigenvalues of (6.12) and (6.13) are perturbed at leading order, they all remain in the lower half-plane and are therefore stable. To see this multiply (6.12) by $\bar{\eta}$ and (6.13) by $\bar{v}_{y y}-\epsilon^{2} \alpha^{2} \bar{v}+\epsilon^{2} \bar{v}_{z z}$ and integrate over $y$ and $z$ to give the Squire eigenvalues as

$$
\lambda=\frac{\int_{-\infty}^{0} \int_{0}^{l_{z}}\left[\left(\delta \epsilon^{-2} u_{0}(0, z)-y^{2}\right)|\eta|^{2}-\mathrm{i}\left(\left|\eta_{y}\right|^{2}+\epsilon^{2} \alpha^{2}|\eta|^{2}+\epsilon^{2}\left|\eta_{z}\right|^{2}\right)\right] \mathrm{d} z \mathrm{~d} y}{\int_{-\infty}^{0} \int_{0}^{l_{z}}|\eta|^{2} \mathrm{~d} z \mathrm{~d} y}
$$

and the Orr-Sommerfeld eigenvalues as

$$
\begin{aligned}
& \lambda=\frac{\int_{-\infty}^{0} \int_{0}^{l_{z}}\left[\left(\delta \epsilon^{-2} u_{0}(0, z)-y^{2}\right)\left|v_{y y}-\alpha^{2} \epsilon^{2} v+\epsilon^{2} v_{z z}\right|^{2}-2\left(\left|v_{y}\right|^{2}+\alpha^{2} \epsilon^{2}|v|^{2}+\epsilon^{2}\left|v_{z}\right|^{2}\right)\right] \mathrm{d} z \mathrm{~d} y}{\int_{-\infty}^{0} \int_{0}^{l_{z}}\left|v_{y y}-\alpha^{2} \epsilon^{2} v+\epsilon^{2} v_{z z}\right|^{2} \mathrm{~d} z \mathrm{~d} y} \\
& -\mathrm{i}\left[\frac{\int_{-\infty}^{0} \int_{0}^{l_{z}}\left[\left|v_{y y y}-\alpha^{2} \epsilon^{2} v_{y}+\epsilon^{2} v_{y z z}\right|^{2}+\epsilon^{2}\left|v_{y y z}-\alpha^{2} \epsilon^{2} v_{z}+\epsilon^{2} v_{z z z}\right|^{2}+\alpha^{2} \epsilon^{2}\left|v_{y y}-\alpha^{2} \epsilon^{2} v+\epsilon^{2} v_{z z}\right|^{2}\right] \mathrm{d} z \mathrm{~d} y}{\int_{-\infty}^{0} \int_{0}^{l_{z}}\left|v_{y y}-\alpha^{2} \epsilon^{2} v+\epsilon^{2} v_{z z}\right|^{2} \mathrm{~d} z \mathrm{~d} y}\right] .
\end{aligned}
$$

We can see why the large- $\alpha$ modes should be stable by considering the problem with $\alpha$ of order one, which gives

$$
\begin{gathered}
-\mathrm{i} \bar{\lambda} \bar{\xi}+\left(\bar{\eta} \bar{y}^{2}\right)_{\bar{y}}-2 \bar{u}_{z} \bar{y}+\bar{\zeta}_{z} \bar{y}^{2}-\left(\bar{\xi}_{\bar{y} \bar{y}}-\epsilon^{2} \alpha^{2} \bar{\xi}+\epsilon^{2} \bar{\xi}_{z z}\right)=\delta(\alpha R)^{1 / 2}\left(\eta_{0} \bar{u}_{\bar{y}}+\bar{\eta}_{\bar{y}} u_{0}+\left(\bar{\zeta} u_{0}\right)_{z}\right) \\
-\mathrm{i} \bar{\lambda} \bar{\eta}-\mathrm{i} \bar{\eta} \bar{y}^{2}-2 \bar{v}_{z} \bar{y}-\left(\bar{\eta}_{\bar{y} \bar{y}}-\epsilon^{2} \alpha^{2} \bar{\eta}+\epsilon^{2} \bar{\eta}_{z z}\right)=\delta(\alpha R)^{1 / 2}\left(-\mathrm{i} \eta_{0} \bar{u}-\mathrm{i} \bar{\eta} u_{0}-\alpha^{-2}\left(\eta_{0} \bar{w}\right)_{z}\right) \\
-\mathrm{i} \bar{\lambda} \bar{\zeta}+2 i \bar{u} \bar{y}-\mathrm{i} \bar{\zeta} \bar{y}^{2}+2(\bar{v} \bar{y})_{\bar{y}}-\left(\bar{\zeta}_{\bar{y} \bar{y}}-\epsilon^{2} \alpha^{2} \bar{\zeta}+\epsilon^{2} \bar{\zeta}_{z z}\right)=\delta(\alpha R)^{1 / 2}\left(-\mathrm{i} \bar{\zeta} u_{0}+\eta_{0} \alpha^{-2} \bar{w}_{\bar{y}}\right) \\
\mathrm{i} \bar{u}+\bar{v}_{\bar{y}}+\alpha^{-2} \bar{w}_{z}=0 \\
\bar{\xi}=\bar{w}_{\bar{y}}-\alpha^{2} \epsilon^{2} \bar{v}_{z} \\
\bar{\eta}=-\mathrm{i} \bar{w}+\bar{u}_{z} \\
\bar{\zeta}=\mathrm{i} \alpha^{2} \epsilon^{2} \bar{v}-\bar{u}_{\bar{y}} .
\end{gathered}
$$

The key term which is lost in the limit $\alpha \rightarrow \infty$ is the last term on the right of (6.15). The instability is essentially an inviscid instability due to an inflectional profile of $u_{0}$ in $z$, as has been observed in the numerical simulations of Reddy et al. (1998). To see this suppose that $\delta(\alpha R)^{1 / 2} \gg 1$. Then $\bar{\lambda}=O\left(\delta(\alpha R)^{1 / 2}\right)$ and the solution is independent of $y$ to leading order and satisfies

$$
\begin{gathered}
-\mathrm{i} \bar{\lambda} \bar{\xi}-\epsilon^{2} \bar{\xi}_{z z}+\epsilon^{2} \alpha^{2 \bar{\xi}} \sim \delta(\alpha R)^{1 / 2}\left(\bar{\zeta} u_{0}\right)_{z} \\
-\mathrm{i} \bar{\lambda} \bar{\eta}-\epsilon^{2} \bar{\eta}_{z z}+\epsilon^{2} \alpha^{2} \bar{\eta} \sim \delta(\alpha R)^{1 / 2}\left(-\mathrm{i} \eta_{0} \bar{u}-\mathrm{i} \bar{\eta} u_{0}-\alpha^{-2}\left(\eta_{0} \bar{w}\right)_{z}\right), \\
-\mathrm{i} \bar{\lambda} \bar{\zeta}-\epsilon^{2} \bar{\zeta}_{z z}+\epsilon^{2} \alpha^{2 \bar{\zeta}} \sim \delta(\alpha R)^{1 / 2}\left(-\mathrm{i} \bar{\zeta} u_{0}\right), \\
\mathrm{i} \bar{u}+\alpha^{-2} \bar{w}_{z} \sim 0, \\
\bar{\xi} \sim-\alpha^{2} \epsilon^{2} \bar{v}_{z},
\end{gathered}
$$


giving

$$
\begin{gathered}
\bar{\eta} \sim-\mathrm{i} \bar{w}+\bar{u}_{z}, \\
\bar{\zeta} \sim \mathrm{i} \alpha^{2} \epsilon^{2} \bar{v},
\end{gathered}
$$

$$
-\bar{\lambda}\left(\alpha^{2} \bar{w}-\bar{w}_{z z}\right)-i \epsilon^{2}\left(\bar{w}_{z z z z}-\alpha^{2} \bar{w}_{z z}+\alpha^{4} \bar{w}\right) \sim \delta(\alpha R)^{1 / 2}\left(-\left(\alpha^{2} \bar{w}-\bar{w}_{z z}\right) u_{0}-\frac{\partial^{2} u_{0}}{\partial z^{2}} \bar{w}\right),
$$

which is just the Orr-Sommerfeld problem for the stability of the crosswise velocity profile $u_{0}(0, z)$, as we would expect. For certain choices of $u_{0}(0, z)$ (and we can choose $u_{0}(0, z)$ arbitrarily by combining different Fourier modes in $z$ in the analysis of $\S 4)$ there exists an inviscid instability for wavenumbers $\alpha$ less than some critical wavenumber $\alpha_{c}$ (in particular $u_{0}(0, z)$ must have an inflection point) (see e.g. Drazin $\&$ Reid 1981).

The critical wavenumber $\alpha_{c}$ is related to the typical lengthscale for variation of $u_{0}(0, z)$. To obtain instability with $\alpha$ of order $R^{1 / 3}$ we would need $u_{0}(0, z)$ to vary on the lengthscale $R^{-1 / 3}$. This requires crosswise wavenumbers $\beta$ of order $R^{1 / 3}$, for which the transient growth is weaker. Large $\beta$ requires a wKB solution to (4.6)-(4.7), which still results in growth of $\eta$ to be $O(R v)$, but now from (3.19) $u$ is only order $R^{-1 / 3} \eta$. This factor of $R^{1 / 3}$ outweighs the factor $R^{-1 / 6}$ we gain in $\delta$ from having $\alpha$ of order $R^{1 / 3}$, so that the optimal route to transition is to have $\alpha$ and $\beta$ of order one.

Since in the limit $\delta(\alpha R)^{1 / 2} \rightarrow \infty$ for some $\alpha$ of order one there is an unstable eigenvalue, by continuity there will continue to be an unstable eigenvalue so long as $\delta(\alpha R)^{1 / 2}$ is greater than some threshold (independent of $R$ ). Thus the threshold value of $\delta$ to generate instability is $O\left(R^{-1 / 2}\right)$.

Reddy et al. (1998) calculate the decay rate of the least stable centre mode numerically and find it to be proportional to $R^{-0.55}$ in the range of Reynolds numbers they consider (as opposed to the asymptotic value $R^{-1 / 2}$ ). Instead of the mathematical argument about eigenvalues above, they then use a physical argument by balancing this dissipation with the growth rate of the inflectional instability (which is proportional to $\delta$ ), concluding that a streak of size $O\left(R^{-0.55}\right)$ will induce instability (which leads them to a threshold amplitude for a streamwise vortex of $O\left(R^{-1.55}\right)$ as we will see in the next section).

\section{Threshold amplitudes for transition}

We are now in a position to combine the results of $\S \S 4$ and 5 to determine the threshold amplitudes for transition for flows with periodic boundary conditions.

The two modes to transition described in the Introduction and demonstrated in the toy model of $\S 2$ are

(i) streamwise vortices $\rightarrow$ streamwise streaks

$\rightarrow$ secondary instability of oblique modes,

(ii) oblique modes $\rightarrow$ streamwise vortices $\rightarrow$ streamwise streaks

$\rightarrow$ secondary instability of oblique modes.

Let us consider plane Couette and plane Poiseuille flow separately.

\subsection{Plane Couette Flow}

7.1.1. Route (i): an initial perturbation in the form of streamwise vortices

Consider an initial condition in the form of a streamwise vortex, that is $\eta=0$, $v=v_{0}^{0 \beta_{1}}(y) \exp \left(\mathrm{i} \beta_{1} z\right)$ for some order-one $\beta_{1}$. We have seen in $\S 4$ that if $v_{0}^{0 \beta_{1}}(y)$ varies 
on an order-one lengthscale in $y$ then it will decay over a timescale $R$, while at the same time generating a streamwise streak $\eta^{0 \beta_{1}}$ of order $R v_{0}^{0 \beta_{1}}$. During this evolution the right-hand side of (3.25) and (3.26) remains negligible, so that $v^{0 \beta_{1}}$ and $\eta^{0 \beta_{1}}$ are evolving linearly.

Then, in $\S 5$, we saw that a streamwise streak of order one is necessary to perturb the eigenvalues of the oblique modes (3.27)-(3.28) at leading order. Thus the threshold amplitude for secondary instability in the oblique modes with an initial perturbation in the form of a streamwise vortex is $O\left(R^{-1}\right)$.

Note that we have found that all the eigenvalues of the oblique modes are perturbed relatively by the same order of magnitude when $\eta^{0 \beta_{1}}$ is of order one, so that there is no easy way to predict which eigenvalue is the first to cross the real axis. This will depend on the nature of the initial condition and the exact size of the perturbation to the eigenvalues (rather than just its order of magnitude). However, once the secondary instability has taken place, the growth rate is order $(\alpha R)^{-1 / 3}$ for the wall modes, but order one for the other modes. Hence we expect the secondary instability to occur predominantly away from the walls of the channel. Since the instability has an orderone growth rate, while the streamwise modes are decaying over a timescale $R$, in the limit of large $R$ the secondary instability will generate fully developed order-one oblique modes well before the streamwise modes have time to decay to zero, in which case we enter the fully nonlinear regime of the equations and we expect to have transition from the laminar state.

\subsubsection{Route (ii): an initial perturbation in the form of optimally growing oblique modes}

Now consider an initial condition in the form of optimally growing oblique modes, that is $\eta=0, v=v_{0}^{\alpha \beta}(y) \mathrm{e}^{\mathrm{i} \alpha x} \mathrm{e}^{\mathrm{i} \beta z}$ for some order-one $\alpha$ and $\beta$ which we will choose shortly.

We have seen in $\S 4$ which initial conditions $v_{0}^{\alpha \beta}$ will generate the maximum linear growth in the solution. For such initial conditions $v^{\alpha \beta}$ and $\eta^{\alpha \beta}$ oscillate on a lengthscale $(\alpha R)^{-1 / 3}$ (so that the norm of the initial condition $\left\|\boldsymbol{u}_{0}\right\|=O\left((\alpha R)^{1 / 3}\left\|v_{0}^{\alpha \beta}\right\|\right)$ ). For a time of order $\alpha^{-1}(\alpha R)^{1 / 3}$ they remain order $v_{0}^{\alpha \beta}$ and order $\alpha^{-1}(\alpha R)^{1 / 3} v_{0}^{\alpha \beta}$ respectively, when they suddenly (over an order-one time) grow to be order $(\alpha R)^{2 / 3} v_{0}^{\alpha \beta}$ and $\alpha^{-1}(\alpha R)^{2 / 3} v_{0}^{\alpha \beta}$ respectively, and cease to oscillate. After this, $v^{\alpha \beta}$ immediately (i.e. over an $O\left(\alpha^{-1}\right)$ time) returns to be order $v_{0}^{\alpha \beta}$, while $\eta^{\alpha \beta}$ remains of order $\alpha^{-1}(\alpha R)^{2 / 3} v_{0}^{\alpha \beta}$, decaying over the slow timescale $(\alpha R)^{1 / 3}$ and oscillating again on the lengthscale $(\alpha R)^{-1 / 3}$.

The final piece of information we need to calculate the threshold amplitude for transition by route (ii) is question (iii) of $\S 3$, namely the the size of the streamwise vortex produced by the nonlinear terms in (3.25) and (3.26) during the evolution of $v^{\alpha \beta}$ and $\eta^{\alpha \beta}$.

However, we also need to show that the nonlinear terms in (3.27) and (3.28) are of lower order than the linear terms during this first phase of the linear growth of the initial oblique mode (so that the evolution is indeed linear), which we do in Appendix E. Note that if this were not the case, then the alternative route to transition involving the 'boot-strapping' of oblique modes through nonlinear coupling, would have a lower threshold amplitude than route (ii).

Now for the oblique mode with the maximum transient growth, the phase of $v^{\alpha \beta}$ and $\eta^{\alpha \beta}$ is pure imaginary, while the nonlinear terms in (3.25) and (3.26) involve products of the modes with streamwise wavenumber $\alpha$ and $-\alpha$. Hence we see from (4.32), (4.33) that if the initial conditions are such that the blow-up times of these two 
modes are the same then the phases cancel in the product so that the nonlinear terms of (3.25) and (3.26) vary on an order-one lengthscale in $y$. On the other hand, if the blow-up times are different, then the nonlinear terms of (3.25) and (3.26) oscillate in $y$ on the lengthscale $(\alpha R)^{-1 / 3}$. The relevant scenario is the first one, since forcing (3.25) and (3.26) with a more and more rapidly varying inhomogeneous term leads to a WKB-type of solution which is of lower and lower amplitude (since the derivatives on the left-hand side of the equation become larger and larger) and which decays more and more rapidly. Note that if the phases of $v^{\alpha \beta}$ and $\eta^{\alpha \beta}$ were not pure imaginary then the products in (3.25) and (3.26) would be forced to be rapidly varying, so that the optimally growing oblique mode (which has a pure imaginary phase) also leads to the strongest possible forcing of streamwise modes. Thus the initial condition we should give is in fact $v=v_{0}^{\alpha \beta}(y) \mathrm{e}^{\mathrm{i} \alpha x} \mathrm{e}^{\mathrm{i} \beta z}+v_{0}^{-\alpha \beta}(y) \mathrm{e}^{-\mathrm{i} \alpha x} \mathrm{e}^{\mathrm{i} \beta z}$ for some non-zero $\alpha$ and $\beta$.

We have seen that the evolution of $v^{\alpha \beta}$ and $\eta^{\alpha \beta}$ proceeds through three phases; as we might expect, the dominant phase for the generation of streamwise vortices is the third phase, where $\eta^{\alpha \beta}$ is large, slowly decaying, and rapidly oscillating in $y$. Since $\eta^{\alpha \beta}=O\left(\alpha^{-1}(\alpha R)^{2 / 3} v_{0}\right), \eta_{y}^{\alpha \beta}=O\left(\alpha^{-1}(\alpha R) v_{0}\right)$, the terms $\eta^{\alpha \beta} \eta_{y}^{-\alpha \beta}$ and $\eta^{-\alpha \beta} \eta_{y}^{\alpha \beta}$ on the righthand side of (3.23) are $O\left(\alpha^{-2}(\alpha R)^{5 / 3} v_{0}^{2}\right)$. However, since the phases of $\eta^{\alpha \beta}$ and $\eta^{-\alpha \beta}$ are equal and opposite, the combination $\eta^{\alpha \beta} \eta_{y}^{-\alpha \beta}+\eta^{-\alpha \beta} \eta_{y}^{\alpha \beta}$ is only $O\left(\alpha^{-2}(\alpha R)^{4 / 3} v_{0}^{2}\right)$. Since it holds this value for a time of order $\alpha^{-1}(\alpha R)^{1 / 3}$, if $\alpha \leqslant O(1)$ the nonlinear terms in (3.23) will produce a non-oscillatory $v^{0 \beta}$ of order $\alpha^{-1}(\alpha R)^{5 / 3} v_{0}^{2}$ (which is $R\left\|\boldsymbol{u}_{0}\right\|^{2}$ ) during this phase, while if $\alpha \gg 1$ the nonlinear terms in (3.23) will produce a non-oscillatory $v^{0 \beta}$ of order $\alpha^{-5}(\alpha R)^{5 / 3} v_{0}^{2}$ (or $\alpha^{-4} R\left\|\boldsymbol{u}_{0}\right\|^{2}$ ). Hence to maximize the streamwise vortex produced we should choose $\alpha$ to be less than or equal to order one.

We are now in a position to determine the threshold amplitude for an initial disturbance in the form of oblique modes. We have already seen that a streamwise vortex $v^{0 \beta}$ of order $R^{-1}$ is enough to induce transition, and we have now seen that an initial oblique-mode perturbation $v_{0}$ of order $R^{-4 / 3}$ is enough to produce this. However, remember that this initial perturbation is oscillating rapidly on the lengthscale $R^{-1 / 3}$, so that both its $L^{2}$ and $L^{\infty}$ norm are in fact of order $R^{-1}$.

For completeness we should check that the oblique modes are not producing large streamwise streaks at the same time as they are producing the streamwise vortices. We find that the nonlinear terms in (3.26) are of order $R^{4 / 3} v_{0}^{2}$, which is smaller than the forcing term $v$ on the left-hand side, so that they are indeed negligible.

In summary, the threshold amplitude for transition with an initial perturbation both in the form of a streamwise vortex and in the form of an oblique mode is $O\left(R^{-1}\right)$.

In figure 19 we show a schematic diagram of the route to transition showing the norm of the solution at each stage.

\subsection{Plane Poiseuille flow}

Let us now determine the threshold amplitude for initial perturbations of plane Poiseuille flow. As explained in the introduction, we are concerned with the small domain of attraction of the stable modes of the system, and will ignore the unstable modes of non-periodic flows.

\subsubsection{Route (i): an initial perturbation in the form of streamwise vortices}

Consider an initial condition in the form of a streamwise vortex, that is $\eta=0$, $v=v_{0}^{0 \beta_{1}}(y) \exp \left(\mathrm{i} \beta_{1} z\right)$ for some non-zero $\beta_{1}$. As for plane Couette flow $v_{0}^{0 \beta_{1}}(y)$ will decay over a timescale $R$, while at the same time generating a streamwise streak $\eta^{0 \beta_{1}}$ of order $R v_{0}^{0 \beta_{1}}$. 


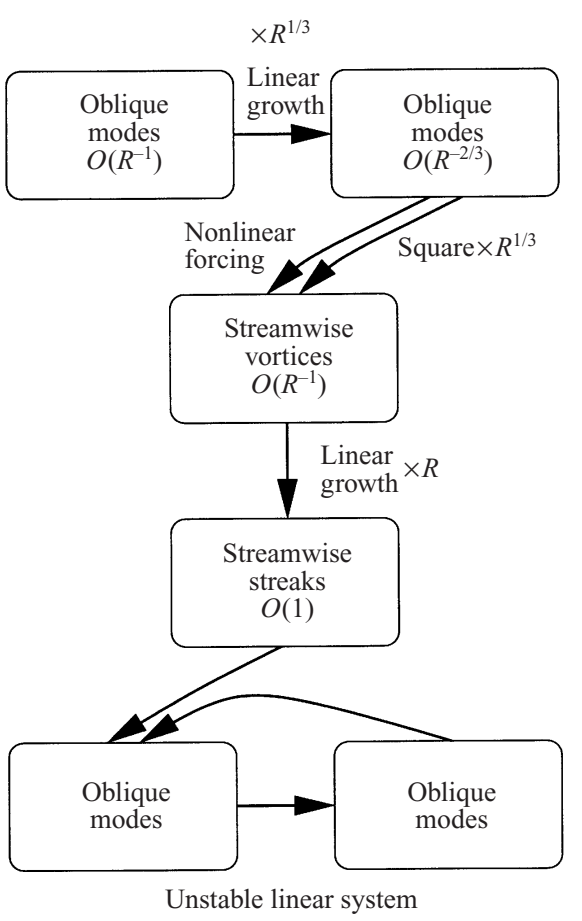

FIGURE 19. A schematic diagram showing the route to transition for plane Couette flow with the norm of the solution at each point.

In $\S 5$ we saw that the most sensitive eigenvalues are the centre modes. We found that a streamwise streak $\eta^{0 \beta_{1}}$ of order one was necessary to perturb the other modes at leading order, whereas an even $\eta^{0 \beta_{1}}$ of order $R^{-1 / 2}$, or an odd $\eta^{0 \beta_{1}}$ of order $R^{-1 / 4}$ is enough to perturb the centre modes at leading order. Hence for an odd initial perturbation $v_{0}^{0 \beta_{1}}$ the threshold amplitude for streamwise vortices is $R^{-3 / 2}$, while for an even initial perturbation $v_{0}^{0 \beta_{1}}$ the threshold amplitude is $R^{-5 / 4}$.

Since it is the centre modes which produce the secondary instability in plane Poiseuille flow the instability will be concentrated in the centre of the channel. Note also that in contrast to the Tollmien-Schlichting wave, which has $\alpha$ asymptotically small with $R$, the centre modes which become unstable are those with streamwise wavenumber $\alpha$ of order one.

Since the imaginary component of the perturbed eigenvalues is order $R^{-1 / 2}$, the growth rate of the instability will be order $R^{-1 / 2}$. For large values of $R$ this is much larger than the decay rate of the streamwise modes (which is order $R^{-1}$ ), so that once the secondary instability takes place the solution will proceed to enter the fully nonlinear regime of the equations and we expect to have transition from the laminar state. However, for Reynolds numbers in the range 1500 to 5000, the difference between these two timescales is not so great as it is in the case of plane Couette flow. Hence it may be possible to initiate a secondary instability which does not have time to grow enough to produce transition before the streamwise streak has decayed and stabilized the oblique modes. This may explain why the numerically determined threshold scalings for secondary instability and for full transition are the same for plane Couette flow, but differ for plane Poiseuille flow in the region $1500<R<5000$ (Reddy et al. 1998). 


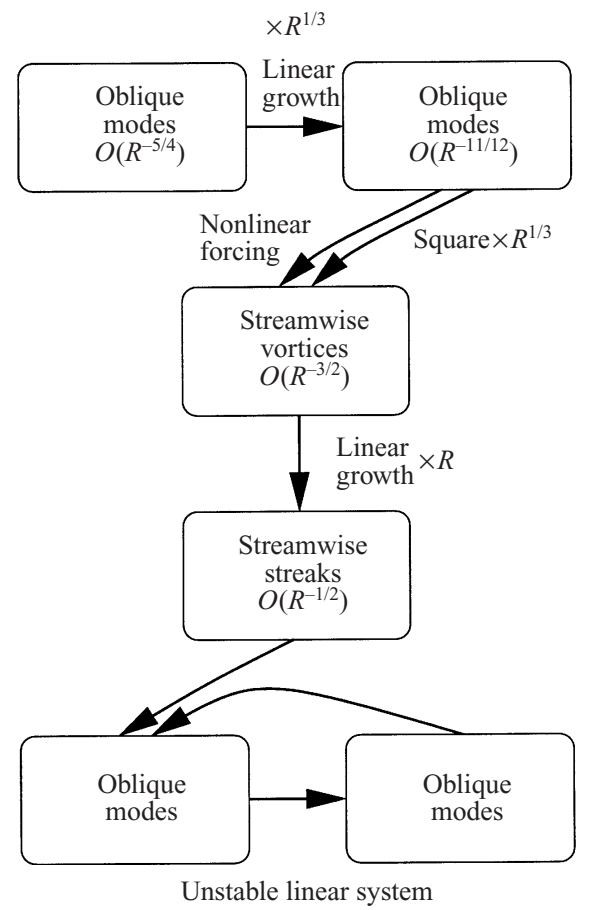

FIGURE 20. A schematic diagram showing the route to transition for plane Poiseuille flow with the norm of the solution at each point.

7.2.2. Route (ii): an initial perturbation in the form of optimally growing oblique modes

Now consider an initial condition in the form of optimally growing oblique modes, that is $\eta=0, v=v_{0}^{\alpha \beta}(y) \mathrm{e}^{\mathrm{i} \alpha x} \mathrm{e}^{\mathrm{i} \beta z}+v_{0}^{-\alpha \beta}(y) \mathrm{e}^{-\mathrm{i} \alpha x} \mathrm{e}^{\mathrm{i} \beta z}$ for some non-zero $\alpha$ and $\beta$, as for plane Couette flow.

The calculation of the size of oblique and streamwise modes generated by the nonlinear terms proceeds exactly as in the case of plane Couette flow. Thus an initial condition of order $v_{0}$ produces a streamwise vortex of order $R^{5 / 3} v_{0}^{2}$. Note also that the streamwise vortex produced is odd, whether the initial oblique perturbation is odd or even. Thus an oblique perturbation of order $R^{-19 / 12}$ will lead to transition. However, since $v_{0}$ oscillates rapidly in $y$ on the scale $R^{-1 / 3}$, the norm of this perturbation (both $L^{2}$ and $L^{\infty}$ ), which is the threshold amplitude, is of order $R^{-5 / 4}$.

In summary, the threshold amplitude for an initial perturbation in the form of a streamwise vortex is $O\left(R^{-5 / 4}\right)$ for even perturbations in $y$ and $O\left(R^{-3 / 2}\right)$ for odd perturbations in $y$, while the threshold amplitude for an initial perturbation in the form of oblique modes is $O\left(R^{-5 / 4}\right)$. In each case the final secondary instability occurs in the centre modes.

In figure 20 we show a schematic diagram of the route to transition showing the norm of the solution at each stage.

We have found that in the limit $R \rightarrow \infty$ a perturbation of $O\left(R^{-3 / 2}\right)$ is enough to produce a secondary instability in the centre modes, while leaving the neutral curve of the Tollmien-Schlichting mode unchanged. Thus our scenario for subcritical transition in plane Poiseuille flow is that for large but subcritical Reynolds numbers 
a small but finite perturbation will destabilize the centre modes while leaving the wall modes stable.

\subsection{Threshold amplitudes for transition in non-periodic flows}

The $L^{\infty}$ norm of the threshold amplitude of $\boldsymbol{u}$ scales-the same way for periodic and non-periodic flows; the separation between streamwise and oblique modes becomes asymptotic rather than sharp, but the analysis proceeds in exactly the same way. However, when the solution is not periodic we must be more careful in calculating its energy norm.

It is tempting just to let the period tend to infinity in (3.30), to give the RMS energy norm

$$
\lim _{l_{x}, l_{z} \rightarrow \infty}\left(\frac{1}{l_{x} l_{z}} \int_{0}^{l_{x}} \int_{0}^{l_{z}} \int_{-1}^{1}\left(u^{2}+v^{2}+w^{2}\right) \mathrm{d} y \mathrm{~d} z \mathrm{~d} x\right)^{1 / 2} .
$$

However, any perturbation localized over a finite region now has zero norm, and since such perturbations can clearly lead to transition the threshold amplitude is meaningless. On infinite domains we must instead consider the norm corresponding to the total energy of the perturbation

$$
\left(\frac{1}{2} \int_{-\infty}^{\infty} \int_{-\infty}^{\infty} \int_{-1}^{1}\left(u^{2}+v^{2}+w^{2}\right) \mathrm{d} y \mathrm{~d} z \mathrm{~d} x\right)^{1 / 2} .
$$

Now, though, a streamwise perturbation with $\alpha=0$ does not decay at infinity in $x$, and therefore has infinite norm. However, streamwise perturbations with $\alpha=O\left(R^{-1}\right)$ exhibit the same growth characteristics, and these extend a distance $O(R)$ in the streamwise direction, so that their norm is greater than the $L^{\infty}$ norm by a factor of order $R^{1 / 2}$. Likewise, for a pair of oblique modes to generate a streamwise vortex they must extend a distance $O(R)$ in the streamwise direction, so that here again the energy norm is greater than the $L^{\infty}$ norm by a factor of order $R^{1 / 2}$.

Because of these extra factors, the transition involving only oblique modes described in Appendix E may come into play, and a more careful analysis of it is required. In fact, when considering infinite domains it is probably better to work in the primary space of $x$ and $z$ rather than the Fourier space of $\alpha$ and $\beta$.

\section{Comparison with numerical experiments}

Numerical experiments by Lundbladh et al. (1994) seem to suggest that the threshold amplitude for plane Couette flow should scale like $R^{-1}$ for streamwise vortices and like $R^{-5 / 4}$ for oblique modes, while for plane Poiseuille flow it should scale like $R^{-7 / 4}$ for both streamwise vortices and oblique modes. Kreiss et al. (1994) also examine numerical evolution of a pair of streamwise vortices in Couette flow and find a threshold amplitude which scales like $R^{-1}$. Further experiments by Reddy et al. (1998) suggest that the amplitude of an initial streamwise vortex necessary to induce a secondary instability in the oblique modes scales like $R^{-1}$ and $R^{-1.6}$ for plane Couette flow and plane Poiseuille flow respectively. Our asymptotic results of $R^{-1}$ and $R^{-3 / 2}$ respectively agree reasonably well with these, and we already have an explanation in $\S 7.2 .1$ for the fact that, for not too large Reynolds numbers, there might be a difference between the amplitude necessary for transition and that necessary for secondary instability.

However, there is disagreement for the oblique-mode threshold in both plane Couette and plane Poiseuille flow, which we now try to explain. 


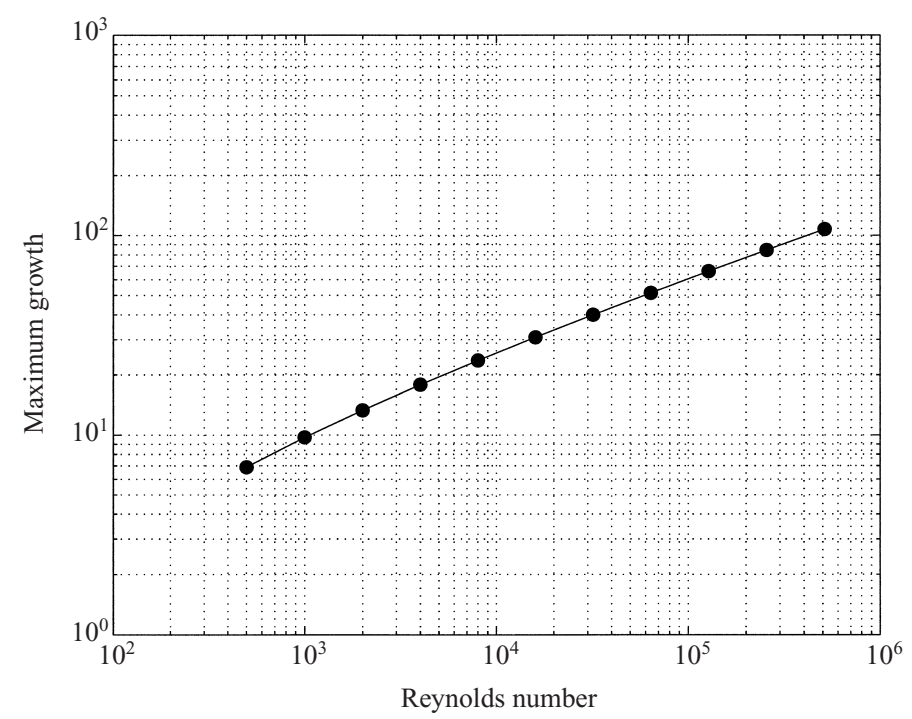

FIGURE 21. Numerically determined maximum growth for the plane Couette flow oblique mode $\alpha=1, \beta=1$. The linearized Navier-Stokes equations were solved in MATLAB using codes written by Satish Reddy based a Chebyshev hybrid spectral discretization, as described in Reddy \& Henningson (1993) and Reddy et al. (1993). The figure shows the maximum norm of $\exp (A(t))$, where the linearized system is written as $\boldsymbol{y}_{t}=A \boldsymbol{y}$.

\subsection{Plane Couette flow}

Figure 21 shows the numerically determined maximum growth of the norm of the solution of the linearized Navier-Stokes equations as a function of the Reynolds number for the oblique mode $\alpha=1, \beta=1$ in plane Couette flow. Figure 22 shows an approximation to the gradient of this curve, calculated using a simple central difference between successive points. We see that the curve takes a long time to approach its asymptote of $1 / 3$. The transient growth appears to scale like a much higher power of the Reynolds number than this for Reynolds numbers in the range $500 \leqslant R \leqslant 2000$, which is the range for the numerical experiments in Lundbladh et al. (1994).

Let us try and determine the effect of this on the threshold amplitude. Suppose that the transient growth in the oblique modes is $G$ and that it decays over a timescale $T$. Then if the initial norm of the perturbation is $N$, the nonlinear terms in (3.23) will produce a $v$ of size $T G^{2} N^{2}$. Hence, the condition for instability is $T G^{2} N^{2} \sim R^{-1}$, so that

$$
N \sim \frac{R^{-1 / 2}}{G T^{1 / 2}}
$$

Hence if the exponent of the growth in the oblique modes appears larger than it really is, the exponent of the threshold amplitude will appear smaller than the true asymptotic value. In figure 23 we show the result of using the numerically determined value of $G$ to estimate the scaling of $N$. Thus we have a possible explanation for why the threshold amplitude found in Lundbladh et al. (1994) for the range $500 \leqslant R \leqslant 2000$ is much lower than -1 .

\subsection{Plane Poiseuille flow}

Figure 24 shows the numerically determined maximum growth of the norm of the solution of the linearized equations as a function of the Reynolds number for the oblique mode $\alpha=1, \beta=1$ in plane Poiseuille flow (the maximum growth occurs for an even 


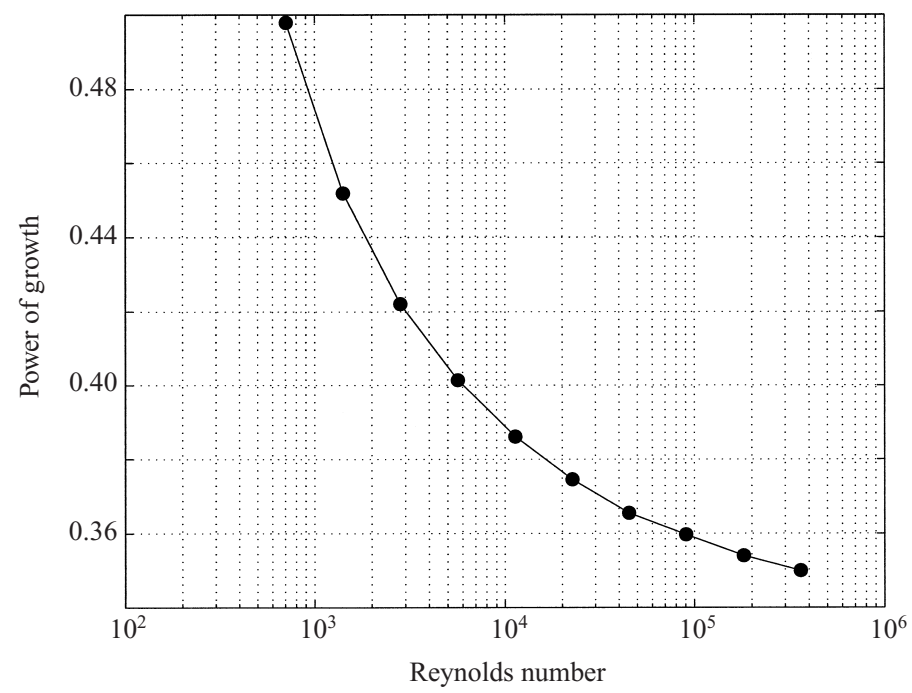

FIGURE 22. Numerically determined maximum growth exponent for the plane Couette flow oblique mode $\alpha=1, \beta=1$.

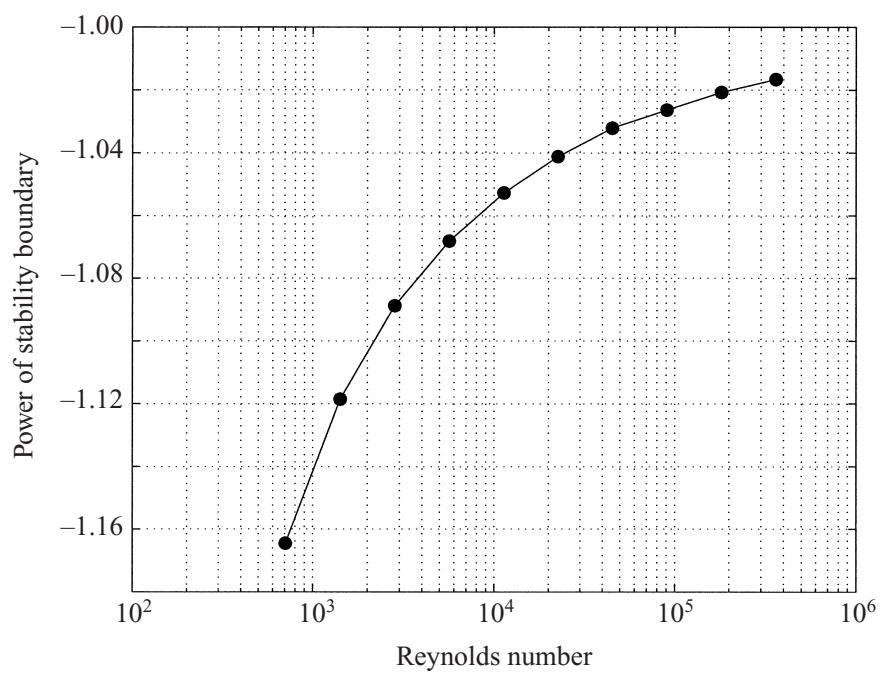

FIGURE 23. The power of the threshold amplitude for plane Couette flow based on the numerically determined transient growth for the oblique mode $\alpha=1, \beta=1$.

initial condition). Figure 25 shows the gradient of this curve, again computed from figure 24 using simple central differences. As before, the curve takes a long time to approach its asymptote of $1 / 3$. The transient growth appears to scale like a much higher power of the Reynolds number than this for Reynolds numbers in the range $1500 \leqslant$ $R \leqslant 5000$, which is the range for the numerical experiments in Lundbladh et al. (1994).

As before, suppose that the transient growth in the oblique modes is $G$ and that it decays over a timescale $T$. Then if the initial norm of the perturbation is $N$, the condition for instability is $T G^{2} N^{2} \sim R^{-3 / 2}$, so that

$$
N \sim \frac{R^{-3 / 4}}{G T^{1 / 2}} .
$$




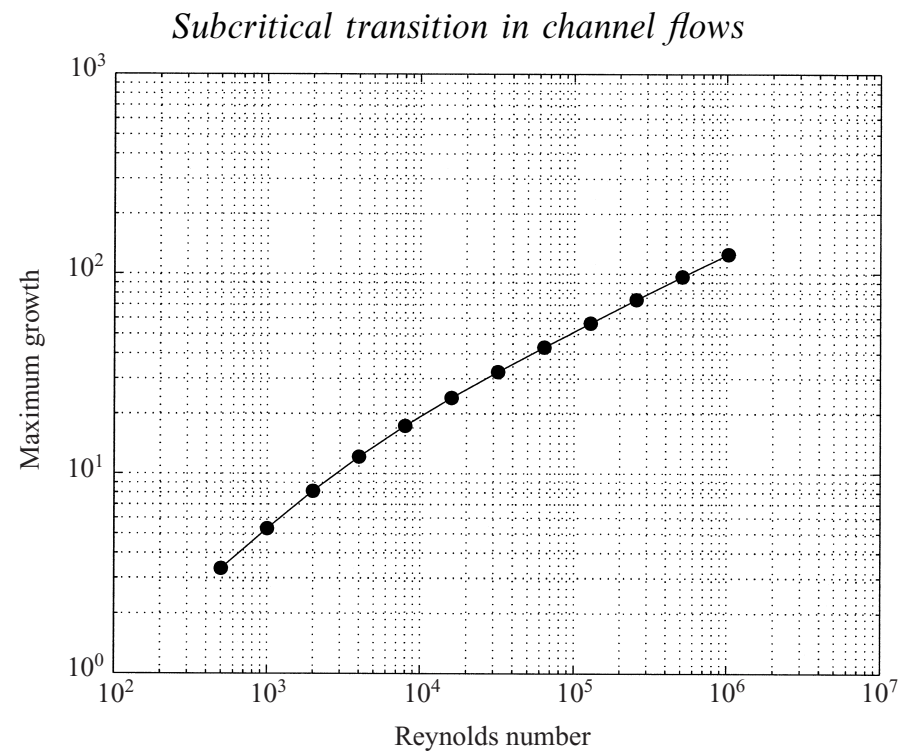

FiguRE 24. Numerically determined maximum growth for the plane Poiseuille flow oblique mode $\alpha=1, \beta=1$. The linearized Navier-Stokes equations were solved in MATLAB using codes written by Satish Reddy based a Chebyshev hybrid spectral discretization, as described in Reddy \& Henningson (1993) and Reddy et al. (1993). The figure shows the maximum norm of $\exp (A(t))$, where the linearized system is written as $\boldsymbol{y}_{t}=A \boldsymbol{y}$.

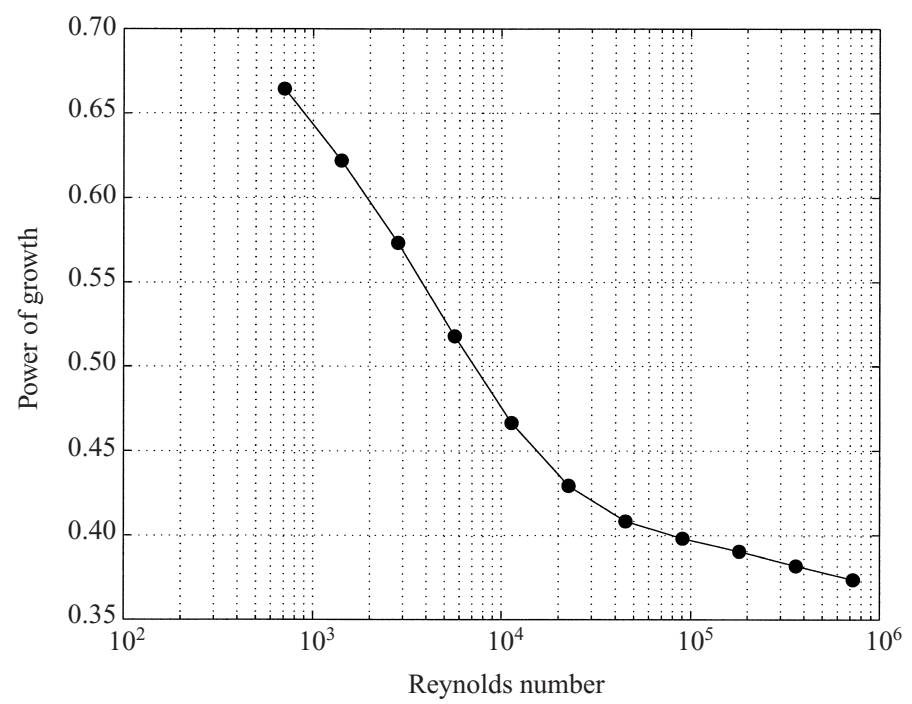

FIGURE 25. Numerically determined maximum growth exponent for the plane Poiseuille flow oblique mode $\alpha=1, \beta=1$.

The result of using the numerically determined value of $G$ to estimate $N$ is shown in figure 26

Thus we see again a possible reason why the threshold amplitude found in Lundbladh et al. (1994) for the range $1500 \leqslant R \leqslant 5000$ is much smaller that $-5 / 4$.

Finally we should indicate why the numerically determined exponent of the transient growth may converge so slowly to its asymptotic value. Suppose the growth rate is $G=A\left(R^{1 / 3}+B\right)$. In figures 22 and 25 we plot the gradient of $\log G$ with respect to 


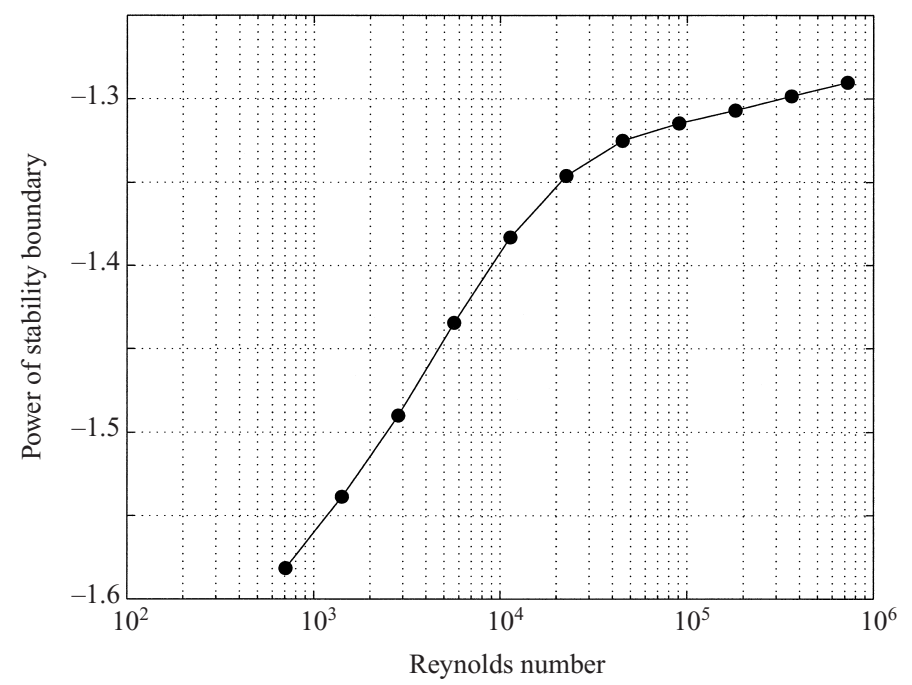

FIGURE 26. The power of the threshold amplitude for plane Poiseuille flow based on the numerically determined transient growth for the oblique mode $\alpha=1, \beta=1$.

$\begin{array}{lcc} & \text { Couette } & \text { Poiseuille } \\ \text { Streamwise } & -1 & -3 / 2 \\ \text { Oblique } & -1 & -5 / 4\end{array}$

TABLE 2. Asymptotically determined threshold exponents for initial perturbations in the form of optimally growing streamwise vortices and optimally growing oblique modes in plane Couette and plane Poiseuille flow.

$\log R$, which is

$$
\begin{aligned}
\frac{\mathrm{d} \log G}{\mathrm{~d} \log R} & =\frac{1}{3}+\frac{-B R^{-1 / 3}}{3\left(1+B R^{-1 / 3}\right)} \\
& \sim \frac{1}{3}-\frac{B R^{-1 / 3}}{3} .
\end{aligned}
$$

Now, at a Reynolds number of $1000, R^{-1 / 3}$ is only $1 / 10$, so that even $B=5$ will produce a $50 \%$ error in the numerically determined exponent.

\section{Conclusions}

We have examined the threshold amplitude for transition from the laminar state as the Reynolds number $R \rightarrow \infty$ through an analysis of the Navier-Stokes equations. We find that for plane Couette flow the threshold scales like $R^{-1}$ for the two routes to transition

(i) streamwise vortices $\rightarrow$ streamwise streaks

$\rightarrow$ secondary instability of oblique modes,

(ii) oblique modes $\rightarrow$ streamwise vortices $\rightarrow$ streamwise streaks

$\rightarrow$ secondary instability of oblique modes, 
while for plane Poiseuille flow the threshold scales like $R^{-5 / 4}$ and $R^{-3 / 2}$ for even and odd perturbations respectively via route (i), and like $R^{-5 / 4}$ via route (ii) (see table 2). We have also examined the boot-strapping route to transition

(iii) streamwise vortices $\rightarrow$ streamwise streaks $\rightarrow$ streamwise vortices

and have found that the threshold amplitude is of $O\left(R^{-1}\right)$ for both plane Couette and plane Poiseuille flow.

The secondary instability in the oblique modes in plane Couette flow occurs in all modes and will therefore be distributed throughout the channel, although the growth rate of the wall modes is much smaller than that of the others. The growth rate of the non-wall modes is of $O(1)$, while the streamwise streak generating the instability decays on a timescale of $O(R)$, so that the secondary instability has plenty of time to generate order-one oblique modes, entering the fully nonlinear regime in the equations, before the streak decays.

For plane Poiseuille flow the secondary instability is in the centre modes, which are localized near the centre of the channel. The instability is essentially an inviscid instability due to an inflectional profile in the crosswise coordinate $z$; the centre modes are the most sensitive since they are localized in a region of low shear. The growth rate of the instability is of $O\left(R^{-1 / 2}\right)$; in this case, even though there is a large difference in the timescales as $R \rightarrow \infty$, for finite $R$ they may not be so different and it is possible that the secondary instability may not have time to generate order-one oblique modes leading to full transition before the streamwise streak has decayed. This may explain the difference in the threshold amplitudes for secondary instability and transition observed numerically in Reddy et al. (1998). For the route to transition (i) we find that the threshold amplitude scales differently for odd and even perturbations, which agrees with the numerical findings of Lundbladh et al. (1994).

Our asymptotic results for threshold amplitudes for the route to transition (i) agree with the numerical results of Lundbladh et al. (1994). However, the results for the route to transition (ii) are not in such good agreement. This is because the transient growth in the oblique modes converges only slowly to its asymptotic value, which leads to an apparently lower threshold exponent at moderate Reynolds numbers.

For plane Couette flow and plane Poiseuille flow on a fixed periodic domain our results determine the scaling of the basin of attraction of the laminar state as $R \rightarrow \infty$. However, plane Poiseuille flow on an infinite domain is linearly unstable for large Reynolds numbers, so that our analysis needs more careful interpretation. We have determined the scaling of the domain of attraction of the stable modes of the system. While such an analysis is irrelevant for very large $R$ (since the unstable modes will dominate), we expect that subcritical transition for $R<5772$ is caused by the small domain of attraction of the laminar state, and that the origin of this and route to transition is qualitatively similar to that of the stable modes in the limit as $R \rightarrow \infty$.

We have examined explicitly the cases of plane Couette and plane Poiseuille flow, but the analysis is trivially extended to the case of a combined plane Poiseuille and plane Couette flow. The transient growth calculation is valid for arbitrary $U(y)$. The calculations of the perturbations of the eigenvalues follow as before. The only difference is that the centre modes exist if and only if there is a point of zero shear in the profile $U(y)$. If this is the case then the flow will basically behave like plane Poiseuille flow, while if not it will behave like plane Couette flow. Of course there is a special case when the zero shear point lies on the wall, but this is also easily treated.

While the asymptotic results compare resonably favourably with direct numerical simulations of the Navier-Stokes equations on periodic domains, it is very difficult to 
find suitable experimental data with which to compare. This is because the transient growth of streamwise vortices to streamwise streaks takes a long time (it occurs on the viscous timescale of order $R$ ): the streamwise vortex transfers momentum from the basic flow via the lift-up mechanism until it decays through the action of viscosity. Hence in an experimental situation the maximum transient growth will not be achieved until the perturbation has travelled a distance $O(R)$ (i.e. order Reynolds number channel half-widths) downstream. Experimental configurations often have very long pipes or channels, but this is to ensure that the boundary layers generated at the inlet have diffused to establish Poiseuille flow (which also takes a distance $O(R)$ ). A perturbation is induced and typically transition is looked for a few pipe diameters or channel widths downstream. An optimal perturbation of the type described here would be out of the end of the pipe or channel before it had had a chance to induce transition.

There are two conclusions we can draw from this: (i) if the threshold amplitude is to be measured experimentally it is necessary to have a pipe which is $O(R)$ radii long both upstream and downstream of the induced perturbation, and (ii) the threshold amplitude described in this paper, although optimal for infinite channels, may be increased for finite channels. With a finite channel it is necessary to place restrictions on the time taken to achieve transition. The transient growth of streamwise modes will be reduced in proportion to the time allowed, which will correspondingly raise the threshold amplitude.

The author would like to thank Professor L. N. Trefethen for introducing him to the subject of subcritical transition, for many useful discussions, and for many helpful suggestions on the presentation of this work, including in particular the suggestion to include tables 1 and 2 and figures $1-3$ and 19-20. The numerical codes used to solve the linearized Navier-Stokes equations were written in MATLAB by Professor S. C. Reddy, based on Fortran codes written by Professor D. S. Henningson. The author would like to thank Professor D. S. Henningson, Dr S. J. Cowley, Professor P. Duck and Prof P. G. Drazin for guiding him towards relevant literature. The author also gratefully acknowledges many useful discussions with Dr J. R. Ockendon. Finally, the author would like to thank the referees for their many helpful comments.

\section{Appendix A. Nonlinear terms in the Navier-Stokes equations}

The nonlinear terms in (3.23) are given explicitly by

$$
\begin{aligned}
& N_{1}^{\alpha_{1} \beta_{1} \alpha_{2} \beta_{2}}\left(v^{\alpha_{1} \beta_{1}}, \eta^{\alpha_{1} \beta_{1}}, v^{\alpha_{2} \beta_{2}}, \eta^{\alpha_{2} \beta_{2}}\right)=\frac{\alpha_{1} \alpha_{2}+\beta_{1} \beta_{2}+k_{2}^{2}}{k_{2}^{2}} v^{\alpha_{1} \beta_{1}} v_{y y y}^{\alpha_{2} \beta_{2}} \\
& +\frac{\alpha_{1} \alpha_{2}+\beta_{1} \beta_{2}+k_{1}^{2}}{k_{1}^{2}} v^{\alpha_{2} \beta_{2}} v_{y y y}^{\alpha_{1} \beta_{1}} \\
& +\frac{-\alpha_{1}^{2} \alpha_{2}^{2}-\alpha_{1} \alpha_{2}^{3}+\alpha_{2}^{2} \beta_{1}^{2}-4 \alpha_{1} \alpha_{2} \beta_{1} \beta_{2}-\alpha_{2}^{2} \beta_{1} \beta_{2}+\alpha_{1}^{2} \beta_{2}^{2}-\alpha_{1} \alpha_{2} \beta_{2}^{2}-\beta_{1}^{2} \beta_{2}^{2}-\beta_{1} \beta_{2}^{3}}{k_{1}^{2} k_{2}^{2}} \\
& \quad \times v_{y}^{\alpha_{1} \beta_{1}} v_{y y}^{\alpha_{2} \beta_{2}} \\
& +\frac{-\alpha_{1}^{2} \alpha_{2}^{2}-\alpha_{1}^{3} \alpha_{2}+\alpha_{2}^{2} \beta_{1}^{2}-4 \alpha_{1} \alpha_{2} \beta_{1} \beta_{2}-\alpha_{1}^{2} \beta_{1} \beta_{2}+\alpha_{1}^{2} \beta_{2}^{2}-\alpha_{1} \alpha_{2} \beta_{1}^{2}-\beta_{1}^{2} \beta_{2}^{2}-\beta_{1}^{3} \beta_{2}}{k_{1}^{2} k_{2}^{2}} \\
& \quad \times v_{y y}^{\alpha_{1} \beta_{1}} v_{y}^{\alpha_{2} \beta_{2}}
\end{aligned}
$$




$$
\begin{aligned}
& +\frac{2 \alpha_{1} \alpha_{2}^{2} \beta_{1}+\alpha_{2}^{3} \beta_{1}-2 \alpha_{1}^{2} \alpha_{2} \beta_{2}-\alpha_{1} \alpha_{2}^{2} \beta_{2}+2 \alpha_{2} \beta_{1}^{2} \beta_{2}-2 \alpha_{1} \beta_{1} \beta_{2}^{2}+\alpha_{2} \beta_{1} \beta_{2}^{2}-\alpha_{1} \beta_{2}^{3}}{k_{1}^{2} k_{2}^{2}} \\
& \times \eta^{\alpha_{1} \beta_{1}} v_{y y}^{\alpha_{2} \beta_{2}} \\
& +\frac{2 \alpha_{2} \alpha_{1}^{2} \beta_{2}+\alpha_{1}^{3} \beta_{2}-2 \alpha_{2}^{2} \alpha_{1} \beta_{1}-\alpha_{2} \alpha_{1}^{2} \beta_{1}+2 \alpha_{1} \beta_{2}^{2} \beta_{1}-2 \alpha_{2} \beta_{2} \beta_{1}^{2}+\alpha_{1} \beta_{2} \beta_{1}^{2}-\alpha_{2} \beta_{1}^{3}}{k_{1}^{2} k_{2}^{2}} \\
& \times \eta^{\alpha_{2} \beta_{2}} v_{y y}^{\alpha_{1} \beta_{1}} \\
& +\frac{\alpha_{2} \beta_{1}-\alpha_{1} \beta_{2}}{k_{2}^{2}} v^{\alpha_{1} \beta_{1}} \eta_{y y}^{\alpha_{2} \beta_{2}}+\frac{\alpha_{1} \beta_{2}-\alpha_{2} \beta_{1}}{k_{1}^{2}} v^{\alpha_{2} \beta_{2}} \eta_{y y}^{\alpha_{1} \beta_{1}} \\
& +\frac{2\left(\alpha_{1} \alpha_{2}^{2} \beta_{1}-\alpha_{1}^{2} \alpha_{2} \beta_{2}+\alpha_{2} \beta_{1}^{2} \beta_{2}-\alpha_{1} \beta_{1} \beta_{2}^{2}\right)}{k_{1}^{2} k_{2}^{2}} \eta_{y}^{\alpha_{1} \beta_{1}} v_{y}^{\alpha_{2} \beta_{2}} \\
& +\frac{2\left(\alpha_{2} \alpha_{1}^{2} \beta_{2}-\alpha_{2}^{2} \alpha_{1} \beta_{1}+\alpha_{1} \beta_{2}^{2} \beta_{1}-\alpha_{2} \beta_{2} \beta_{1}^{2}\right)}{k_{1}^{2} k_{2}^{2}} \eta_{y}^{\alpha_{2} \beta_{2}} v_{y}^{\alpha_{1} \beta_{1}} \\
& +\frac{\alpha_{1}^{3} \alpha_{2}+\alpha_{1}^{2} \alpha_{2}^{2}-\alpha_{1} \alpha_{2}^{3}-\alpha_{2}^{4}+\alpha_{1} \alpha_{2} \beta_{1}^{2}-\alpha_{2}^{2} \beta_{1}^{2}+\alpha_{1}^{2} \beta_{1} \beta_{2}+4 \alpha_{1} \alpha_{2} \beta_{1} \beta_{2}}{k_{2}^{2}} v^{\alpha_{1} \beta_{1}} v_{y}^{\alpha_{2} \beta_{2}} \\
& +\frac{-\alpha_{2}^{2} \beta_{1} \beta_{2}+\beta_{1}^{3} \beta_{2}-\alpha_{1}^{2} \beta_{2}^{2}-\alpha_{1} \alpha_{2} \beta_{2}^{2}-2 \alpha_{2}^{2} \beta_{2}^{2}+\beta_{1}^{2} \beta_{2}^{2}-\beta_{1} \beta_{2}^{3}-\beta_{2}^{4}}{k_{2}^{2}} v^{\alpha_{1} \beta_{1}} v_{y}^{\alpha_{2} \beta_{2}} \\
& +\frac{\alpha_{2}^{3} \alpha_{1}+\alpha_{2}^{2} \alpha_{1}^{2}-\alpha_{2} \alpha_{1}^{3}-\alpha_{1}^{4}+\alpha_{2} \alpha_{1} \beta_{2}^{2}-\alpha_{1}^{2} \beta_{2}^{2}+\alpha_{2}^{2} \beta_{2} \beta_{1}+4 \alpha_{2} \alpha_{1} \beta_{2} \beta_{1}}{k_{1}^{2}} v^{\alpha_{2} \beta_{2}} v_{y}^{\alpha_{1} \beta_{1}} \\
& +\frac{-\alpha_{1}^{2} \beta_{2} \beta_{1}+\beta_{2}^{3} \beta_{1}-\alpha_{2}^{2} \beta_{1}^{2}-\alpha_{2} \alpha_{1} \beta_{1}^{2}-2 \alpha_{1}^{2} \beta_{1}^{2}+\beta_{2}^{2} \beta_{1}^{2}-\beta_{2} \beta_{1}^{3}-\beta_{1}^{4}}{k_{1}^{2}} v^{\alpha_{2} \beta_{2}} v_{y}^{\alpha_{1} \beta_{1}} \\
& +\frac{2\left(\alpha_{2} \beta_{1}-\alpha_{1} \beta_{2}\right)^{2}}{k_{1}^{2} k_{2}^{2}}\left(\eta^{\alpha_{1} \beta_{1}} \eta_{y}^{\alpha_{2} \beta_{2}}+\eta^{\alpha_{2} \beta_{2}} \eta_{y}^{\alpha_{1} \beta_{1}}\right) \\
& +\frac{-\alpha_{1}^{2} \alpha_{2} \beta_{1}-2 \alpha_{1} \alpha_{2}^{2} \beta_{1}-\alpha_{2}^{3} \beta_{1}-\alpha_{2} \beta_{1}^{3}+\alpha_{1}^{3} \beta_{2}+2 \alpha_{1}^{2} \alpha_{2} \beta_{2}+\alpha_{1} \alpha_{2}^{2} \beta_{2}}{k_{1}^{2}} \eta^{\alpha_{1} \beta_{1}} v^{\alpha_{2} \beta_{2}} \\
& +\frac{\alpha_{1} \beta_{1}^{2} \beta_{2}-2 \alpha_{2} \beta_{1}^{2} \beta_{2}+2 \alpha_{1} \beta_{1} \beta_{2}^{2}-\alpha_{2} \alpha_{1} \beta_{2}^{2}+\alpha_{1} \beta_{2}^{3}}{k_{1}^{2}} \eta^{\alpha_{1} \beta_{1}} v^{\alpha_{2} \beta_{2}} \\
& +\frac{-\alpha_{2}^{2} \alpha_{1} \beta_{2}-2 \alpha_{2} \alpha_{1}^{2} \beta_{2}-\alpha_{1}^{3} \beta_{2}-\alpha_{1} \beta_{2}^{3}+\alpha_{2}^{3} \beta_{1}+2 \alpha_{2}^{2} \alpha_{1} \beta_{1}+\alpha_{2} \alpha_{1}^{2} \beta_{1}}{k_{2}^{2}} \eta^{\alpha_{2} \beta_{2}} v^{\alpha_{1} \beta_{1}} \\
& +\frac{\alpha_{2} \beta_{2}^{2} \beta_{1}-2 \alpha_{1} \beta_{2}^{2} \beta_{1}+2 \alpha_{2} \beta_{2} \beta_{1}^{2}-\alpha_{1} \alpha_{2} \beta_{1}^{2}+\alpha_{2} \beta_{1}^{3}}{k_{2}^{2}} \eta^{\alpha_{2} \beta_{2}} v^{\alpha_{1} \beta_{1}},
\end{aligned}
$$

where $k_{1}^{2}=\alpha_{1}^{2}+\beta_{1}^{2}, k_{2}^{2}=\alpha_{2}^{2}+\beta_{2}^{2}$. The nonlinear terms in (3.24) are given by

$$
\begin{aligned}
& N_{2}^{\alpha_{1} \beta_{1} \alpha_{2} \beta_{2}}\left(v^{\alpha_{1} \beta_{1}}, \eta^{\alpha_{1} \beta_{1}}, v^{\alpha_{2} \beta_{2}}, \eta^{\alpha_{2} \beta_{2}}\right)=\frac{\left(\alpha_{1} \beta_{2}-\alpha_{2} \beta_{1}\right)\left(k_{2}^{2}-k_{1}^{2}\right)}{k_{1}^{2} k_{2}^{2}} \eta^{\alpha_{1} \beta_{1}} \eta^{\alpha_{2} \beta_{2}} \\
& -\frac{\alpha_{1} \beta_{2}-\alpha_{2} \beta_{1}}{k_{2}^{2}} v^{\alpha_{1} \beta_{1}} v_{y y}^{\alpha_{2} \beta_{2}}+\frac{\alpha_{1} \beta_{2}-\alpha_{2} \beta_{1}}{k_{1}^{2}} v^{\alpha_{2} \beta_{2}} v_{y y}^{\alpha_{1} \beta_{1}} \\
& -\frac{\alpha_{1} \alpha_{2}+\beta_{1} \beta_{2}+k_{2}^{2}}{k_{2}^{2}}\left(v^{\alpha_{1} \beta_{1}} \eta_{y}^{\alpha_{2} \beta_{2}}-\eta^{\alpha_{1} \beta_{1}} v_{y}^{\alpha_{2} \beta_{2}}\right) \\
& -\frac{\alpha_{1} \alpha_{2}+\beta_{1} \beta_{2}+k_{1}^{2}}{k_{1}^{2}}\left(v^{\alpha_{2} \beta_{2}} \eta_{y}^{\alpha_{1} \beta_{1}}-\eta^{\alpha_{2} \beta_{2}} v_{y}^{\alpha_{1} \beta_{1}}\right) .
\end{aligned}
$$




\section{Appendix B. Poor numerical agreement with the asymptotics for even modes}

The reason for the poor agreement between the asymptotic and numerical results in figure 18 is that when $\hat{A}$ and $\hat{B}$ are chosen to satisfy the leading-order behaviour in (5.28), namely

$$
\hat{A} v_{1}+\left(\hat{B}-b(\alpha R)^{3 / 4}\right) v_{2} \sim 0
$$

then

$$
\hat{A} v_{1}^{\prime}+\left(\hat{B}-b(\alpha R)^{3 / 4}\right) v_{2}^{\prime}
$$

is close to zero, so that the eigenvalues are pushed closer to the anti-Stokes line. To see why this is so we examine the inviscid solutions as $k^{2}(\lambda-1) \rightarrow 0$. The inviscid solutions satisfy

$$
\left(\lambda-1+y^{2}\right)\left(v_{y y}-k^{2} v\right)-2 v=0 .
$$

Let $y=\mathrm{i}(\lambda-1)^{1 / 2}$ s. Then

$$
\left(1-s^{2}\right)\left(v_{s s}+k^{2}(\lambda-1) v\right)+2 v=0 .
$$

We have the asymptotic behaviour

$$
\begin{aligned}
& v_{1} \sim 1+(s-1) \log (s-1), \\
& v_{2} \sim \mathrm{i}(\lambda-1)^{1 / 2}(s-1),
\end{aligned}
$$

as $s \rightarrow 1$. If we expand in powers of $k^{2}(\lambda-1)$ we find that

$$
\begin{aligned}
v_{1} & =s+\left(1-s^{2}\right) \tanh ^{-1} s+\cdots \\
& =\frac{1}{\mathrm{i}(\lambda-1)^{1 / 2}}\left(y+\frac{y^{2}+\lambda-1}{(\lambda-1)^{1 / 2}} \tan ^{-1}\left(\frac{y}{(\lambda-1)^{1 / 2}}\right)\right)+\cdots, \\
v_{2} & =-\frac{\mathrm{i}(\lambda-1)^{1 / 2}}{2}\left(1-s^{2}\right)+\cdots=\frac{y^{2}+\lambda-1}{2 i(\lambda-1)^{1 / 2}}+\cdots .
\end{aligned}
$$

The key point is that $v_{2}$ is even. This means that for the even wall modes, to satisfy the conditions at $y=0$ we must choose $\hat{A}=0$. Then to satisfy the conditions at $y=-1$ we must have $\hat{B} \approx b(\alpha R)^{3 / 4}$. But then

$$
\hat{A} v_{1}^{\prime}+\left(\hat{B}-b(\alpha R)^{3 / 4}\right) v_{2}^{\prime} \approx 0,
$$

so that $\exp (\operatorname{Re}( \pm \phi))=o\left(R^{1 / 4}\right)$. Thus for the even modes the eigenvalues move closer to the Squire modes. Note that this does not happen with the odd modes.

It is possible to perform a double expansion in $k^{2}(\lambda-1)$ and $R$ to obtain a closer fit with the numerical results, but we do not pursue such a line further here, since we are interested only in the asymptotic position of the eigenvalues as $R \rightarrow \infty$.

\section{Appendix C. Closeness of the eigenvalues for even and odd modes}

It is interesting that the even and odd eigenvalues on the right-hand branch of the ' $Y$ ' for plane Poiseuille flow are very nearly equal. Ng \& Reid (1999) have recently examined the outer limit of the inner problem (5.44) in the limit $\alpha \rightarrow 0$ to throw light on this curiosity. Here we show that the reason for this can again be explained by considering the inviscid solutions as $\lambda \rightarrow 1$, i.e. we consider the inner limit of the outer problem (without the restriction of small $\alpha$ ). 


\section{C.1. Even modes}

Since in this limit $v_{1}$ is odd, $v_{2}$ is even, (5.26)-(5.27) become

$$
\begin{gathered}
\hat{A} v_{1}^{\prime}+(\alpha R)^{1 / 2}\left(\phi^{\prime}\right)^{-3 / 2} \mathrm{e}^{(\alpha R)^{1 / 2} \phi}-\left(a+\hat{A} d(\alpha R)^{-3 / 4}\right)(\alpha R)^{1 / 2}\left(\phi^{\prime}\right)^{-3 / 2} \mathrm{e}^{-(\alpha R)^{1 / 2} \phi} \sim 0, \\
\hat{A} v_{1}^{\prime \prime \prime}+\left(\phi^{\prime}\right)^{1 / 2}(\alpha R)^{3 / 2} \mathrm{e}^{(\alpha R)^{1 / 2} \phi}-\left(a+\hat{A} d(\alpha R)^{-3 / 4}\right)\left(\phi^{\prime}\right)^{1 / 2}(\alpha R)^{3 / 2} \mathrm{e}^{-(\alpha R)^{1 / 2} \phi} \sim 0 .
\end{gathered}
$$

Thus $\hat{A} \approx 0$ (and hence $\hat{B} \approx 0$ by the boundary conditions at $y=-1$ ). Hence the eigenvalues lie approximately at the zeros of

$$
\mathrm{e}^{(\alpha R)^{1 / 2} \phi}-a \mathrm{e}^{-(\alpha R)^{1 / 2} \phi} .
$$

These are interspersed with the Squire modes, which lie approximately at the zeros of

$$
\mathrm{e}^{(\alpha R)^{1 / 2} \phi}+a \mathrm{e}^{-(\alpha R)^{1 / 2} \phi} \text {. }
$$

\section{C.2. Odd modes}

With $v_{1}$ odd and $v_{2}$ even (5.24)-(5.25) become

$$
\begin{gathered}
\left(\hat{B}+b(\alpha R)^{3 / 4}\right) v_{2}+\left(\phi^{\prime}\right)^{-5 / 2} \mathrm{e}^{(\alpha R)^{1 / 2} \phi}+\left(a+\hat{A} d(\alpha R)^{-3 / 4}\right) \mathrm{e}^{-(\alpha R)^{1 / 2} \phi} \sim 0, \\
\left(\hat{B}+b(\alpha R)^{3 / 4}\right) v_{2}^{\prime \prime}+\left(\phi^{\prime}\right)^{-1 / 2} \alpha R \mathrm{e}^{(\alpha R)^{1 / 2} \phi} \\
+\left(a+\hat{A} d(\alpha R)^{-3 / 4}\right)\left(\phi^{\prime}\right)^{-1 / 2} \alpha R \mathrm{e}^{-(\alpha R)^{1 / 2} \phi} \sim 0 .
\end{gathered}
$$

Hence $\hat{B} \approx-b(\alpha R)^{3 / 4}$. Now, by the boundary condition at $y=-1$,

$$
\hat{A} v_{1}(-1)+\hat{B} v_{2}(-1) \sim 0,
$$

so that

$$
\hat{A} \sim-\frac{\hat{B} v_{2}(-1)}{v_{1}(-1)} .
$$

Hence the eigenvalues for the odd modes lie approximately at the zeros of

$$
\mathrm{e}^{R^{1 / 2} \phi}+\left(a+\frac{b d v_{2}(-1)}{v_{1}(-1)}\right) \mathrm{e}^{-R^{1 / 2} \phi} .
$$

The reason that these are almost equal to the eigenvalues of the even modes is that, remarkably,

At $y=-1$ we find

$$
\frac{b d v_{2}(-1)}{v_{1}(-1)} \sim-2 a \text {. }
$$

$$
\begin{aligned}
& v_{1}(-1) \sim \frac{1}{\mathrm{i}(\lambda-1)^{1 / 2}}\left(-1+\frac{\lambda}{(\lambda-1)^{1 / 2}} \tan ^{-1}\left(\frac{-1}{(\lambda-1)^{1 / 2}}\right)\right), \\
& v_{2}(-1) \sim \frac{\lambda}{2 \mathrm{i}(\lambda-1)^{1 / 2}},
\end{aligned}
$$

giving

$$
\frac{v_{1}(-1)}{v_{2}(-1)}=\frac{2}{\lambda}\left(-1+\frac{\lambda}{(\lambda-1)^{1 / 2}} \tan ^{-1}\left(\frac{-1}{(\lambda-1)^{1 / 2}}\right)\right) \sim-\frac{\pi}{(\lambda-1)^{1 / 2}}
$$


as $\lambda \rightarrow 1$. Now, in Appendix D we show that

$$
\begin{aligned}
& a=-\mathrm{i}, \\
& b \sim \frac{2^{1 / 2} \pi^{1 / 2} \mathrm{e}^{3 i \pi / 8}}{(\lambda-1)^{1 / 4}}, \\
& d \sim \frac{2^{1 / 2} \pi^{1 / 2} \mathrm{e}^{\mathrm{i} \pi / 8}}{(\lambda-1)^{1 / 4}} .
\end{aligned}
$$

in the limit $\lambda \rightarrow 1$. Hence

$$
\frac{b d v_{2}(-1)}{v_{1}(-1)} \sim 2 \mathrm{i}=-2 a .
$$

\section{Appendix D. Stokes multipliers}

\section{D.1. Stokes multipliers (i) inviscid to $\mathrm{WK}$}

Consider first the Stokes multiplier $c$. We can determine it by a local examination in the vicinity of $\mathrm{i}(\lambda-1)^{1 / 2}$. Let

$$
\begin{aligned}
& v=1-\frac{\mathrm{i}}{(\lambda-1)^{1 / 2}}\left(y-\mathrm{i}(\lambda-1)^{1 / 2}\right) \log \left(y-\mathrm{i}(\lambda-1)^{1 / 2}\right)-\frac{\mathrm{i}(2 \alpha R)^{-1 / 3}}{(\lambda-1)^{2 / 3}} \bar{v}, \\
& y=\mathrm{i}(\lambda-1)^{1 / 2}+(2 \alpha R)^{-1 / 3}(\lambda-1)^{-1 / 6} s .
\end{aligned}
$$

Then

with

$$
\bar{v}_{s s s}-s \bar{v}_{s s}=-\frac{2}{s^{3}},
$$

With a series expansion

$$
\bar{v} \sim-\frac{1}{3 s^{2}} \quad \text { as } \quad s \rightarrow \infty .
$$

$$
v \sim \sum_{n=1}^{\infty} \frac{c_{n}}{s^{2+3 n}}
$$

we find

$$
c_{n}=\frac{c_{n-1}(3 n-1)(3 n)(3 n+1)}{3(n+1)},
$$

so that

$$
c_{n}=-\frac{(3 n+1) !}{3^{n+1}(n+1) !} .
$$

Thus

$$
\bar{v} \sim-\sum_{n=1}^{\infty} \frac{(3 n+1) !}{3^{n+1}(n+1) ! s^{2+3 n}} .
$$

Now,

$$
\frac{(3 n+1) !}{3^{n+1}(n+1) !} \sim \frac{3^{1 / 2}}{(2 \pi)^{1 / 2}}\left(\frac{3}{2}\right)^{2 n} \Gamma(2 n+1 / 2)
$$

as $n \rightarrow \infty$. Hence, as $n \rightarrow \infty$,

$$
\bar{v}_{n} \sim-\frac{\Gamma(2 n+1 / 2)}{\pi^{1 / 2} s^{5 / 4}}\left(\frac{3}{2 s^{3 / 2}}\right)^{2 n+1 / 2} .
$$


The methods of Daalhuis et al. (1995) and Chapman, King \& Adams (1998) which use matched asymptotic expansions to 'smooth' the Stokes discontinuity can then be employed to determine the Stokes multiplier. The Stokes line is at $\arg (s)=-2 \pi / 3$, across which the WKB solution turned on in $\bar{v}$ is

$$
-\frac{\pi^{1 / 2}}{s^{5 / 4}} \exp \left(2 s^{3 / 2} / 3\right)
$$

which is

$$
-\frac{\mathrm{i}(2 \alpha R)^{-1 / 3}}{(c-1)^{2 / 3}} \frac{\pi^{1 / 2}}{s^{5 / 4}} \exp \left(2 s^{3 / 2} / 3\right),
$$

in $v$. Now, in the inner coordinates,

$$
v_{4} \sim \frac{(\alpha R)^{5 / 12}}{2^{5 / 6} \mathrm{e}^{5 i \pi / 8}(\lambda-1)^{5 / 12} s^{5 / 4}} \exp \left(2 s^{3 / 2} / 3\right) .
$$

Hence the Stokes multiplier is

$$
\frac{2^{1 / 2} \pi^{1 / 2} \mathrm{e}^{\mathrm{i} \pi / 8}}{(\lambda-1)^{1 / 4}(\alpha R)^{3 / 4}}
$$

Thus

$$
d=\frac{2^{1 / 2} \pi^{1 / 2} \mathrm{e}^{\mathrm{i} \pi / 8}}{(\lambda-1)^{1 / 4}} .
$$

\section{D.2. Stokes multipliers (ii) WKB to inviscid}

Now let us consider the Stokes multiplier $b$, that is we consider the solution $v_{3}$ in the vicinity of $\mathrm{i}(\lambda-1)^{1 / 2}$. Since in the inner coordinates

$$
v_{3} \sim \frac{(\alpha R)^{5 / 12}}{2^{5 / 6} \mathrm{e}^{5 i \pi / 8}(\lambda-1)^{5 / 12} s^{5 / 4}} \exp \left(-2 s^{3 / 2} / 3\right),
$$

let

$$
\begin{gathered}
v=\frac{(\alpha R)^{5 / 12}}{2^{5 / 6} \mathrm{e}^{5 \mathrm{i} \pi / 8}(\lambda-1)^{5 / 12}} \bar{v} \\
y=\mathrm{i}(\lambda-1)^{1 / 2}+(2 \alpha R)^{-1 / 3}(\lambda-1)^{-1 / 6} \mathrm{~s} .
\end{gathered}
$$

Then

$$
\bar{v}_{s s s s}-s \bar{v}_{s s}=0,
$$

with

$$
\bar{v} \sim \frac{\exp \left(-2 s^{3 / 2} / 3\right)}{s^{5 / 4}} \text { as } s \rightarrow \infty .
$$

In this case, rather than using the series and smoothing the Stokes line, it is easier to write the solution to (D 4) as

$$
\bar{v}=2 \pi^{1 / 2} \int_{s}^{\infty} \int_{p}^{\infty} \operatorname{Ai}(q) \mathrm{d} q \mathrm{~d} p=2 \pi^{1 / 2} \int_{s}^{\infty} \int_{s}^{q} \operatorname{Ai}(q) \mathrm{d} p \mathrm{~d} q=2 \pi^{1 / 2} \int_{s}^{\infty}(q-s) \operatorname{Ai}(q) \mathrm{d} q .
$$

For $-2 \pi / 3<\arg (s)<2 \pi / 3$ as $s \rightarrow \infty$ The steepest descent path is as shown in figure $27(a)$, and the only contribution is from the endpoint, which gives

$$
\bar{v} \sim 2 \pi^{1 / 2} \int_{0}^{\infty} \frac{\exp \left(-2 s^{3 / 2} / 3\right)}{2 \pi^{1 / 2} s^{1 / 4}} \bar{q} \exp \left(-s^{1 / 2} \bar{q}\right) \mathrm{d} q=\frac{\exp \left(-2 s^{3 / 2} / 3\right)}{s^{5 / 4}},
$$



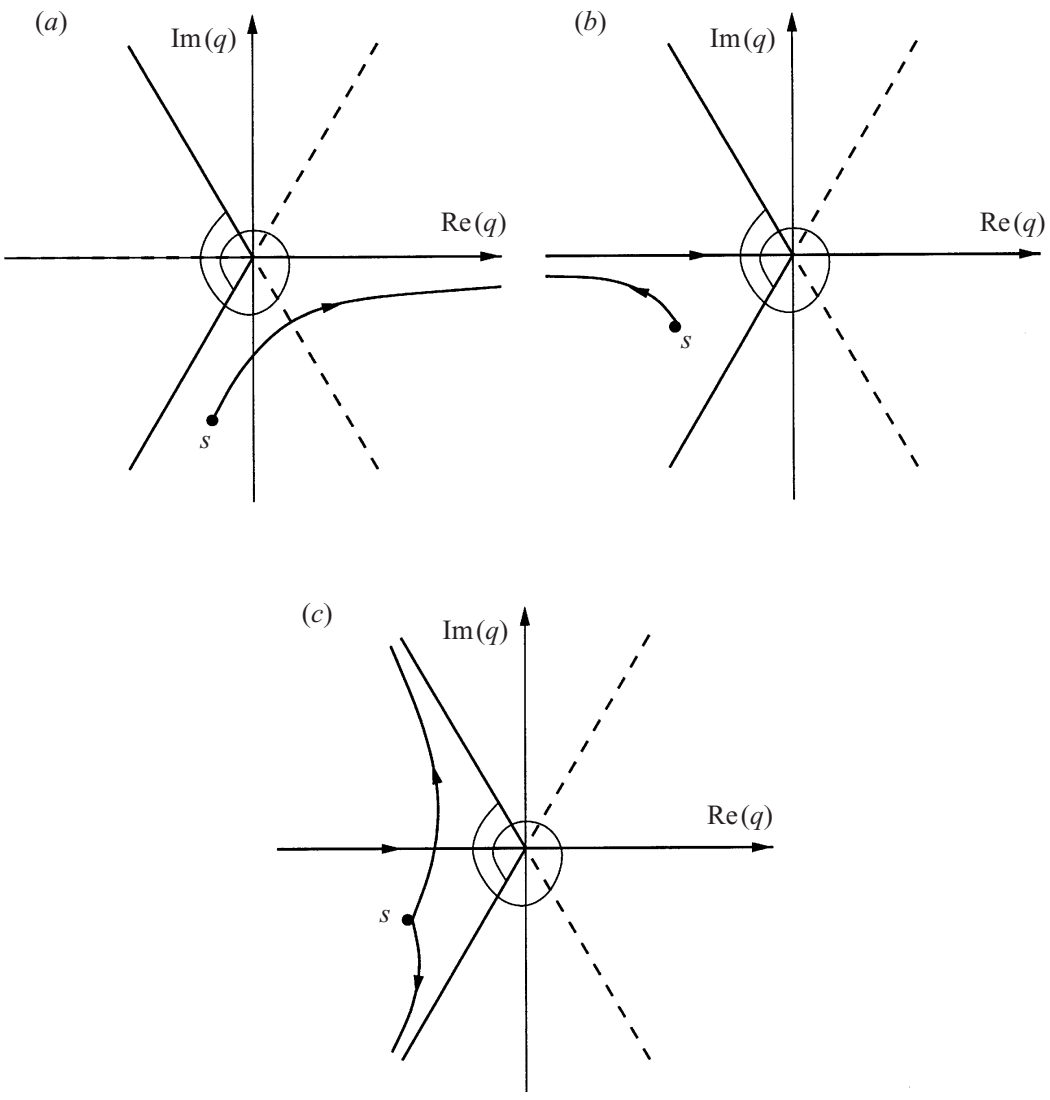

FIGURE 27. Steepest descent paths for (D 5).

as required. Now, for $\arg (s)<-2 \pi / 3$ the steepest descent path from the dominant WKB approximation to the Airy function tends to $s=+\infty \mathrm{e}^{-4 i \pi / 3}$ rather than $s=+\infty$. However, because of the Stokes phenomenon in the Airy function we must be careful not to deform the contour to this path, on which the other WKB approximation is present, which grows at infinity. We deform first to a contour from the endpoint to $-\infty$ plus an integration along the real axis, as shown in figure $27(b)$. Then we split the integration along the contour from the endpoint to $-\infty$ in to the two WKB approximations, and deform one contour to go to $s=+\infty \mathrm{e}^{-4 i \pi / 3}$ and one to go to $s=+\infty \mathrm{e}^{-2 \mathrm{i} \pi / 3}$, as shown in figure $27(c)$. Since neither path goes through the turning point the WKB approximations are valid. However, for the integration along the real axis we cannot replace the Airy function by its WKB approximation.

Thus we find that across the Stokes line we have two endpoint contributions, corresponding to the two WKB solutions, and the integral along the real axis, corresponding to the inviscid solution. This latter contribution is

$$
\bar{v} \sim-2 \pi^{1 / 2} s \int_{-\infty}^{\infty} \operatorname{Ai}(q) \mathrm{d} q=-2 \pi^{1 / 2} s .
$$

Hence, in $v$, the amount of inviscid solution turned on is

$$
-\frac{2^{1 / 6} \pi^{1 / 2} \mathrm{se}^{-5 \mathrm{i} \pi / 8}(\alpha R)^{5 / 12}}{(\lambda-1)^{5 / 12}} .
$$


Now, in the inner coordinates

$$
v_{2} \sim(2 \alpha R)^{-1 / 3}(\lambda-1)^{-1 / 6} s .
$$

Hence the Stokes multiplier is

$$
-\frac{2^{1 / 2} \pi^{1 / 2} \mathrm{e}^{-5 \mathrm{i} \pi / 8}(\alpha R)^{3 / 4}}{(\lambda-1)^{1 / 4}}
$$

so that

$$
b=-\frac{2^{1 / 2} \pi^{1 / 2} \mathrm{e}^{-5 \mathrm{i} \pi / 8}}{(\lambda-1)^{1 / 4}} .
$$

\section{Appendix E. Nonlinear oblique mode interactions}

Let us first note that the phases of the nonlinear terms in (3.23), (3.24) automatically satisfy the eikonal equation, so that the nonlinear terms are effectively forcing the amplitude equations (4.22), (4.23). We begin by determining the size of the optimally growing oblique-mode perturbation required to bring the nonlinear terms in (3.23), (3.24) into play if the initial conditions are such that the blow-up times for all wavenumbers are the same. Then the nonlinear terms also feed back onto modes with this blow-up time. During the first phase of the evolution the left-hand side of (3.23) is order $R^{1 / 3} v_{0}$ while the right-hand side is order $R v_{0}^{2}$, giving $v_{0}=O\left(R^{-2 / 3}\right)$. Likewise the left-hand side of (3.24) is order $v_{0}$, while the right-hand side is order $R^{2 / 3} v_{0}^{2}$.

During the second phase the left-hand sides of (3.23) and (3.24) are order $R^{2 / 3} v_{0}$, while the right-hand sides are order $R^{4 / 3} v_{0}^{2}$.

During the third phase the left-hand side of (3.24) is order $R^{1 / 3} v_{0}$ while the righthand side is order $R^{4 / 3} v_{0}^{2}$. Similarly the left-hand side of (3.23) is order $R^{1 / 3} v_{0}$ while the right-hand side is order $R^{5 / 3} v_{0}^{2}$. As expected, the third phase is the dominant one. However, even though (3.23) would seem to imply a nonlinear effect when $v_{0}=O\left(R^{-4 / 3}\right)$, in fact the forcing term in (3.23) is a function of $\eta$ only, and there is no forcing term in the $\eta$-equation with this scaling, so that $v$ simply grows transiently as $\eta$ decays. To obtain a true feedback requires the scaling $v_{0}=O\left(R^{-1}\right)$ so that the nonlinear terms in (3.24) come into play.

There is no boot-strapping in the method of transition described above - the initial transient growth simply gives the solution a kick start. The effect of the transient growth will be greater if the nonlinear terms can feed the large amplitude of two modes past their blow-up times back into another mode before its blow-up time. From (4.32), (4.33) we see that the blow-up time of the mode fed by the product of modes with streamwise wavenumbers $n_{1}$ and $n_{2}$ and blow-up times $\tilde{t}_{1}$ and $\tilde{t}_{2}$ is the weighted average

$$
\frac{n_{1} \tilde{t}_{1}+n_{2} \tilde{t}_{2}}{n_{1}+n_{2}} .
$$

If $n_{1}$ and $n_{2}$ have opposite sign then this time can be greater than both $\tilde{t}_{1}$ and $\tilde{t}_{2}$. Then the amplitude of the new mode generated by the nonlinear terms is order $R^{4 / 3} v_{0}^{2}$. Thus the cut-off for the new mode having larger amplitude than the original two modes is $v_{0}=O\left(R^{-4 / 3}\right)$. In order to generate a boot-strapping effect we need to feed this mode back into another with later blow-up time and so on. If this is possible ad infinitum (which is not immediately clear) then the threshold is $v_{0}=O\left(R^{-4 / 3}\right)$. Since $v_{0}$ varies on the lengthscale $R^{-1 / 3}$ this implies that the threshold norm is $O\left(R^{-1}\right)$.

Note that for the route to transition (ii) the size of the oblique-mode perturbation 
was $v_{0}=O\left(R^{-4 / 3}\right)$, so that the nonlinear terms in (3.23) and (3.24) are indeed negligible in that case.

\section{REFERENCES}

Baggett, J. \& Trefethen, L. 1997 Low-dimensional models of subcritical transition to turbulence. Phys. Fluids 9, 1043-1053.

Benney, D. J. 1961 A nonlinear theory for oscillations in a parallel flow. J. Fluid Mech. 10, 209-236.

BENNEY, D. J. 1964 Finite amplitude effects in an unstable laminar boundary layer. Phys. Fluids 7, 319-326.

Benney, D. J. \& Chow, K. 1985 An alternative approach to nonlinear instabilities in hydrodynamics. Stud. Appl. Maths 73, 261-267.

Benney, D. J. \& Gustavsson, L. H. 1981 A new mechanism for linear and nonlinear hydrodynamic instability. Stud. Appl. Maths 64, 185-209.

BenNey, D. J. \& Lin, C. C. 1960 On the secondary motion induced by oscillations in a shear flow. Phys. Fluids 3, 656-657.

Boberg, L. \& Brosa, U. 1988 Onset of turbulence in a pipe. Z. Naturforsch. A 43, 679-726.

Butler, K. M. \& FARrell, B. F. 1992 Three-dimensional optimal perturbations in viscous shear flow. Phys. Fluids A 4, 1637-1650.

Chapman, S. J., King, J. R. \& Adams, K. L. 1998 Exponential asymptotics and Stokes lines in nonlinear ordinary differential equations. Proc. Soc. Lond. A 454, 2733-2755.

Chapman, S. J., Lawry, J. M. H., Ockendon, J. R. \& Tew, R. H. 1999 On the theory of complex rays. SIAM Rev. 41, 417-509.

Cherhabili, A. \& Ehrenstein, U. 1997 Finite-amplitude equilibrium states in plane Couette flow. J. Fluid Mech. 342, 159-177.

Corcos, G. M. \& Sellars, J. R. 1959 On the stability of fully developed flow in a pipe. J. Fluid Mech. 5, 97-112.

Cowley, S. J. \& Smith, F. T. 1985 On the stability of Poiseuille-Couette flow: a bifurcation from infinity. J. Fluid Mech. 156, 83-100.

Dahlhuis, A. B. O., Chapman, S. J., King, J. R., Ockendon, J. R. \& Tew, R. H. 1995 Stokes phenomenon and matched asymptotic expansions. SIAM J. Appl. Maths 55, 1469-1483.

DaveY, A. 1973 On the stability of plane Couette flow to infinitesimal disturbances. J. Fluid Mech. 57, 369-380.

Davey, A. \& Drazin, P. G. 1969 The stability of Poiseuille flow in a pipe. J. Fluid Mech. 36, 209-218.

Davey, A. \& Nguyen, H. P. F. 1971 Finite-amplitude stability of pipe flow. J. Fluid Mech. 45, 701-720.

Drazin, P. G. \& ReID, W. H. 1981 Hydrodynamic stability. Cambridge University Press.

Ehrenstein, U. \& KосH, W. 1991 Three-dimensional wave-like equilibrium states in plane Poiseuille flow. J. Fluid Mech. 228, 111-148.

FArrell, B. F. 1988 Optimal excitation of perturbations in viscous shear flow. Phys. Fluids 31, 2093-2102.

Gill, A. E. 1965 On the behaviour of small disturbances to Poiseuille flow in a circular pipe. J. Fluid Mech. 21, 145-172.

Gustavsson, L. H. 1986 Excitation of direct resonances in plane Poiseuille flow. Stud. Appl. Maths 75, 227-248.

Gustavsson, L. H. 1991 Energy growth of three-dimensional disturbances in plane Poiseuille flow. J. Fluid Mech. 224, 241-260.

Gustavsson, L. H. \& Hultgren, L. S. 1980 A resonance mechanism in plane Couette flow. J. Fluid Mech. 98, 149-159.

Henningson, D. S. 1991 An eigenfunction expansion of localized disturbances. In Advances in Turbulence 3 (ed. A. V. Johansson \& P. H. Alfredsson), pp. 162-169. Springer.

Henningson, D. S., Lundbladh, A. \& Johansson, A. V. 1993 A mechanism for bypass transition from localized disturbances in wall-bounded shear flows. J. Fluid Mech. 250, 169-207.

JANG, P. S., BENNEY, D. J. \& Gran, R. L. 1986 On the origin of streamwise vortices in a turbulent boundary layer. J. Fluid Mech. 169, 109-123. 
Kreiss, G., Lundbladh, A. \& Henningson, D. S. 1994 Bounds for threshold amplitudes in subcritical shear flows. J. Fluid Mech. 270, 175-198.

Lundbladh, A., Henningson, D. S. \& Reddy, S. C. 1994 Threshold amplitudes for transition in channel flows. In Transition, Turbulence and Combustion (ed. M. Hussaini, T. Gatski \& T. Jackson), pp. 309-318. Kluwer.

Morawetz, C. S. 1952 The eigenvalues of some stability problems involving viscosity. J. Rat. Mech. Anal. 1, 579-603.

NAGATA, M. 1990 Three-dimensional finite-amplitude solutions in plane Couette flow: bifurcation from infinity. J. Fluid Mech. 217, 519-527.

NG, B. S. \& REID, W. H. 1999 Asymptotic analysis of a fourth-order turning-point problem in hydrodynamic stability. Q. J. Mech. Appl. Maths 53, 27-42.

ORR, W. M. 1907 The stability or instability of the steady motions of a perfect liquid and of a viscous liquid. Proc. R. Irish Acad. A 27, 9-68 and 69-138.

OrszaG, S. A. 1971 Accurate solution of the Orr-Sommerfeld stability equation. J. Fluid Mech. 50, 689.

Orszag, S. A. \& Kells, L. C. 1980 Transition to turbulence in plane Poiseuille and plane Couette flow. J. Fluid Mech. 96, 159-205.

Orszag, S. A. \& Patera, A. T. 1983 Secondary instability of wall-bounded shear flows. J. Fluid Mech. 128, 347-385.

Reddy, S. C. \& Henningson, D. S. 1993 Energy growth in viscous channel flows. J. Fluid Mech. 252, 209-238.

Reddy, S. C., Schmid, P. J., Baggett, J. S. \& Henningson, D. S. 1998 On stability of streamwise streaks and transition thresholds in plane channel flows. J. Fluid Mech. 365, 269-303.

Reddy, S. C., Schmid, P. J. \& Henningson, D. S. 1993 Pseudospectra of the Orr-Sommerfeld operator. SIAM J. Appl. Maths 53, 15-47.

Reynolds, W. C. \& Potter, M. C. 1967 Finite-amplitude instability of parallel shear flows. J. Fluid Mech. 27, 465-492.

Romanov, V. A. 1973 Stability of plane-parallel Couette flow. Funct. Anal. Applics. 7, 137-146.

Shantini, R. 1989 Degeneracies of the temporal Orr-Sommerfeld eigenmodes in plane Poiseuille flow. J. Fluid Mech. 201, 13-34.

SQuire, H. B. 1933 On the stability for three-dimensional disturbances of viscous fluid between parallel walls. Proc. R. Soc. Lond. A 142, 621-628.

StUART, J. T. 1960 On the non-linear mechanics of wave disturbances in stable and unstable parallel flows. Part 1. The basic behaviour in plane poiseuille flow. J. Fluid Mech. 9, 353-370.

Thomson, W. 1887 (Lord Kelvin) Stability of fluid motion - rectilineal motion of viscous fluid between two parallel planes. Phil. Mag. 24, 188-196.

Trefethen, L. N., Trefethen, A. E., Reddy, S. C. \& Driscoll, T. A. 1993 Hydrodynamic stability without eigenvalues. Science 261, 578-584.

WATSON, J. 1960 On the non-linear mechanics of wave disturbances in stable and unstable parallel flows. Part 1. The development of a solution for plane Poiseuille flow and plane Couette flow. J. Fluid Mech. 9, 371-389. 